Issued by Sandia National Laboratories, operated for the United States Department of Energy by Sandin Compration.

NoTicE: This report was prepared as an account of work sponsored by an agency of the United States Government. Neither the United States Government nor any agency thereof, nor any of their employees, nor any of their contractors, subeontractors, or their employees, makes any warranty, express or implied ox assumes any legal labllity or responstbility for the accuracy, completeness, or pseruness of any information, apparatus, pwned w red

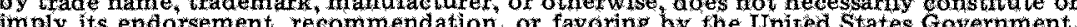
any arency thereof or any of theit contractors or subcom expressed herein do Government, any agency thereof or any of their contractors or subcontractors.

Printed in the United States of America Avallable from

National Technical Information Service

U. S. Department of Commerce

5285 Port Royal Road

Springfreld, VA 22161

NTIS price codes

Printed copy: $\$ 7.00$

Mictofiche copy: A01

$* * 2^{*} *$ 


\section{DISCLAIMER}

This report was prepared as an account of work sponsored by an agency of the United States Government. Neither the United States Government nor any agency Thereof, nor any of their employees, makes any warranty, express or implied, or assumes any legal liability or responsibility for the accuracy, completeness, or usefulness of any information, apparatus, product, or process disclosed, or represents that its use would not infringe privately owned rights. Reference herein to any specific commercial product, process, or service by trade name, trademark, manufacturer, or otherwise does not necessarily constitute or imply its endorsement, recommendation, or favoring by the United States Government or any agency thereof. The views and opinions of authors expressed herein do not necessarily state or reflect those of the United States Government or any agency thereof. 


\section{DISCLAIMER}

Portions of this document may be illegible in electronic image products. Images are produced from the best available original document. 


\title{
Development and Testing of FIDELE (A Computer Code for Finite-Difference Solution to Harmonic Magnetic-Dipole Excitation of an Azimuthally Symmetric Horizontally and Radially Layered Earth)
}

\author{
Charles N. Vittitoe \\ Theoretical Division 4231 \\ Sandia National Laboratories \\ Albuquerque, NM 87185
}

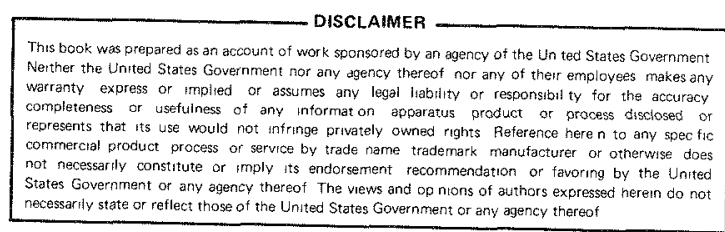

States Government or any agency thereof The views and op nions of authors expressed heren do not

\begin{abstract}
The FORTRAN IV computer code FIDELE simulates the high-frequency electrical logging of a well in which induction and receiving coils are mounted in an instrument sonde immersed in a drilling fluid. The fluid invades layers of surrounding rock in an azimuthally symmetric pattern, superimposing radial layering upon the horizontally layered earth. Maxwell's equations are reduced to a second-order elliptic differential equation for the azimuthal electric-field intensity. The equation is solved at each spatial position where the complex dielectric constant, magnetic permeability, and electrical conductivity have been assigned. Receiver response is given as the complex open-circuit voltage on receiver coils. The logging operation is simulated by a succession of such solutions as the sonde traverses the borehole. Test problems verify consistency with available results for simple geometries. The code's main advantage is its treatment of a two-dimensional earth; its chief disadvantage is the large computer time required for typical problems. Possible code improvements are noted. Use of the computer code is outlined, and tests of most code features are presented.
\end{abstract}




\section{Acknowledgments}

I thank P.C. Lysne for proposing a series of test problems and G. A. Seely and D. L. Hicks for discussions of numerical methods. The work also benefited from the encouragement and support of J. H. Renken and P. C. Lysne. The work was supported by the Department of Energy's Western Gas Sands Project administered by the Bartlesville Energy Technology Center. The Technical Project Officer was Dr. James B. Jennings. 


\section{Contents}

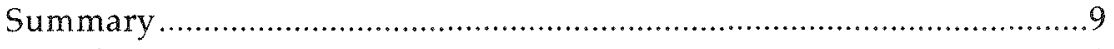

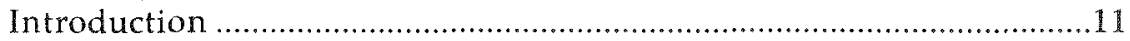

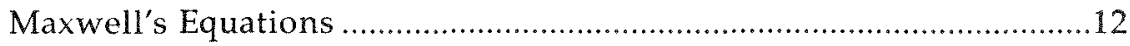

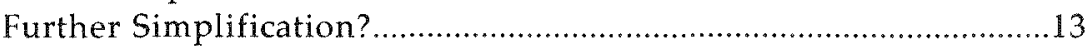

Harmonic Analysis .............................................................................13

Harmonic Magnetic Dipole in a Homogeneous Isotropic Medium ....13

Formulation of Finite-Difference Equations in a

Heterogeneous Medium .................................................................14

Successive Overrelaxation (SOR) Solution--Limitations ..................15

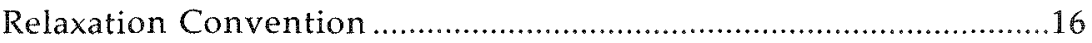

Boundary Conditions .................................................................16

Axial Boundary Condition ..........................................................17

Alternative Form for Outer Boundary Conditions.......................18

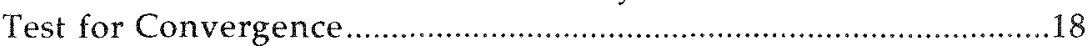

Nonuniform Radial Mesh ..........................................................19

Test Problem A: Effect of Neumann Boundary Conditions

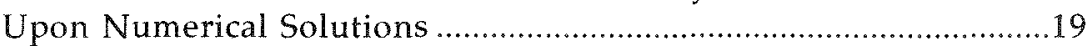

Test Problem B: Earth With One Vertical Interface .............................20

Lysne Test Problems and Comparison With Some Available

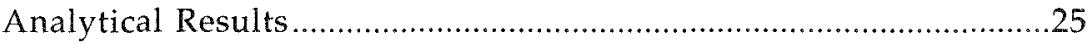

Test Problem C: Oil-Drilling Fluid, Frequency $1 \mathrm{MHz} \ldots \ldots \ldots \ldots \ldots \ldots \ldots \ldots . .25$

Possible Changes in IN (Input) Subroutine .......................................25

Possible Changes in EP (Electrical Parameter) Subroutine ..............26

Possible Changes in Main Program FIDELE...................................26

Possible Control Card Changes............................................................26

Test Problem D: Saltwater Drilling Fluid, Frequency $1 \mathrm{MHz}$.............31

Test Problem E: Saltwater Drilling Fluid, Frequency $30 \mathrm{MHz}$...........37

Test Problem F: Oil-Drilling Fluid, Frequency $30 \mathrm{MHz}$..................41

Comparison With Analytical Results ................................................42

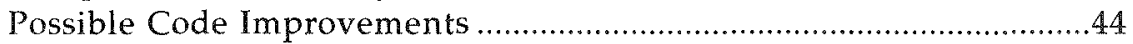

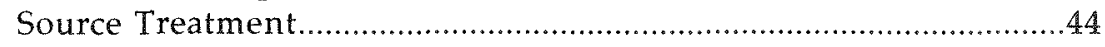

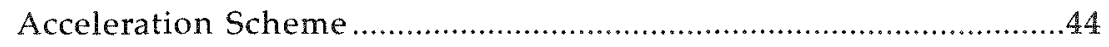

Mesh Size Alteration and Initial Guess Improvement ......................44

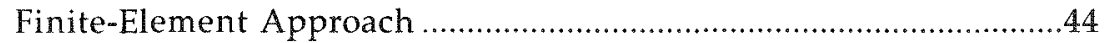

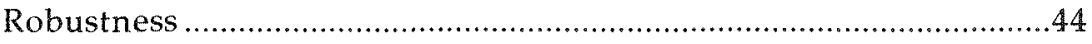

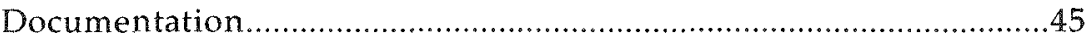

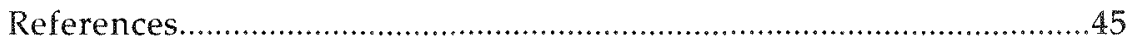

APPENDIX A - An Acceleration Scheme ….........................................47

APPENDIX B - Sensitivity to Actual Value of $f$ at Outer Boundaries ...............................................................49

APPENDIX C - Test Case for Nonuniform Radial Mesh ..................51

APPENDIX D - Test Case for Mesh-Size Alteration After

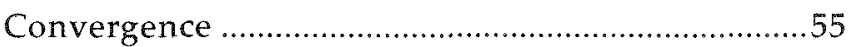

APPENDIX E - Use of FIDELE Computer Code ……........................57

APPENDIX F - Use of the DIPPL Computing Code for Plotting Results of FIDELE....................................................59

APPENDIX G - Computer Time Requirements .................................61 


\section{Illustrations}

\section{Figure}

1 Simulation Geometry ................................................................... 11

2 The Finite-Difference Grid ......................................................... 14

3 Geometry for MK Problem ........................................................ 20

4 Variation of Convergence Parameter S1 With Iteration Number for Test Problem B ......................................................................... 21

5 Apparent Conductivity at Two Positions in the Mesh of Test Problem B ................................................................................. 22

6 Apparent Conductivity vs Transmitter to Receiver-Coil Spacing (L) for Test Problem B .............................................................. 22

7 Real (S) Distribution as a Function of $\mathrm{r}[=(\mathrm{I}-1) \mathrm{DT}]$ and $z[=(J-1) D Z]$ Illustrating an Error in the Source Region for an Early Calculation of Test Problem B...................................... 22

8 Soil-Layering Geometry for Test Problem B................................ 23

9 Real S( $z, r)$ Distribution for Test Problem B ................................. 23

10 Real Part of Voltage in a Receiver Coil at Axial Distance L From the Dipole Source in Test Problem B........................................... 23

11 Imaginary S(z,r) Distribution for Test Problem B....................... 24

12 Imaginary Part of Voltage in a Receiver Coil at Axial Distance L From the Dipole Source in Test Problem B ................................. 24

13 Phase Angle of the Complex Voltage in a Receiver Coil at Axial Distance L From the Dipole Source in Test Problem B .............. 24

14 Variation of Convergence Parameter S1 With Iteration Number for Test Problem C....................................................................... 27

15 Real Part of the Complex Voltage in Receiver Coils at $L=0.62$ and $0.92 \mathrm{~m}$ for Test Problem C ................................................. 27

16 Soil-Layering Geometry for Test Problem C …........................... 27

17 Real S(z,r) Distribution for Test Problem C................................ 27

18 Real Part of Voltage in a Receiver Coil at Axial Distance L From the Dipole Source in Test Problem C .......................................... 28

19 Imaginary Part of Voltage in a Receiver Coil at Axial Distance L From the Dipole Source in Test Problem C............................... 28

20 Phase Angle of the Complex Voltage in a Receiver Coil at Axial Distance L From the Dipole Source in Test Problem C............. 29

21 Variation of Convergence Parameter S1 With Iteration Number for Modified Test Problem C .......................................................... 29

22 Real Part of the Complex Voltage in Receiver Coils at $\mathrm{L}=0.61$ and $0.91 \mathrm{~m}$ for Modified Test Problem C...................................29

23 Real S(z,r) Distribution for Modified Test Problem C ................. 30

24 Soil-Layering Geometry for Modified Test Problem C............... 30

25 Real Part of Voltage in a Receiver Coil at Axial Distance L From the Dipole Source in Modified Test Problem C......................... 30

26 Imaginary Part of Voltage in a Receiver Coil at Axial Distance L From the Dipole Source in Modified Test Problem C ................ 31

27 Phase Angle of the Complex Voltage in a Receiver Coil at Axial Distance L From the Dipole Source in Modified Test Problem C 


\section{Illustrations (cont)}

\section{Figure}

28 Variation of Convergence Parameter S1 With Iteration Number for Test Problem D....

29 Real Part of the Complex Voltage in Receiver Coils at $\mathrm{L}=0.62$ and $0.92 \mathrm{~m}$

30 Real S(z,r) Distribution for Test Problem D

31 Real Part of the Complex Voltage in a Receiver Coil at Axial Distance L From the Dipole Source in Test Problem D............. 33

32 Imaginary Part of the Complex Voltage in a Receiver Coil at Axial Distance L From the Dipole Source in Test Problem D... 33

33 Phase Angle of the Complex Voltage in a Receiver Coil at Axial Distance L From the Dipole Source in Test Problem D.

34 Variation of Convergence Parameter S1 With Iteration Number for Modified Test Problem D

35 Real Part of the Complex Voltage in Receiver Coils at $\mathrm{L}=0.61$ and $0.91 \mathrm{~m}$ for Modified Test Problem D ............................ 35

36 Imaginary Part of the Complex Voltage in Receiver Coils at $\mathrm{L}=0.61$ and $0.91 \mathrm{~m}$ for Modified Test Problem $\mathrm{D}$................... 35

37 Soil-Layering Geometry for Modified Test Problem D............. 35

38 Real S(z,r) Distribution for Modified Test Problem D ............... 35

39 Real Part of Voltage in a Receiver Coil at Axial Distance L From the Dipole Source in Modified Test Problem D ........................ 36

40 Imaginary Part of Voltage in a Receiver Coil at Axial Distance L From the Dipole Source in Modified Test Problem D ............... 36

41 Phase Angle of the Complex Voltage in a Receiver Coil at Axial Distance L From the Dipole Source in Modified Test Problem D.

42 Variation of Convergence Parameter S1 With Iteration Number for Test Problem E

43 Convergence of the Real and Imaginary Parts of the Complex Voltage on a Receiver Coil for Test Problem E......................... 37

44 Real S $(z, r)$ Distribution for Test Problem E ................................ 38

45 Real Part of the Complex Voltage in a Receiver Coil at Axial Distance L From the Dipole Source for Test Problem E........... 38

46 Imaginary Part of the Complex Voltage in a Receiver Coil at Axial Distance L From the Dipole Source for Test Problem E.

47 Phase Angle of the Complex Voltage in a Receiver Coil at Axial Distance L From the Dipole Source for Test Problem E............ 39

48 Soil-Layering Geometry for Modified Test Problem E .............. 40

49 Real S(z,r) Distribution for Modified Test Problem E............... 40

50 Real and Imaginary Parts of the Complex Voltage in a Receiver Coil at Axial Distance L From the Dipole Source in Modified Test Problem E.

51 Phase Angle of the Complex Voltage in a Receiver Coil at Axial Distance L From the Dipole Source in Modified Test Problem E. 


\section{Illustrations (cont)}

\section{Figure}

52 Real Part of the Complex Voltage in a Receiver Coil at $\mathrm{L}=$ $0.18 \mathrm{~m}$ for Test Problem E and Modified Test Problem E.......... 41

53 Variation of Convergence Parameter S1 With Iteration Number for Test Problem F

54 Real Part of the Complex Voltage in a Receiver Coil at $L=0.92 \mathrm{~m}$ for Test Problem F.................................................. 42

55 Real S(z,r) Distribution for Test Problem F ................................ 42

56 Real Part of the Complex Voltage in a Receiver Coil at Axial Distance L From the Dipole Source for Test Problem F............ 43

57 Imaginary Part of the Complex Voltage in a Receiver Coil at Axial Distance L From the Dipole Source for Test Problem F

58 Phase Angle of the Complex Voltage in a Receiver Coil at Axial Distance L From the Dipole Source for Test Problem $F$

\section{Tables}

\section{Table}

1 Illustration of Convergence for Magnetic Dipole in Uniform

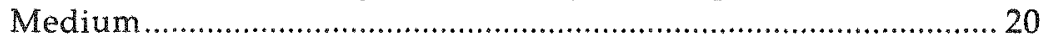

2 Convergence Near Boundary for Chosen Problem ..................... 20

3 Apparent Conductivity in Test Problem B................................... 21

4 Check of $\mathrm{r}^{2}$ Variation for Real Part of the Complex Coil Voltage at $\mathrm{L}=0.76 \mathrm{~m}$ 


\section{Summary}

This report describes the development and testing of FIDELE, a FORTRAN IV computer code yielding the FInite-difference solution for harmonic magnetic-Dipole Excitation of a radially and horizontally Layered Earth. Such geometries and excitations approximate those produced by a magnetic-induction logging tool operated at high frequencies in reservoir-analysis operations. The tool case, the drill hole, and the drilling-fluid invasion into surrounding rock all furnish radial layers; the geology of the region and the tool case furnish horizontal layers. Signals received by coils mounted in the same tool are influenced by the electrical parameters of the surrounding rock. These parameters are important because of correlations with reservoir properties such as porosity, water saturation, and (perhaps) permeability. The problem has been solved to enable users to study the sensitivity of received signals to variations in the complex dielectric constant, conductivity, and magnetic permeability of the surrounding rock.

The main purpose of the code is to predict receiver response to harmonic magnetic-dipole excitation of a layered earth with known electrical parameters. The receiver is modeled to respond to the opencircuit voltage across the receiver coil. The source is represented as a point magnetic dipole embedded in a layered earth.

Maxwell's equations in a cylindrically symmetric geometry with harmonic excitation result in a second-order elliptic partial-differential equation for the azimuthal electric-field intensity. A change of variables reduces the order of the solution singularity at the source position. Approximating the resulting differential equation by finite differences results in a system of equations solved by successive overrelaxation techniques.

Boundary conditions impose restrictions upon the solution. Inside the region surrounding the source, excitation is assumed to be that of a magnetic dipole in a homogeneous, isotropic medium with parameters representative of the sonde material. Here the source is very close and is assumed to dominate the source region. The azimuthal symmetry furnishes a convenient Neumann boundary condition along the symmetry axis. The remaining outer boundary conditions are the most difficult to treat accurately.

A Neumann boundary condition based upon the homogeneous-medium solution is formulated for the outer boundaries. It uses only local values of electricfield intensity and is reasonable when all virtual sources (created by layering effects) are close to the source position. Signal sensitivity to variations of these boundary conditions is examined and found to be a minor perturbation in several tests.

A series of test problems is analyzed to indicate accuracy of the code and its limitations. The code is verified for a homogeneous, isotropic medium where analytic solution is available. Solution for a problem with one vertical interface and excitation at $20 \mathrm{kHz}$ is verified by published data; i.e., except for receiver positions that are more than $80 \%$ of the distance from the source to the outer boundary. This suggests that the outer boundary condition can be improved. Solutions for test problems that model a geometry with two vertical interfaces, with realistic parameter choices, and with excitation at $1 \mathrm{MHz}$ and $30 \mathrm{MHz}$, are consistent with data calculated elsewhere. These test problems illustrate the code's major handicap-the computer time consumed. On the CDC-7600 computer at Sandia National Laboratories in Albuquerque, the FIDELE code requires $2.5 \mathrm{~s}$ per iteration and several thousand iterations for solution.

Possible code improvements include generalization of the source being modeled, improved implementation of acceleration schemes, better initialization after mesh-size alteration to expand the domain of solution, increased robustness, and application of a finite-element approach rather than the finitedifference technique described. Most of these improvements are directed toward increasing speed of solution.

Appendices describe tests of several code capabilities: acceleration schemes, use of a nonuniform radial mesh, and mesh-size alteration after convergence to expand the domain of solution. Other appendices furnish directions for using FIDELE with new problems and for using DIPPL to give convenient graphical output of FIDELE results.

This report is written for code users and logging analysts. We assume that, although neither group is expert in electromagnetic theory or in the numerical approaches taken, some in each group are interested in understanding the work. Thus some readers will find portions too detailed; others, like an analyst desiring to improve a portion of the code, might benefit from even greater explanation. Success is attained if all find pertinent sections to inform and to enjoy. 
-

- 


\section{Development and Testing of FIDELE (A Computer Code for Finite-Difference Solution to Harmonic Magnetic-Dipole Excitation of an Azimuthally Symmetric Horizontally and Radially Layered Earth)}

\section{Introduction}

Work on a magnetic-dipole logging probe of boreholes began at Sandia National Laboratories as a possible aid in evaluating low-permeability tightgas-sand formations. If natural gas is abundant in a formation, the critical parameter is permeability; i.e., the ease of gas flow through the material. The electrical properties of the formation itself should be correlated with this permeability. Imagine the formation. as composed of grains with some space between them. As grain size is reduced, the grains are more tightly packed--and formation resistivity, effective dielectric constant (capacitance) between two electrodes in the formation, and foundation permeability are all reduced. Complicating factors such as groundwater and pore-shape distributions can alter such direct relations, but a correlation is still expected.

The layered earth may be interrogated by a series of frequencies. High frequencies allow a response that is completely determined by nearby materials such as the drilling fluid, its invasion zone, and perhaps the nearest horizontal interface. Once these nearby materials are identified, lower frequencies may enable queries at distances farther away.

Our earth is layered in a variety of ways. In some regions the geological structure gives horizontal layering. When such layers are inclined, the present treatment serves as an azimuthally symmetric approximation. Radial layers are formed by the sonde, by the boreholes, and by the drilling fluid and its invasion into the surrounding structure.

The most useful computer code for simulating an induction probe would accept source terms and received signals (and their uncertainties) as input quantities. Such a code would yield complex quantities (and corresponding uncertainties) for the effective dielectric constant, the magnetic permeability, and the permittivity of the surrounding material as a function of spatial coordinate and of source frequency. Further analysis should then yield the twodimensional distribution of earth porosity, water saturation, and permeability. This inverse process is not addressed here: it is much more difficult, requiring electrical parameter and geologic property correlation data not now available; it is also not expected to be unique, at least for one probe frequency and for completely arbitrary layering.

The computer code FIDELE finds the electric-field intensity at spatial positions surrounding a borehole harmonically excited by a magnetic dipole. The electrical properties of the earth are assumed known and axially symmetric about the borehole axis. The source is mounted inside a sonde case and is lowered into the borehole. Problem geometry is indicated in Figure 1. The receiver (or series of receivers) is also mounted in the sonde and treated as a coil oriented

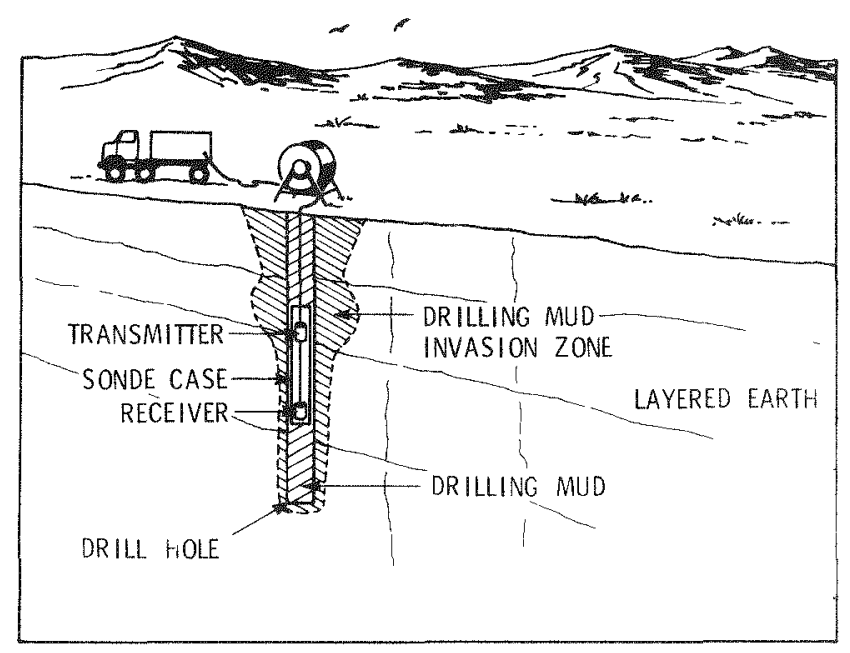

Figure 1. Simulation Geometry 
along the axis to detect the open-circuit voltage produced by the azimuthal electric-field intensity. The source is assumed to be a point magnetic dipole at a given frequency.

One possible use of FIDELE is to generate a series of cross-plots for dielectric-constant log interpretation. For example, sonde design could fix dielectric constant $\left(\epsilon / \epsilon_{\mathrm{o}}\right)$, conductivity $(\sigma)$, and dimensions for a reasonable source region that includes the magnetic dipole. Receiver positions are also fixed by sonde design. Borehole radius and drilling-fluid parameters can also be chosen to model a particular drilling scenario. Results of other logging probes can be used to model positions where horizontal interfaces occur and to estimate the conductivity of each horizontal layer. Geometrical variables remaining are the dielectric constant of the surrounding saturated rock, the radius of the invasion zone (as a function of horizontal layering), and the electrical parameters for the rock outside the invasion zone. It may be possible to select frequencies high enough to probe only as far as the invasion zone; then the radius of the invasion zone and the rock outside the invasion zone do not influence the calculations. A matrix of $\epsilon / \epsilon_{o}$ values can be chosen for FIDELE and the corresponding receiver response(s) tabulated. This procedure allows estimation of the $\epsilon / \epsilon_{\mathrm{o}}$ of the saturated invasion zone for each horizontal layer. Computer runs for several $\sigma$ values may also allow improvement of the estimates for conductivity in the saturated invasion zone (perhaps by checking for consistency between signal amplitude and phase for several receivers).

After invasion zone parameters are specified, lower-frequency excitation allows deeper probing. Radii of saturated zones vary with horizontal layering. The electrical properties of the transition zones and of the virgin rock are yet unknown. Even the invasion-zone electrical parameters are uncertain because of frequency variation. Data inversion in this geometry is expected to be difficult and may not be unique.

\section{Maxwell's Equations}

The electromagnetic fields generated by the magnetic dipole satisfy Maxwell's equations at all points in space. In nationalized mks units

$$
\begin{aligned}
& \nabla \times \underline{E}=-\frac{\partial B}{\partial t}, \\
& \nabla \times \underline{H}=I+\frac{\partial D}{\partial t} .
\end{aligned}
$$

The earth is assumed to have complex local values of permittivity $(\epsilon)$, magnetic permeability $(\mu)$, and conductivity $(\sigma)$ that are scalar functions of position. $B=$ $\mu \mathrm{H}, \mathrm{D}=\epsilon \mathrm{E}$, and $\mathrm{I}=\sigma \mathrm{E}$. Substitution of these consti. tutive relations into Maxwell's equations and use of cylindrical coordinates $(r, \theta, z)$ yields the following components of Maxwell's equations:

$r$ components

$$
\begin{aligned}
& \frac{1}{\mathrm{r}} \frac{\partial \mathrm{E}_{\mathrm{z}}}{\partial \theta}-\frac{\partial \mathrm{E}_{\theta}}{\partial \mathrm{z}}=-\frac{\partial \mathrm{B}_{\mathrm{r}}}{\partial \mathrm{t}} \\
& \frac{1}{\mathrm{r}} \frac{\partial \mathrm{H}_{\mathrm{z}}}{\partial \theta}-\frac{\partial \mathrm{H}_{\theta}}{\partial \mathrm{z}}=\sigma \mathrm{E}_{\mathrm{r}}+\epsilon \frac{\partial \mathrm{E}_{\mathrm{r}}}{\partial \mathrm{t}}
\end{aligned}
$$

$\theta$ components

$$
\begin{aligned}
& \frac{\partial \mathrm{E}_{\mathrm{r}}}{\partial \mathrm{z}}-\frac{\partial \mathrm{E}_{\mathrm{z}}}{\partial \mathrm{r}}=-\mu \frac{\partial \mathrm{H}_{\theta}}{\partial \mathrm{t}} \\
& \frac{\partial \mathrm{H}_{\mathrm{r}}}{\partial \mathrm{z}}-\frac{\partial \mathrm{H}_{\mathrm{z}}}{\partial \mathrm{r}}=\sigma \mathrm{E}_{\theta}+\epsilon \frac{\partial \mathrm{E}_{\theta}}{\partial \mathrm{t}}
\end{aligned}
$$

$\mathrm{z}$ components

$$
\begin{aligned}
& \frac{1}{r}\left\{\frac{\partial}{\partial r}\left(\mathrm{rE}_{\theta}\right)-\frac{\partial \mathrm{E}_{\mathrm{r}}}{\partial \theta}\right\}=-\mu \frac{\partial \mathrm{H}_{z}}{\partial \mathrm{t}} \\
& \frac{1}{r}\left\{\frac{\partial}{\partial \mathrm{r}}\left(\mathrm{rH}_{\theta}\right)-\frac{\partial \mathrm{H}_{\mathrm{r}}}{\partial \theta}\right\}=\sigma \mathrm{E}_{z}+\epsilon \frac{\partial \mathrm{E}_{z}}{\partial \mathrm{t}} .
\end{aligned}
$$

With the cylindrical symmetry, all $\partial / \partial \theta=0$ and the equations can be grouped into two independent sets--those for $\left(\mathrm{E}_{\mathrm{r}^{\prime}}, \mathrm{E}_{\mathrm{z}^{\prime}}, \mathrm{H}_{\theta}\right)$ and those for $\left(\mathrm{H}_{\mathrm{r}^{\prime}} \mathrm{H}_{z^{\prime}}, \mathrm{E}_{\theta}\right)$. A magnetic-dipole source produces no fields $\mathrm{E}_{\mathrm{r}^{\prime}} \mathrm{E}_{\mathrm{z}^{\prime}}$ or $\mathrm{H}_{\theta}$; hence, with cylindrical symmetry, these compoents remain zero. The remaining equations for the transverse-electric fields may be written

$$
\begin{aligned}
& \frac{\partial \mathrm{B}_{\mathrm{r}}}{\partial \mathrm{z}}-\frac{\partial \mathrm{B}_{\mathrm{z}}}{\partial \mathrm{r}}=\mu \sigma \mathrm{E}_{\theta}+\mu \epsilon \frac{\partial \mathrm{E}_{\theta}}{\partial \mathrm{t}}, \\
& \frac{\partial \mathrm{E}_{\theta}}{\partial \mathrm{z}}=\frac{\partial \mathrm{B}_{\mathrm{r}}}{\partial \mathrm{t}}, \\
& \frac{\mathrm{E}_{\theta}}{\mathrm{r}}+\frac{\partial \mathrm{E}_{\theta}}{\partial \mathrm{r}}=-\frac{\partial \mathrm{B}_{z}}{\partial \mathrm{t}} .
\end{aligned}
$$

If the conductivity is zero, interchange of variables in the two independent sets above and interchange of $\mu$ 
and $\epsilon$ make the equations for the two sets identical.* In this application, however, conductivity must be included.

The electromagnetic fields obey Eqs (9) through (11) at every point in the earth. In addition, these electromagnetic fields satisfy boundary conditions at interfaces where local ground parameters change. The conditions required here are that the normal component of $B$ and the tangential component of $E$ be continuous across each interface. At large distances (R) from the source, beyond the attenuating materials the electromagnetic fields are outgoing waves with the variation $e=f(t-R / S) / R$ where $S$ is the velocity of light in the earth and $f$ is some causal function such that $\mathrm{f}(\mathrm{t}-\mathrm{R} / \mathrm{S})=0$ for $\mathrm{t}<\mathrm{R} / \mathrm{S}$. The system of simultaneous partial-differential Eqs (9) through (11) can be solved subject to the boundary conditions to yield the description of the electromagnetic fields generated in the earth.

\section{Further Simplification?}

It would be helpful if Eqs (9) through (11) could be further simplified. For example, at sufficiently low frequencies the $\partial / \partial t$ terms may be neglected and a quasistatic solution obtained. At high frequencies the conduction current $\left(\sigma \mathrm{E}_{\theta}\right)$ may be neglected. The critical parameter is $\omega \epsilon / \sigma$. Our goal is to allow the analysis to apply to a wide range of geologic materials. Dry granite, for example, has $\epsilon \approx 10 \epsilon_{\mathrm{o}}$ over the frequency range $10^{2}$ to $10^{6} \mathrm{~Hz}$, while its conductivity varies from $7 \times 10^{-7}$ siemens $\left(\mathrm{mho} / \mathrm{m}\right.$ ) to $8 \times 10^{-5} \mathrm{mho} / \mathrm{m}$. Hence its $\omega \epsilon / \sigma$ can vary from 0.08 to 6 . Because geological materials with higher conductivity tend to have larger dielectric constants, especially at low frequencies, both the conduction current and displacement current are included so as to maintain generality in our results.

Another possiblity is the neglect of some spatial derivatives with respect to time derivatives. This would severely limit the allowable interfaces between geologic materials and would probably limit the frequency range through the connection between skin depth and frequency. Rather than further simplification, a greater need appears to be increased generality to include frequency-dependent electrical parameters $(\mu, \epsilon, \sigma)$.

\footnotetext{
${ }^{*} \mathrm{E}_{\theta}, \mathrm{H}_{\mathrm{r}}, \mathrm{H}, \mu, \epsilon$ become $-\mathrm{H}_{\theta^{\prime}}, \mathrm{E}_{\mathrm{r}^{\prime}}, \mathrm{E}_{\gamma^{\prime}} \epsilon, \mu$, respectively.
}

\section{Harmonic Analysis}

Another method to simplify Eqs (9) thru (11) is to assume that the magnetic-dipole source emits energy at a given frequency. This is consistent with anticipated applications. Noise problems are expected to make time-dependent analysis (for example, from a step-function current pulse) very difficult in a gaswell logging operation. On the other hand, a source that emits several pulse trains, each in a narrowfrequency band and coupled with appropriate receiver filters, has greater potential for accurate analysis. The range of frequencies emitted by the source might take advantage of some frequency-dependent characteristic of the soil. Here we assume the time dependence of the source is $\exp (-i \omega t)$. Hence $\partial / \partial t$ becomes $-i \omega$, and for constant $\omega$, Eqs (9) thru (11) become three equations in three unknowns $B_{r^{\prime}} B_{z^{\prime}} E_{\theta}$. Note that a known $E_{\theta}(r, z)$ allows immediate calculation of $B_{r}, B_{z}$ by means of differentiation. Knowledge of $B_{r}$ or $B_{z}$ requires solution of another differential equation before the other two dependent variables can be determined. Elimination of $B_{r}$ and $B_{z}$ yields

$$
\frac{\partial^{2} E}{\partial z^{2}}+\frac{\partial^{2} E}{\partial r^{2}}+\frac{1}{r} \frac{\partial E}{\partial r}+\left(k^{2}-\frac{1}{r^{2}}\right) E=0
$$

where the $\theta$ subscript on $E$ is now omitted and where

$$
\mathrm{k}^{2} \equiv \mu \epsilon \omega^{2}+\mathrm{i} \omega \mu \sigma
$$

We now have one second-order elliptic differential equation to solve.

\section{Harmonic Magnetic Dipole in a Homogeneous Isotropic Medium}

The only characteristics of the source formally introduced thus far are its harmonic time dependence and its azimuthal symmetry. We now assume a magnetic-dipole source. The resulting electromagnetic fields in a homogeneous isotropic medium have been developed in several standard electromagnetictheory texts. If the medium is characterized by complex propagation constant $k$,

$$
\mathrm{E}=\frac{\mathrm{Mk} \mathrm{k}^{2}}{4 \pi} \sqrt{\frac{\mu}{\epsilon}} \frac{\mathrm{ir}}{\mathrm{k} \rho^{3}} \mathrm{e}^{\mathrm{ik} \rho}(1-\mathrm{ik} \rho),
$$

where

$$
\rho \equiv\left[\left(z-z_{c}\right)^{2}+r^{2}\right]^{1 / 2}
$$


and where $\mathrm{M}$ is the magnetic-dipole moment. Usual multipole expansions that lead to the magneticdipole term occur in spherical coordinates. We have converted to our earlier cylindrical coordinates. The dipole is located at $\left(z=z_{c}, r=0\right)$.

The magnetic-dipole moment of a coil of turns ( $T$ ), in a circular loop of radius (a), carrying current (I), is given by

$$
\mathrm{M}=\mathrm{TI} \pi \mathrm{a}^{2} .
$$

Time dependence enters the problem by means of the current

$$
I=I_{o} e^{-i \omega t} .
$$

If the medium is reasonably uniform near the source, then we expect that near the source the electric-field intensity is dominated by source contributions. Hence, as an approximation near the source, Eq (14) is appropriate. Later in the development this approximation is used as a boundary condition in the region immediately surrounding the source dipole.

\section{Formulation of Finite-Difference Equations in a Heterogeneous Medium}

Near the source region the $1 / \rho^{3}$ term will introduce quite large electric-field intensities. Solutions to the differential Eq (12) should be better behaved if the order of the singularity at $z=z_{c}, r=0$ can be reduced. To remove a portion of this rapid variation near the source, let

$$
\mathrm{S}=\mathrm{E} \rho^{2} / \mathrm{r}
$$

Equation (12) becomes

$$
\begin{gathered}
\frac{\partial^{2} S}{\partial z^{2}}+\frac{\partial^{2} S}{\partial r^{2}}+\frac{\partial S}{\partial r}\left[\frac{3}{r}-\frac{4 r}{\alpha}\right]+\frac{\partial S}{\partial z}\left[\frac{4\left(z_{c}-z\right)}{\alpha}\right] \\
+\left(k^{2}-\frac{2}{\alpha}\right) S=0
\end{gathered}
$$

where

$$
\alpha \equiv\left(z-z_{c}\right)^{2}+r^{2}=\rho^{2} .
$$

Although the differential equation is more involved, the solution should vary less rapidly with $\mathrm{r}$ and $\mathrm{z}$ and is expected to be easier to solve. In a finite-difference approach, the smoother variation of $S$ should allow larger step sizes $\Delta r, \Delta z$ than are possible with direct solution for $\mathrm{E}$.

A series of other variable changes are possible. For example, the choice

$$
\mathrm{W}=\mathrm{E} \rho^{3} / \mathrm{r}
$$

leads to the elliptic differential equation

$$
\begin{gathered}
\frac{\partial^{2} W}{\partial z^{2}}+\frac{\partial^{2} W}{\partial r^{2}}+\frac{\partial W}{\partial r}\left[\frac{3}{r}-\frac{6 r}{\alpha}\right]+\frac{\partial W}{\partial z}\left[\frac{6\left(z_{c}-z\right)}{\alpha}\right] \\
+k^{2} W=0 .
\end{gathered}
$$

Here we proceed with the solution in terms of $S$.*

Let the earth surrounding the source be divided into a series of horizontal slabs given by the interfaces $z_{i}=(i-1) \Delta z$, where $1 \leq i \leq i_{\max }$. Let the earth be further divided by a series of cylindrical surfaces with their vertical axes through the source. The source center is located at $z_{c}=\left(i_{c}-1\right) \Delta z$. The cylindrical surfaces are at $r_{j}=(j-1) \Delta r$, where $1 \leq j$ $\leq \mathrm{j}_{\max }$.

A vertical slice of the geometry is schematically illustrated in Figure 2. There is azimuthal symmetry about the $z$ axis.

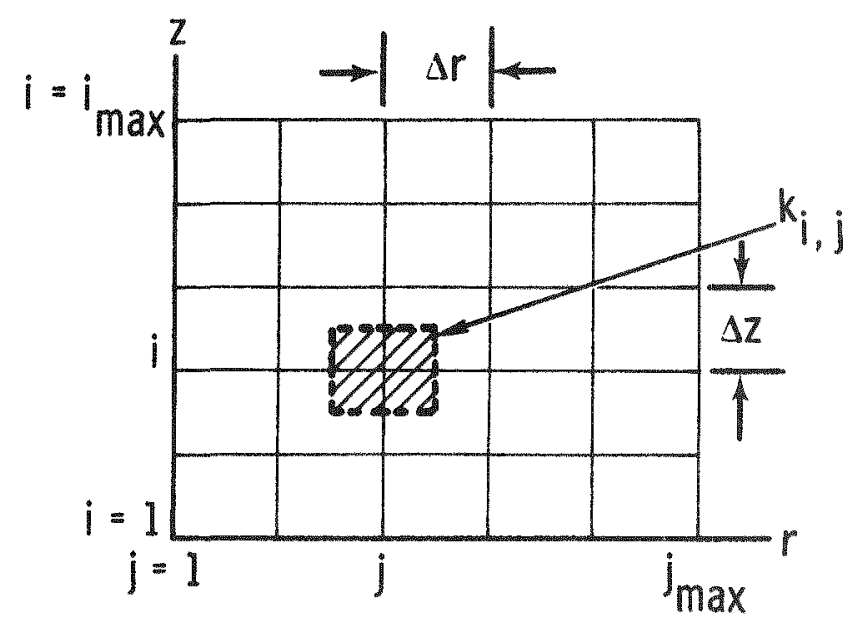

Figure 2. The Finite-Difference Grid

\footnotetext{
* This choice makes $S$ approximately constant at the outer bound. ary if the boundary can be placed at large $p$. The outer boundary conditions are then easier to treat.
} 
The intersection of grid lines $i$ and $j$ identifies the point of definition for $S_{1, j}$; i.e., the value of $S$ at position $z=(i-1) \Delta z$ and $r=(j-1) \Delta r$. At position i,j the approximations

$$
\begin{aligned}
& \frac{\partial S}{\partial \mathrm{r}}=\frac{S_{1, j+1}-S_{1,1-1}}{2 \Delta \mathrm{r}}, \\
& \frac{\partial S}{\partial z}=\frac{S_{1+1, j}-S_{1-1, j}}{2 \Delta z} \\
& \frac{\partial^{2} S}{\partial \iota^{2}}=\frac{S_{1+1,1}-2 S_{1, j}+S_{1-1,1}}{(\Delta z)^{2}} \\
& \frac{\partial^{2} S}{\partial r^{2}}=\frac{S_{1,+1}-2 S_{1, j}+S_{1, j-1}}{(\Delta r)^{2}},
\end{aligned}
$$

convert Eq (19) into

$$
\begin{aligned}
\beta_{0} \mathrm{~S}_{1,1}= & \beta_{1} \mathrm{~S}_{1+1, j}+\beta_{2} \mathrm{~S}_{1-1,1} \\
& +\beta_{3} \mathrm{~S}_{1, j+1}+\beta_{4} \mathrm{~S}_{1,-1}
\end{aligned}
$$

with

$$
\begin{aligned}
& \beta_{0}=\frac{2}{\Delta \mathrm{r}^{2}}+\frac{2}{\Delta \mathrm{z}^{2}}+\frac{2}{\alpha}-\mathrm{k}^{2}, \\
& \beta_{1}=\frac{1}{\Delta \mathrm{z}^{2}}-\frac{2\left(\mathrm{z}-\mathrm{z}_{\mathrm{c}}\right)}{\alpha \Delta \mathrm{z}}, \\
& \beta_{2}=\frac{1}{\Delta \mathrm{z}^{2}}+\frac{2\left(\mathrm{z}-\mathrm{z}_{\mathrm{c}}\right)}{\alpha \Delta \mathrm{z}}, \\
& \beta_{3}=\frac{1}{\Delta \mathrm{r}^{2}}+\frac{1}{2 \Delta r}\left[\frac{3}{\mathrm{r}}-\frac{4 \mathrm{r}}{\alpha}\right], \\
& \beta_{4}=\frac{1}{\Delta \mathrm{r}^{2}}-\frac{1}{2 \Delta r}\left[\frac{3}{\mathrm{r}}-\frac{4 \mathrm{r}}{\alpha}\right], \\
& \mathrm{r}=(\mathrm{j}-1) \Delta \mathrm{r}, \\
& \mathrm{z}=(\mathrm{i}-1) \Delta \mathrm{z}, \\
& \alpha=\left[\left(\mathrm{i}-\mathrm{i}_{\mathrm{c}}\right)^{2} \Delta \mathrm{z}^{2}+(j-1)^{2} \Delta \mathrm{r}^{2}\right] .
\end{aligned}
$$

The value of $k^{2}$ in $\beta_{\mathrm{o}}$ is the local value of $k^{2}$ at position $\mathrm{i}, \mathrm{j}$.

In Figure 2 each intersection is characterized by a uniform propagation constant $k_{1, j}$ that is modeled as constant within the rectangle of dimensions $\Delta r, \Delta z$ centered on the intersection point. Suppose there are $\mathrm{N}$ interior mesh intersections i,j. Equation (21) then represents $\mathrm{N}$ linear equations in the $\mathrm{N}$ unknowns $\mathrm{S}_{1, j}$. Values at the exterior mesh intersections (boundaries) must be specified separately.

\section{Successive Overrelaxation (SOR) Solution--Limitations}

Equation (21) suggests an iteration scheme for finding $S(z, r)$. At the $k$ th iteration

$$
\begin{aligned}
\bar{S}_{1, j}^{k}= & \frac{1}{\beta_{0}}\left[\beta_{1} S_{1+1, j}^{k-1}+\beta_{2} S_{1-1, j}^{k}\right. \\
& \left.+\beta_{3} S_{1, j+1}^{k-1}+\beta_{4} S_{1, j-1}^{k}\right] .
\end{aligned}
$$

This scheme indicates that the latest estimates of $S$ are employed as soon as they are available. This approach is called the Gauss-Seidel method. The SOR solution is given by

$$
S_{1,1}^{k}=S_{1,1}^{k-1}+p\left(\bar{S}_{1,1}^{k}-S_{1,1}^{k-1}\right) .
$$

The relaxation parameter is designed by p; value 1 returns to the Gauss-Seidel method. Values greater than 1 indicate overrelaxation; less than 1, underrelaxation. After an initial guess at all interior values $S_{1,,^{\prime}}$ iteration continues until convergence.

Several numerical experiments were conducted to test this method. A uniform isotropic medium was selected with (Dirichlet) boundary values (specifying S) obtained from Eqs (14) and (18). An initial guess of the exact solution was recognized as the solution. The small changes that did occur were quite stable by the $100^{\text {th }}$ iteration. An initial guess of $(0,0)$ for the real and imaginary parts of $\mathrm{S}$ at interior points led to slow convergence to the correct value at interior points. About 500 iterations were necessary for the solution to reach convergence. Limitations were also found on $\Delta r, \Delta z$, and $p$; for example, the following conditions led to a divergent solution: $\Delta r=\Delta z=0.06 \mathrm{~m}$, or $\mathrm{p} \geq$ 1.75. Convergence occurred for all tests with $1 \leq p \leq$ 1.5 when $\Delta \mathrm{r}=\Delta \mathrm{z}=0.01 \mathrm{~m}$. All combinations of these parameters were not tried. However, $\Delta r$ and $\Delta z$ must be sufficiently small that neighboring values of $S$ near the source vary by only a small amount $(\leqslant 5 \%)$.

Another limitation expected is that the spatial increments must be small enough to follow standing waves that might be excited between various layers 
in a problem. However, even at $10^{7} \mathrm{~Hz}$, wavelengths are large compared to $0.01 \mathrm{~m}$, and no additional restrictions are expected.

\section{Relaxation Convention}

The relaxation convention used in the early tests proceeded through the matrix by selecting $i=1$, updating all $j$ values starting at the boundary, then incrementing $i$ by 1 and proceeding through the $j$ values, with repetition until all $i, j$ values are processed. Sheldon ${ }^{2}$ suggests that the relaxation process can be improved by passing up the matrix of $i, j$ values, choosing only values for $i+j$ as an odd number; then passing downward from the top of the matrix and updating the values of $S$ for $i+j$ as an even number. As indicated by Boers, ${ }^{3}$ this permits corrections to propagate more rapidly in all directions and helps reduce systematic errors. Other alternatives are of course possible, such as starting with $i, j$ values around the source and propagating outward from there. If Sheldon's convention is used and then compared with the alternative approach in the solution for Test Problem A (discussed later), the alternative method appears favored. However, there are acceleration schemes intended to speed convergence of SOR methods by altering the relaxation parameter p. Numerical experiments indicate that these schemes do not lead to convergence in the earlier method. As discussed in Appendix A, they may speed convergence when Sheldon's relaxation convention is used.

\section{Boundary Conditions}

In a problem with several layers of earth, each with differing electrical parameters, there is no convenient equation analogous to Eq (14) that will give the appropriate values of $S$ on the boundary. If the solution space could be extended to infinity, the boundary $S$ is zero and no difficulty occurs. Unfortunately, limited computer storage prevents such a simple approach. In this finite-difference approach we must furnish boundary conditions to limit the number of unknowns to N.

Elliptic differential equations have a unique solution inside a closed region if the dependent variable (S) or its normal derivative $(\partial S / \partial n)$ is specified at each point on the boundary. Specifying $S(\partial S / \partial n)$ gives us Dirichlet (Neumann) boundary conditions. If different portions of the boundary are specified by different types of boundary conditions, the conditions are said to be mixed.
Inside a region immediately surrounding the source (the source region), the values of $S$ are obtained from Eqs (14) and (18). Along the $\mathrm{r}=0$ axis the electric-field intensity is zero (see Eq. (11)). Since

$$
\frac{\partial}{\partial r}\left(\frac{\rho^{2}}{r}\right)=1 \text { and } \frac{\partial \rho}{\partial r}=\frac{r}{\rho}=0
$$

at $r=0$, we find from $E q(14)$ that the magnetic dipole in a uniform medium satisfies

$$
\frac{\partial S}{\partial r}=0 \quad \text { at } r=0 .
$$

However, is this an appropriate boundary condition when layering occurs?

Boundary layers effectively produce virtual sources that contribute to the electric-field intensity. To maintain azimuthal asymmetry, all these virtual sources must be symmetric. Any sources on the $r=0$ axis (as produced by horizontal layering) give $\partial S / \partial \mathrm{r}$ $=0$ on the axis. An element of a virtual source at $(\mathrm{r}, \mathrm{z}, \phi)$ might contribute to $\mathrm{E}$ on the axis. However, a balancing element of the source at $(r, z, \phi+\pi)$ maintains $\mathrm{E}=0$ and $\partial \mathrm{S} / \partial \mathrm{r}=0$ at $\mathrm{r}=0$.

The outer boundaries $z=z_{\min }, z=z_{\max }$ and $r=r_{\max }$ remain to be treated. We accept that exact boundary conditions are not available and that the value of $S$ at these boundaries will be much more sensitive to layering effects and choices of electrical parameters than will the form of $\partial S / \partial n$. We attempt to formulate $\partial S / \partial \mathrm{n}$ such that actual dependence upon layering and upon the choices for $\epsilon, \mu$, and $\sigma$ is minimized.

As introduced earlier, a harmonic magnetic dipole in a homogeneous isotropic medium has

$$
\mathrm{S}=\mathrm{A} \frac{\mathrm{e}^{\mathrm{kk} \rho}}{\rho}(1-\mathrm{ik} \rho)
$$

where

$$
\mathrm{A}=\frac{\mathrm{Mki}}{4 \pi} \sqrt{\frac{\mu}{\epsilon}} .
$$

At the boundaries we require $\partial S / \partial z$ and $\partial S / \partial r$. Near the outer boundaries the electrical parameters are assumed independent of $r, z$. Then at the radial boundary,

$$
\frac{\partial S}{\partial r}=S f_{1}(r, z)
$$


where

$$
f_{1}=\frac{i r}{\rho^{2}}\left[\frac{i k \rho+k^{2} \rho^{2}-1}{k \rho+i}\right]
$$

At this outer boundary a finite-difference approximahon to the normal derivative becomes

$$
\frac{S_{1 \max }-S_{11}}{\Delta r}=\frac{S_{1 \max }+S_{11}}{2} f_{1}
$$

or

$$
S_{j \max }=S_{j 1} \frac{\left(1+f_{1} \Delta r / 2\right)}{\left(1-f_{1} \Delta r / 2\right)},
$$

where $\mathrm{j} 1=\mathrm{jmax}-1$. At such boundary points $\mathrm{r}=$ $(\mathrm{jmax}-1.5) \Delta \mathrm{r}, \mathrm{z}=(\mathrm{i}-1) \Delta \mathrm{z}, \rho=\left[\mathrm{r}^{2}+\left(\mathrm{z}-\mathrm{z}_{\mathrm{c}}\right)^{2}\right]^{1 / 2}$, and the value of $k$ is that appropriate for the mesh point ( $i$, jmax). At large values of $\rho$, the $\mathrm{rk} / \rho$ dependence and the small value of $\Delta r$ indicate $f_{1} \Delta r / 2$ should be small compared to 1 .

With a layered earth, each virtual source will contribute to $S$ at this boundary, with the form of the contribution similar to $\mathrm{Eq}(25)$. Each $\mathrm{f}_{1}$ depends only upon geometrical factors ( $k, r$, and $\rho$ ). Then Eq (25) applies in the layered earth also (with $f_{1}$ replaced by an effective value $f_{1 \mathrm{e}}$ ).

Consider the $z$ boundaries. Equation (22) gives

$$
\frac{\partial S}{\partial z}=S \frac{i}{\rho} \frac{\partial \rho}{\partial z}\left[\frac{i k \rho+k^{2} \rho^{2}-1}{i+k \rho}\right],
$$

where $\partial \rho / \partial z=\left(z-z_{c}\right) / \rho$. In the earlier form

$$
\frac{\partial S}{\partial z}=S f_{2}(r, z)
$$

where

$$
f_{2}=\frac{i\left(z-z_{c}\right)}{\rho^{2}}\left[\frac{i k \rho+k^{2} \rho^{2}-1}{i+k \rho}\right] .
$$

At $z=z_{\max }$ with $\mathrm{i} 1=\mathrm{imax}-1$,

$$
S_{1 \max }=S_{11} \frac{\left(1+f_{2} \Delta z / 2\right)}{\left(1-f_{2} \Delta z / 2\right)}
$$

At $z=z_{m i n}$ the change in the sign of $z-z_{c}$ leads to

$$
S_{1 \mathrm{~min}}=S_{12} \frac{\left(1-f_{2} \Delta z / 2\right)}{\left(1+f_{2} \Delta z / 2\right)}
$$

where $\mathrm{i} 2$ is 2 . The minimum $\mathrm{i}$ (imin) is always 1 .
A problem with reflection symmetry about $z=z_{c}$ could take advantage of the symmetry and solve the problem in only the upper half of the spatial mesh. In that case, $\partial S / \partial z=0$ at $z=z_{c}$ would be a convenient boundary condition along the lower $(i=1)$ boundary. However, the naturally occurring horizontal layering is rarely symmetric about the source depth.

\section{Axial Boundary Condition}

The axial boundary condition is $\partial S / \partial r=0$ at $r=$ 0 . Several methods can be used to set $S(I, 1)$ to satisfy the requirement. A simple possibility is $S(\mathbb{I}, 1)=S(I, 2)$ that results from $\partial S / \partial r \approx\left(S_{1,1+1}-S_{1, j}\right) / \Delta r$. However, better choices exist. Earlier we found $\partial S / \partial r=\mathrm{Sf}_{1}(r, z)$ in $\mathrm{Eq}(23)$, with

$$
f_{1}=\frac{i r}{\rho^{2}}\left[\frac{i k \rho+k^{2} \rho^{2}-1}{k \rho+i}\right]
$$

When $r$ is within $\Delta r$ of 0 , with $z$ values outside the source region (i.e., $\left|z-z_{c}\right|=$ several times $\Delta z$ ) and with $\partial \mathrm{k} / \partial \mathrm{r}=0$ in the vicinity of $\mathrm{r}=0$ for each value of $I$, then the $r$ variation near $r=0$ is described by $f_{1}$ $\approx$ constant $x$ r. Hence

$$
\frac{\partial S}{\partial \mathrm{r}} \approx \mathrm{C}_{1} \mathrm{Sr}
$$

with $C_{1}$ a constant. With $S$ approximately constant $(\partial S / \partial r=0$ at $r=0)$

$$
\mathrm{S} \approx \mathrm{S}(\mathrm{r}=0)+\mathrm{C}_{2} \frac{\mathrm{r}^{2}}{2}
$$

with constant $C_{2}$. In finite-difference form

$$
\mathrm{S}_{1,2}=\mathrm{S}_{1,1}+\text { Constant } \frac{\Delta \mathrm{r}^{2}}{2}
$$

Applied between I values 2 and 3

$$
S_{1,3}-S_{1,2}=\text { Constant }\left[\frac{(2 \Delta r)^{2}-(\Delta r)^{2}}{2}\right]
$$

The combination yields

$$
S_{1,1}=\frac{4 S_{1,2}-S_{1,3}}{3}
$$

In one numerical exercise the iterations became unstable. The source of the difficulty was along the $r$ $=0$ axis. Evidently the initial guess for $S_{1,1}$ was too far from the correct solution for convergence to occur. In this case a change of the $r=0$ boundary condition to 


$$
S_{1,1}=\frac{9 S_{1,2}-S_{1,3}}{8}
$$

produced convergence. This second choice weights the $j=2$ value move heavily; i.e., closer to the simple choice of $S_{1,1}=S_{1,2}$ noted earlier. Either Eq (30) or (31) is used for the axial boundary condition for all results presented in this report.

With $\partial S / \partial r \approx C_{1} S r$, the boundary condition can also be presented in the form $1 \mathrm{n} S=\int \mathrm{f}_{1} \mathrm{dr}+\mathrm{C}_{3}$, or $S$ $=C_{4} \exp (\alpha r)$; i.e., a "skin depth" type of behavior. However, at $r=0 \partial S / \partial r \neq 0$ in that approximation and results are not as satisfactory.

\section{Alternative Form for Outer Boundary Conditions}

Earlier the outer boundary conditions at $r_{\max }$ were given by $\partial S / \partial \mathrm{r}=\mathrm{Sf}_{1}$ and

$$
S_{1, \text { max }}=S_{1, \text { max }-1} \frac{\left(1+f_{1} \Delta r / 2\right)}{\left(1-f_{1} \Delta r / 2\right)}
$$

The $f_{1}$ is evaluated by geometric factors that are exact when all real and virtual sources are located at the source dipole. It may be possible to obtain a local estimate for $f_{1}$ (i.e., $f_{1 e}$ ) that only uses neighboring values of $S_{1, j}$. For example,

$$
\begin{aligned}
& \frac{S_{1, \text { max }}-S_{1, \text { max }-2}}{2 \Delta r} \approx S_{1, j \max -1} f_{1 e} \\
& \frac{S_{1, \text { max }}-S_{1, \text { max }-1}}{\Delta r} \approx \frac{S_{1, \text { max }}+S_{1, j \max -1}}{2} f_{1 e} .
\end{aligned}
$$

Elimination of $f_{1 \mathrm{e}}$ yields

$$
\begin{aligned}
2 S_{1, \text { max }}= & 3 S_{1, \max -1}+S_{1,1 \max -2}-\left[S_{1, \max -2}{ }^{2}\right. \\
& +10 S_{1, \text { max }-1} S_{1, \text { max }-2} \\
& \left.-7 S_{1, \max -1}\right]^{1 / 2} .
\end{aligned}
$$

In like manner the $z$ boundaries give

$$
\begin{aligned}
2 S_{1 \max , 1}= & 3 S_{1 \max -1, j}+S_{1 \max -2, j}-\left[S_{1 \max -2, j}{ }^{2}\right. \\
& +10 S_{1 \max -1} S_{1 \max -2} \\
& \left.-7 S_{1 \max -1}^{2}\right]^{1 / 2}
\end{aligned}
$$

$$
\begin{aligned}
2 S_{1, j}= & 3 s_{2,3}+S_{3,1}-\left[S_{3,1}{ }^{2}\right. \\
& \left.+10 s_{2,3} s_{3,3}-7 S_{2,3}{ }^{2}\right]^{1 / 2} .
\end{aligned}
$$

Several test runs with these boundary conditions indicated no particular advantage. However, special cases may occur where they may be useful. If difficulty is experienced in convergence for a specific problem and the difficulty is traced to outer boundary conditions, these alternatives are offered.

\section{Test for Convergence}

In the general problem an exact solution is not available. Hence a test for convergence is required to terminate the iteration scheme. The value of $S$ at a specific point in the $z, r$ mesh at iteration number $t$ is designated by $S_{1, j}^{t}$. Sheldon ${ }^{2}$ has suggested using $S_{s}$, the sum of squares of differences

$$
S_{s} \equiv \sum_{1, j}\left|S_{1, j}^{t}-S_{1, j}^{t-1}\right|^{2} \zeta_{1, j}
$$

as a test for convergence. $S_{\mathrm{s}}$ less than a designated number would indicate sufficient convergence. Here $\zeta_{1,1}=0$ at the boundary points and inside the source region. Elsewhere $\zeta_{1,1}=1$. The number of $i, j$ combinations that can contribute to the sum is

$$
N_{1, j}=\sum_{1, j} \zeta_{1, j}
$$

To eliminate dependence upon the number of $i, j$ combinations, we calculate the average of the summation of squares of differences

$$
S 1=N_{1,1}^{-1} S_{s}
$$

One numerical example indicated reasonable convergence when $\mathrm{S} 1<4$. E - 14. More general normalization might include division by the square of the following quantities: the dipole current, the number of turns on the dipole loop, and the dipole radius squared $/(0.01 \mathrm{~m})^{2}$. The frequency and the electrical parameters in the ground also influence a reasonable choice for the $\mathrm{S} 1$ limit. Hence, in applications, results from more than one iteration must be examined to determine if convergence is sufficient for your problem. 


\section{Nonuniform Radial Mesh}

In some applications it may be convenient to use a nonlinear radial mesh. Discontinuities in $\epsilon$ and $\sigma$ may be expected near the drillhole. Because the receivers are also located close to the $z$ axis, enhanced resolution is desired there. In addition, discontinuities far from the $z$ axis have less influence upon the received signal; hence it should be less vital to accurately model such discontinuity positions. We desire small $\Delta \mathrm{r}$ intervals close to $\mathrm{r}=0$, and larger $\Delta \mathrm{r}$ as $\mathrm{r}$ increases.

Let

$$
\frac{r}{r_{0}}=\left(\frac{t}{t_{0}}\right)^{\gamma}
$$

where $\gamma$ is a constant, greater than 1. We arbitrarily choose units of $\mathrm{r}$ and $\mathrm{t}$ to be measured in terms of the constants $r_{o}=1 \mathrm{~m}$ and $t_{o}=1 \mathrm{~s}$ to find

$$
\begin{aligned}
& \frac{\partial S}{\partial r}=\frac{\partial S}{\partial t} \frac{\partial t}{\partial r}=\frac{\partial S}{\partial t} \frac{t}{\gamma} . \\
& \frac{\partial^{2} S}{\partial r^{2}}=\frac{\partial^{2} S}{\partial t^{2}} \frac{t^{2-2 \gamma}}{\gamma^{2}}+\frac{\partial S}{\partial t}\left(\gamma^{-2}-\gamma^{-1}\right) t^{1-2 \gamma}
\end{aligned}
$$

With these substitutions, Eq (19) becomes

$$
\begin{gathered}
\frac{\partial^{2} S}{\partial \mathrm{z}^{2}}+\frac{\partial^{2} S}{\partial \mathrm{t}^{2}} \frac{\mathrm{t}^{2-2 \gamma}}{\gamma^{2}}+\frac{\partial S}{\partial \mathrm{z}}\left[\frac{4\left(\mathrm{z}_{\mathrm{c}}-\mathrm{z}\right)}{\alpha}\right] \\
+\frac{\partial S}{\partial \mathrm{t}} \frac{\mathrm{t}}{\gamma}\left[\left(\frac{1}{\gamma}+2\right) \mathrm{t}^{-2 \gamma}-\frac{4}{\alpha}\right] \\
+\left(\mathrm{k}^{2}-\frac{2}{\alpha}\right) \mathrm{S}=0
\end{gathered}
$$

The coefficients in $\mathrm{Eq}(21)$ are then altered to

$$
\begin{aligned}
& \beta_{0}=\frac{2}{\alpha}-\mathrm{k}^{2}+\frac{2}{\Delta \mathrm{z}^{2}}+\frac{2}{(\Delta \mathrm{t})^{2}} \frac{\mathrm{t}^{2-2 \gamma}}{\gamma^{2}} \\
& \beta_{1}=\frac{1}{\Delta \mathrm{z}^{2}}-\frac{2\left(\mathrm{z}-\mathrm{z}_{\mathrm{c}}\right)}{\alpha \Delta \mathrm{z}} \\
& \beta_{2}=\frac{1}{\Delta \mathrm{z}^{2}}+\frac{2\left(\mathrm{z}-\mathrm{z}_{\mathrm{c}}\right)}{\alpha \Delta \mathrm{z}} \\
& \beta_{3}=\frac{\mathrm{t}^{2-2 \gamma}}{\gamma^{2} \Delta \mathrm{t}^{2}}+\frac{\mathrm{t}}{\gamma}\left[\left(\frac{1}{\gamma}+2\right) \mathrm{t}^{-2 \gamma}-\frac{4}{\alpha}\right] \frac{1}{2 \Delta \mathrm{t}} \\
& \beta_{4}=\frac{\mathrm{t}^{2-2 \gamma}}{\gamma^{2} \Delta \mathrm{t}^{2}}-\frac{\mathrm{t}}{\gamma}\left[\left(\frac{1}{\gamma}+2\right) \mathrm{t}^{-2 \gamma}-\frac{4}{\alpha}\right] \frac{1}{2 \Delta \mathrm{t}}
\end{aligned}
$$

and the solution proceeds as before.
The $t$ mesh is

$$
t_{j}=(j-1) \Delta t
$$

In the computer code several variables are tabulated for the set of $j$ values.

$$
\begin{aligned}
& \operatorname{TM}(J)=t_{j}=(J-1) \Delta t \\
& \operatorname{RM}(J)=\operatorname{TM}(J) * * \text { GAMMA } \\
& \operatorname{TM} 2(J)=t_{j}^{2-2 \gamma} / \gamma^{2} \\
& \operatorname{TM} 1(J)=t_{j}^{1-2 \gamma}\left(\frac{1}{\gamma^{2}}-\frac{1}{\gamma}\right) \\
& \operatorname{TM} 3(J)=\frac{3}{\gamma} t_{j}^{1-2 \gamma} \\
& \operatorname{TM} 4(J)=\frac{4 t}{\gamma} .
\end{aligned}
$$

As an example of how the $\mathrm{T}$ mesh might be chosen, let $\gamma=1.1$. At $J=6$, set $r=0.02559 \mathrm{~m}$. Such a choice might be desired if a discontinuity in electrical conductivity occurs near this $\mathrm{r}$ value.

$$
\mathrm{r}=[(\mathrm{J}-1) \Delta \mathrm{t}]^{1.1}
$$

This furnished $\Delta t=0.00714142$. Then $\mathrm{J}=20$ gives the value $r=0.11112 \mathrm{~m}$ where a second discontinuity might be modeled. As noted in Figure 2, the grid assumes constant $k_{i, j}$ near each intersection $i, j$. Hence the $r$ value at a discontinuity in electrical parameters should correspond to the I value such that

$$
r=t^{\gamma}=[(J-1+0.5) \Delta t]^{\gamma}
$$

This places the discontinuity at a midpoint between two t values in the mesh.

\section{Test Problem A: Effect of Neumann Boundary Conditions Upon Numerical Solutions}

Numerical experiments test the SOR solution by using Neumann boundary conditions and the early relaxation scheme. Again, the magnetic dipole in a uniform medium is used for easy comparison with exact analytical results. Specification of $\partial S / \partial \mathrm{n}$ rather than $S$ at the outer boundaries increased the number of iterations required for convergence. The earlier test that used Dirichlet boundary conditions and an 
initial guess of $(0,0)$ required $\sim 500$ iterations for convergence. Here 800 iterations are insufficient for convergence. For example, with the problem parameters $\Delta \mathrm{r}=\Delta \mathrm{z}=0.01 \mathrm{~m}$; imax $=99 ; \mathrm{jmax}=99 ; \mu=$ $\mu\left(\mu_{\mathrm{o}}, 0\right) ; \epsilon=\left(\epsilon_{\mathrm{o}}, 0\right) ; \sigma=(1 \mathrm{mho} / \mathrm{m}, 0) ; \mathrm{p}=1.5$; dipole radius, $0.1 \mathrm{~m}$; dipole current amplitude, $1 \mathrm{~A}$; frequen$\mathrm{cy}, 20 \mathrm{kHz}$; the initial guess for $\mathrm{S}(0,0)$; and with the source region designated as within $0.1 \mathrm{~m}$ of the source, the calculated value of $S$ at $z=z_{c}$ (corresponding to $i=50$ ) and $r=0.17 \mathrm{~m}$ is indicated in Table 1.

Table 1. Illustration of Convergence for Magnetic Dipole in Uniform Medium

$\begin{array}{ccc}\text { Iteration } & \text { Real S } & \text { Imaginary S } \\ 0 & 0 & 0 \\ 50 & -0.21 \mathrm{E}-5 & +0.18 \mathrm{E}-2 \\ 100 & -0.28 \mathrm{E}-5 & +0.20 \mathrm{E}-2 \\ 200 & -0.33 \mathrm{E}-5 & +0.22 \mathrm{E}-2 \\ 800 & -0.42 \mathrm{E}-5 & +0.22 \mathrm{E}-2 \\ \text { Correct Value } & -0.51 \mathrm{E}-5 & +0.23 \mathrm{E}-2\end{array}$

Convergence is slower near the outer boundary. At $\mathrm{z}=\mathrm{z}_{\mathrm{c}}$ and $\mathrm{r}=0.98 \mathrm{~m}$, results are indicated in Table 2. Although the method is slow, it leads to the correct result.

Table 2. Convergence Near Boundary for Chosen Problem

$\begin{array}{ccc}\text { Iteration } & \text { Real S } & \text { Imaginary S } \\ 0 & 0 & 0 \\ 50 & -0.24 \mathrm{E}-5 & +0.31 \mathrm{E}-4 \\ 100 & -0.18 \mathrm{E}-5 & +0.20 \mathrm{E}-4 \\ 200 & -0.14 \mathrm{E}-5 & +0.19 \mathrm{E}-4 \\ 800^{*} & -0.72 \mathrm{E}-5 & +0.20 \mathrm{E}-3 \\ \text { Correct Value } & -0.25 \mathrm{E}-4 & +0.40 \mathrm{E}-3\end{array}$

${ }^{*}$ Convergence not attained

\section{Test Problem B: Earth With One Vertical Interface}

As the first example of an inhomogeneous earth, we chose an example treated by Moran and Kunz (MK). The fluid invasion zone is characterized by $\sigma_{\mathrm{i}}$ $=0.0625 \mathrm{mho} / \mathrm{m}$ and diameter $D_{i}=80 \mathrm{in} .(2.032 \mathrm{~m})$. Outside the invasion zone $\sigma=1 \mathrm{mho} / \mathrm{m}$. The conductivities are real, permittivity $=\epsilon_{\mathrm{o}}$, magnetic per meability $=\mu_{0}$. With $\Delta \mathrm{r}$ equal to $0.01 \mathrm{~m}$, jmax $\geq 102$ is necessary to include all the invasion zone in the problem. The large conductivity outside this invasion zone reduces the signal at larger distances. We attempt a solution with imax $=201 ;$ jmax $=201 ; \Delta z=$ $0.01 \mathrm{~m}$; source region $\mathrm{r}$ values within $0 \leq \mathrm{r} \leq 0.1 \mathrm{~m}$ and $z$ values within $0.05 \mathrm{~m}$ of the source; frequency, $20 \mathrm{kHz}$; and the S1 limit set at 3.E-17 in the initial convergence test. The receiver-coil radius (a) is taken as the radius of the source coil. The geometry is illustrated in Figure 3. Problem parameters are listed below and discussed in greater detail in Appendix $\mathrm{E}$.

$$
\begin{aligned}
\text { DZSOUR } & =0.10 \mathrm{~m} \\
\text { DRSOUR } & =0.10 \mathrm{~m} \\
\text { DT } & =0.01 \mathrm{~m} \\
\text { DZ } & =0.01 \mathrm{~m} \\
\text { NZ } & =201 \\
\text { NR } & =201 \\
R P & =1.9 \\
\text { TURNS } & =1 \\
\text { CURR } & =1 \\
\text { RADIUS } & =0.1 \mathrm{~m} \\
\text { GAMMA } & =1.0
\end{aligned}
$$

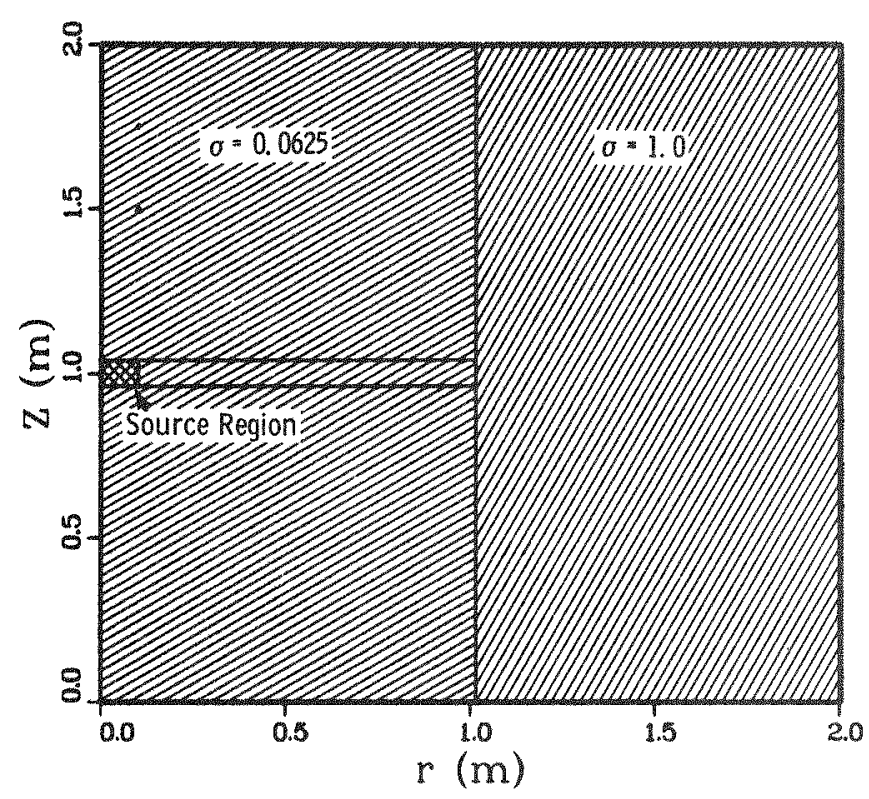

Figure 3. Geometry for MK Problem

Moran and Kunz define an apparent conductivity that serves as a useful parameter for comparison. The electric field will produce a voltage

$$
V=2 \pi a R E(L, a)
$$

on a receiver coil of $(R)$ turns wound on a mandrel of radius (a), at axial distance ( $L$ ) from the dipole source. The apparent conductivity, $\sigma_{\mathrm{a}}$ is defined by 


$$
\sigma_{\mathrm{a}}=-\mathrm{V}_{\mathrm{R}} / \mathrm{K}
$$

where the $R$ subscript indicates real part and where

$$
\begin{aligned}
& K \equiv \frac{(\omega \mu)^{2}\left(\pi a^{2}\right)^{2} \text { TRI }}{4 \pi L}, \\
& I \equiv \text { current magnitude } \\
& T \equiv \text { number of turns in the dipole } \\
& \mu \equiv \text { permeability of ground (assumed constant) } .
\end{aligned}
$$

\begin{tabular}{|c|c|c|c|c|}
\hline L & $\begin{array}{c}0.50 \mathrm{~m} \\
(19.7 \mathrm{in.})\end{array}$ & $\begin{array}{c}0.75 \mathrm{~m} \\
(29.5 \mathrm{in} .)\end{array}$ & S1 & $\begin{array}{c}\text { Number } \\
\text { of } \\
\text { Iterations }\end{array}$ \\
\hline$\sigma_{\mathrm{a}}(\mathrm{mho} / \mathrm{m})$ & 0219 & 0254 & $3 E-17$ & 300 \\
\hline $\begin{array}{l}a=010 \mathrm{~m} \\
\sigma_{1}=00625\end{array}$ & 0213 & 0247 & $35 E-18$ & 1600 \\
\hline $\begin{array}{l}\text { MK value of } \\
\sigma_{\mathrm{a}}(\mathrm{mho} / \mathrm{m})\end{array}$ & 0230 & 0310 & $\ldots$ & - \\
\hline
\end{tabular}

Results for $\sigma_{a}$ are compared in Table 3 after 300 and 1600 iterations.

Table 3. Apparent Conductivity in Test Problem B

Similar calculation for a homogeneous medium with $\sigma=1 \mathrm{mho} / \mathrm{m}$ results in values 0.8873 and 0.8519 $\mathrm{mho} / \mathrm{m}$ in good agreement with $\mathrm{MK}$ values. The results in Table 3 are not impressive. Let us examine the results more closely to determine if convergence has been attained.

The solid curve in Figure 4 indicates reasonable behavior of the convergence parameter and suggests the solution is relatively stable by 600 iterations. The estimated value of the apparent conductivity in Figure 5 indicates convergence is reasonable at the 1600 Iterations in Table 3 , where $\sigma_{a}$ values have $\sim 20 \%$ error. Even when convergence is attained; the error is large. After 1600 iterations, the maximum percent difference between successive values of the real part $S(I, J)$ is $0.00108 \%$. It occurs for $I=23$ and $J=4$.

Variation of $\sigma_{\mathrm{a}}$ with coil spacing is provided as the solid curve with $x$ data points in Figure 6; MoranKunz results are also indicated. The disagreement at larger $L$ values suggests that the outer boundary condition may be causing difficulty. To test this hypothesis, the calculation was repeated with the following changes:

$$
\begin{aligned}
\mathrm{DZ} & =0.02 \mathrm{~m} \\
\text { DZSOUR } & =0.20 \mathrm{~m}
\end{aligned}
$$

These results are shown as the dashed curves in Figures 4 through 6. After 1500 iterations the maximum percent difference between successive values of $S(I, J)$ is then $0.00060 \%$. It occurs for $I=16$ and $J=3$. These results are compared with the earlier calculation and with Moran-Kunz results in Figure 6 . At the positions $\mathrm{L}=0.5$ and $0.75 \mathrm{~m}$ in Table 3 , the converged values have become $\sigma_{\mathrm{a}}=0.233$ and 0.302 mho/ $\mathrm{m}$, in good agreement with the MK values in Table 3.

As an example of one type of difficulty that may be encountered, the three-dimensional (3-D) graph in Figure 7 indicates lack of symmetry upon reflection about the $z=z_{c}$ plane. It was encountered in an early calculation for Test Problem B. Physically, symmetry should be present. An error was discovered in a call to the EP subroutine developed for this problem that resulted in different $\sigma, t$ chosen inside the source region, depending upon the value of $z$. Hence the source itself was not symmetric. Plotting capabilities (by means of the DIPPL program discussed in Appendix F) provide quick visual checks for such obvious errors.

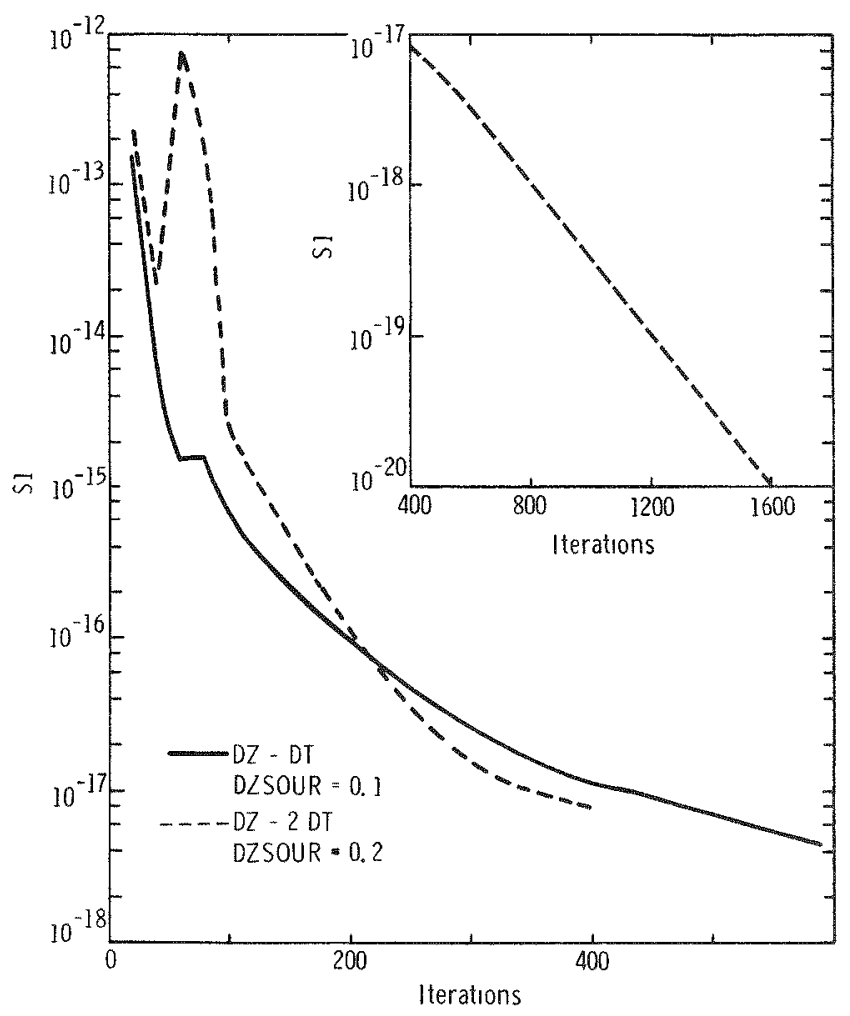

Figure 4. Variation of Convergence Parameter S1 With Iteration Number for Test Problem B 


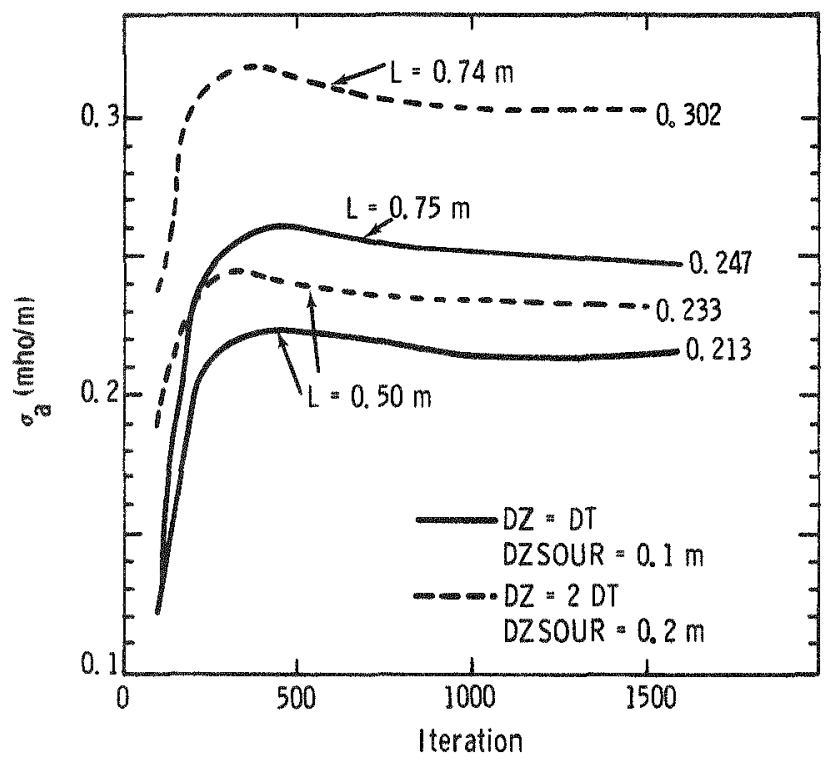

Figure 5. Apparent Conductivity at Two Positions in The Mesh of Test Problem B

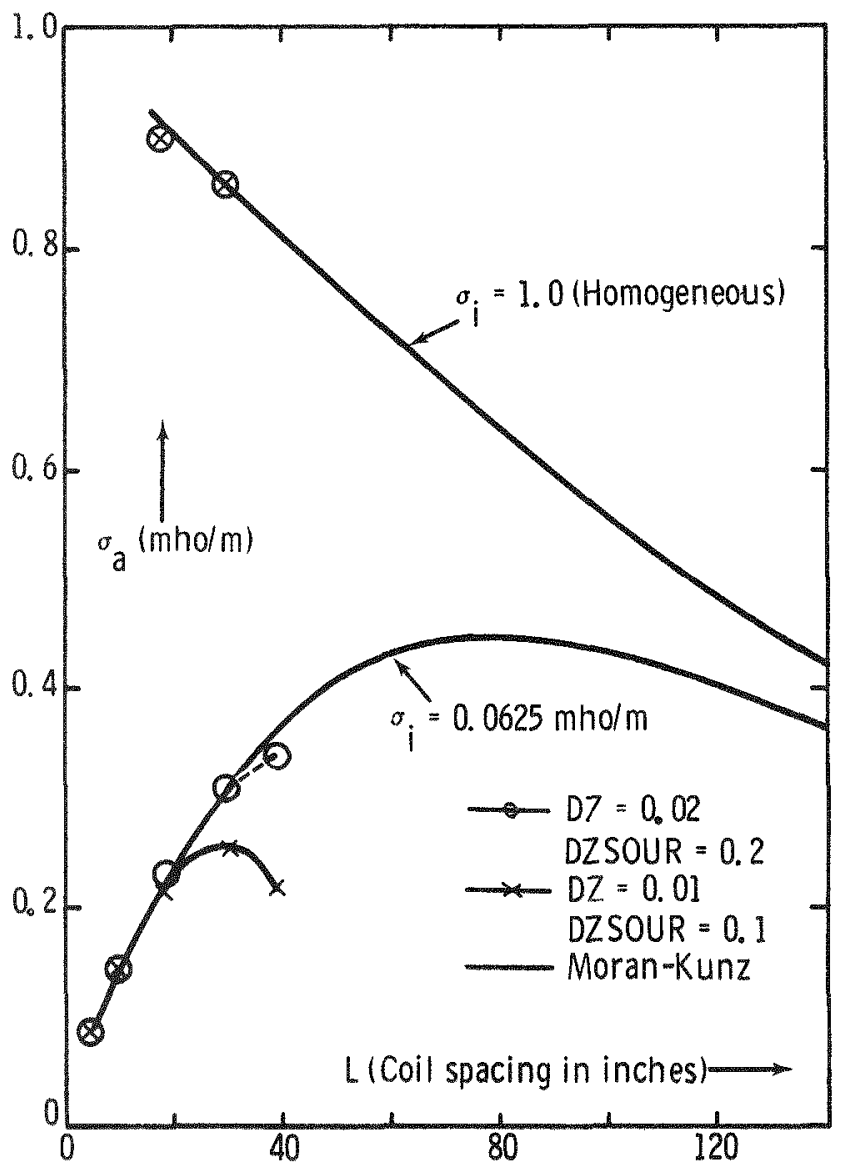

Figure 6. Apparent Conductivity vs Transmitter to Receiver-Coil Spacing (L) for Test Problem B

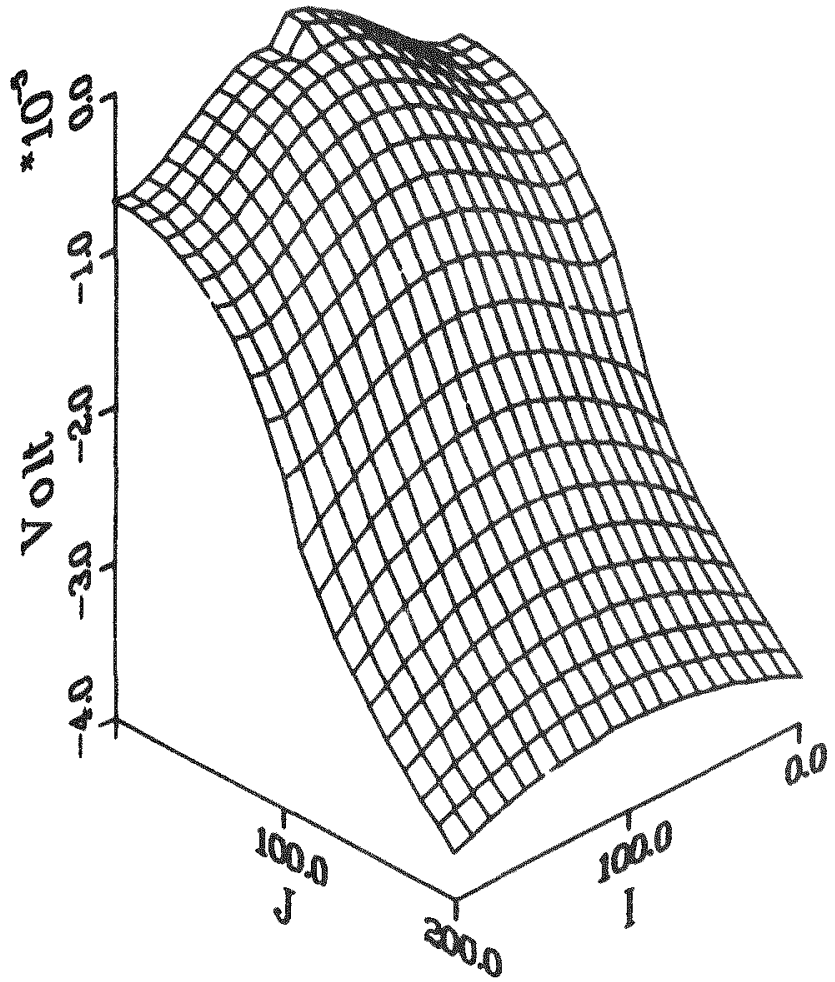

Figure 7. Real (S) Distribution as a Function of $x[=(I-1)$ DT $]$ and $f=(J-1) D Z]$ Illustrating an Error in the Source Region for an Early Calculation of Test Problem B

Plots generated by the DIPPL code for Test Problem $B$ are given in Figures 8 through 13 for the case of $\mathrm{DZ}=0.02 \mathrm{~m}$. At the $\mathrm{z}(\mathrm{I})$ boundaries of Figure 9 the alteration in slope is expected to cause the error encountered in Figure 6 at large $L$ values.

The three curves in Figures 10, 12, and 13 result from the voltage calculated for receiver coils of three radii in the mesh of $\mathrm{r}$ values where $\mathrm{r}=\mathrm{T}^{\gamma}$ and $\mathrm{T}(\mathrm{J})=$ $(J-1)$ DT. The solid curves correspond to the value of J given by

$$
\mathrm{J}_{\text {solid }}=\frac{(\text { RADIUS })^{1 / \gamma}}{D T}+1
$$

where RADIUS is the radius of the source dipole. The resulting $r$ is within one step (in $J$ ) of the source radius. The dashed and - - - curves correspond to I values $J_{\text {solid }}-1$ and $J_{\text {solid }}+1$, respectively. In Figure 13 the three curves are not distinguishable.

The open-circuit voltage in the coil is $2 \pi \mathrm{rE}(\mathrm{z}, \mathrm{r})$ for the receiver of radius $r$ at axial position $z$. Since $\partial S / \partial r$ $\sim 0$ near the axis, $S$ is approximately independent of $r$ for a small axial receiver a long distance from the dipole. Since $\mathrm{E}=\mathrm{Sr} / \rho^{2}$, the electrical field is then proportional to $r$, making the voltage proportional to 
$r^{2}$ The data in Figure 10 allow us to check this hypothesis At this low frequency, the phase alteration is neglected Table 4 indicates $V$ is proportional to $\mathrm{r}^{2}$, at least within the limits of accuracy for reading data in Figure 10

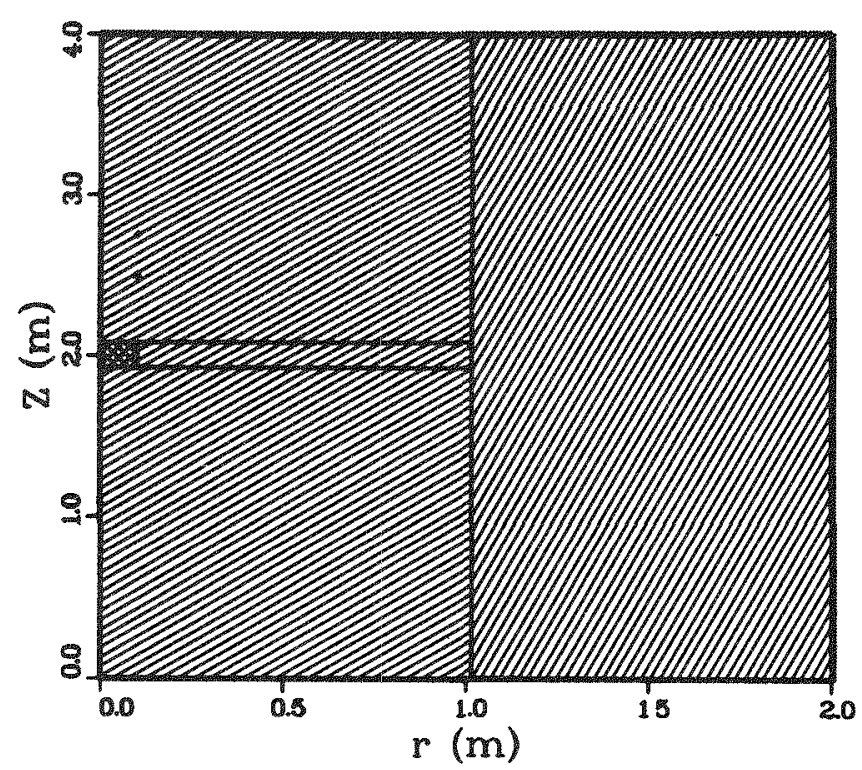

Figure 8 Soll Layering Geometry for Test Problem B

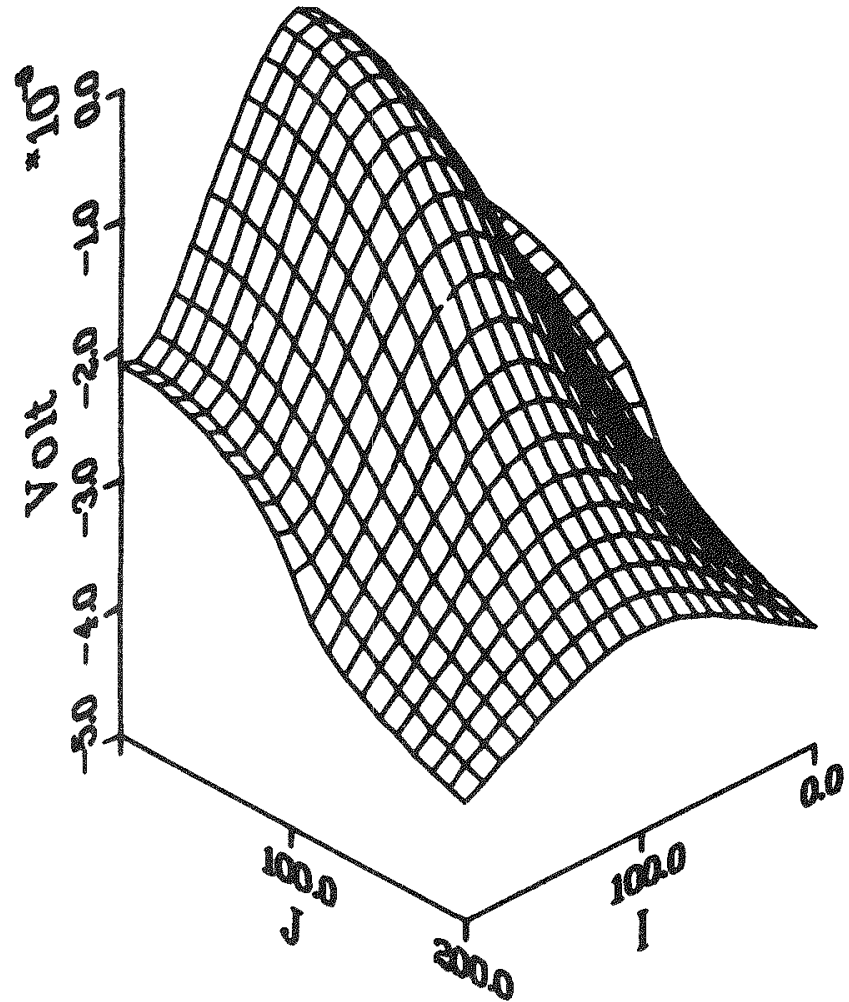

Figure 9 Real S( $/$ r) Distribution for Test Problem B
Table 4. Check of $\mathrm{r}^{2}$ Variation for Real Part of the Complex Coil Voltage at $L=0.76 \mathrm{~m}$

$\begin{array}{ccc}\mathrm{r}(\mathrm{m}) & \text { Real V }(\mathrm{V}) & \text { Real V/. } \mathbf{r}^{\mathbf{2}} \times \mathbf{1 0}^{7}\left(\mathrm{~V} / \mathrm{m}^{2}\right) \\ 009 & -735 \times 10^{-7} & 907 \\ 010 & -913 \times 10^{-7} & 913 \\ 011 & -1105 \times 10^{-7} & 913\end{array}$
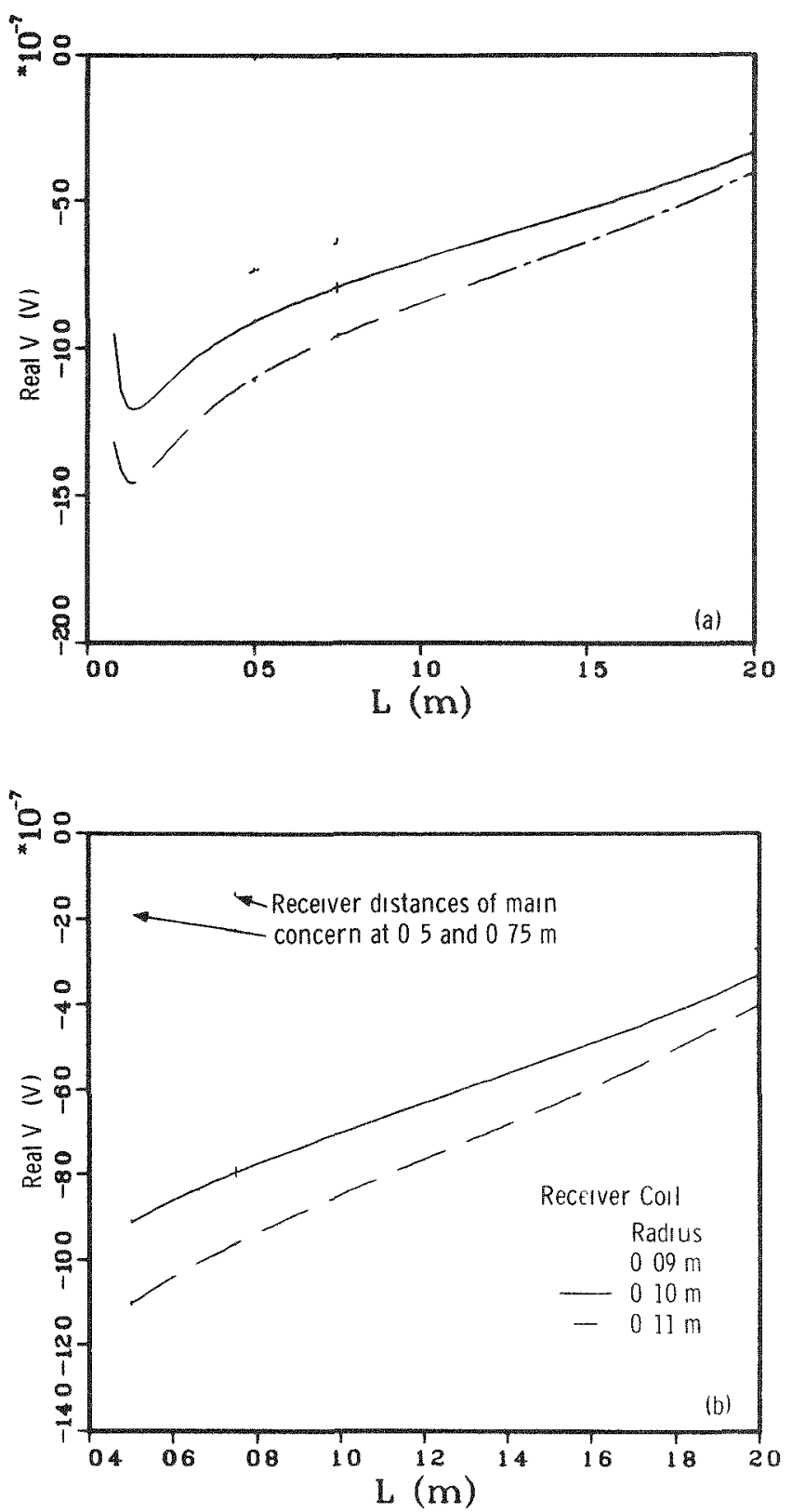

Figure 10 Real Part of Voltage in a Receiver Coll at Axial Distance $L$ From the Dipole Source in Test Problem B (Part b gives the variation in a more restricted range of $L$ values) 

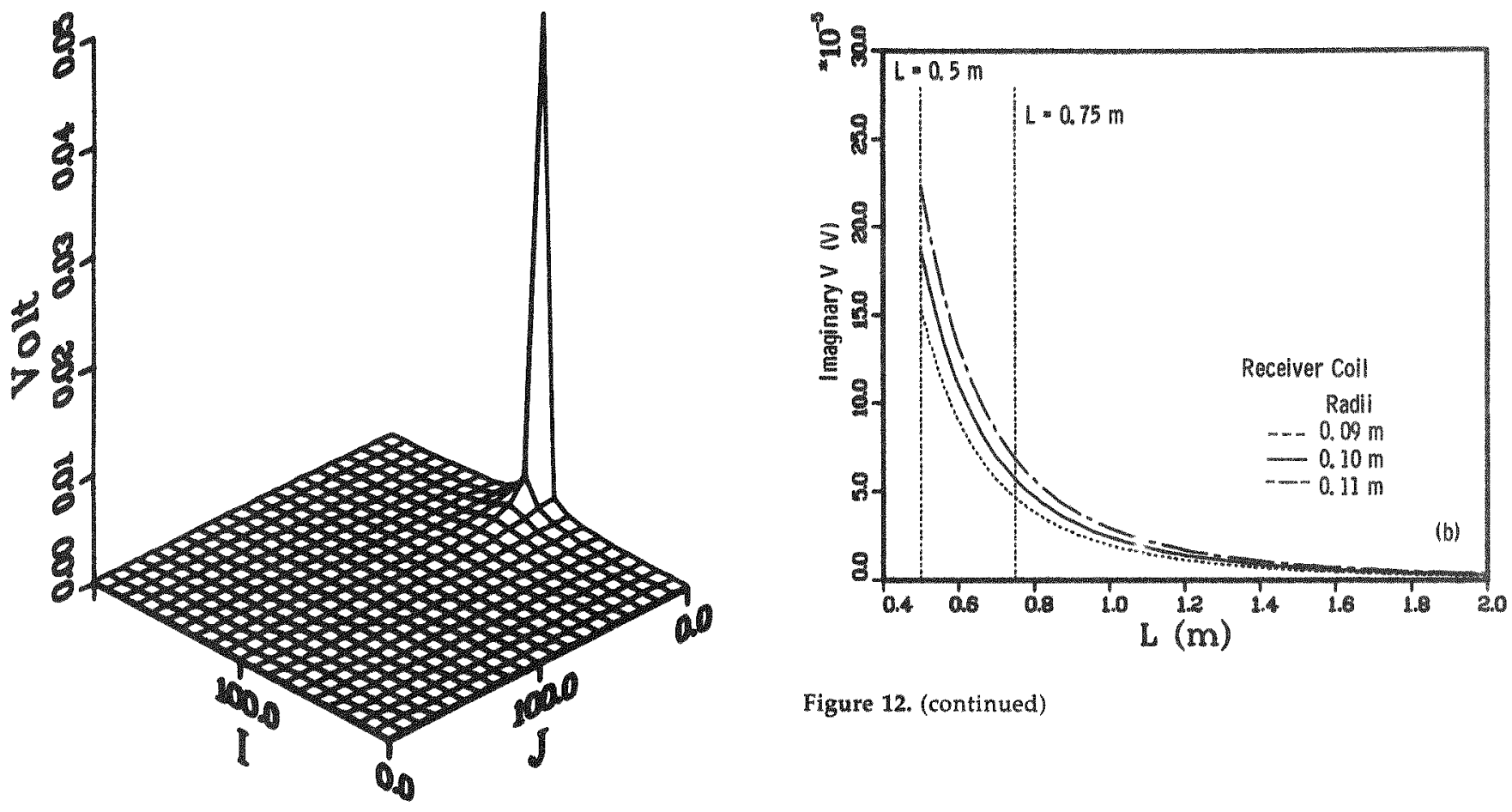

Figure 12. (continued)

Figure 11. Imaginary S(z,r) Distribution for Test Problem B

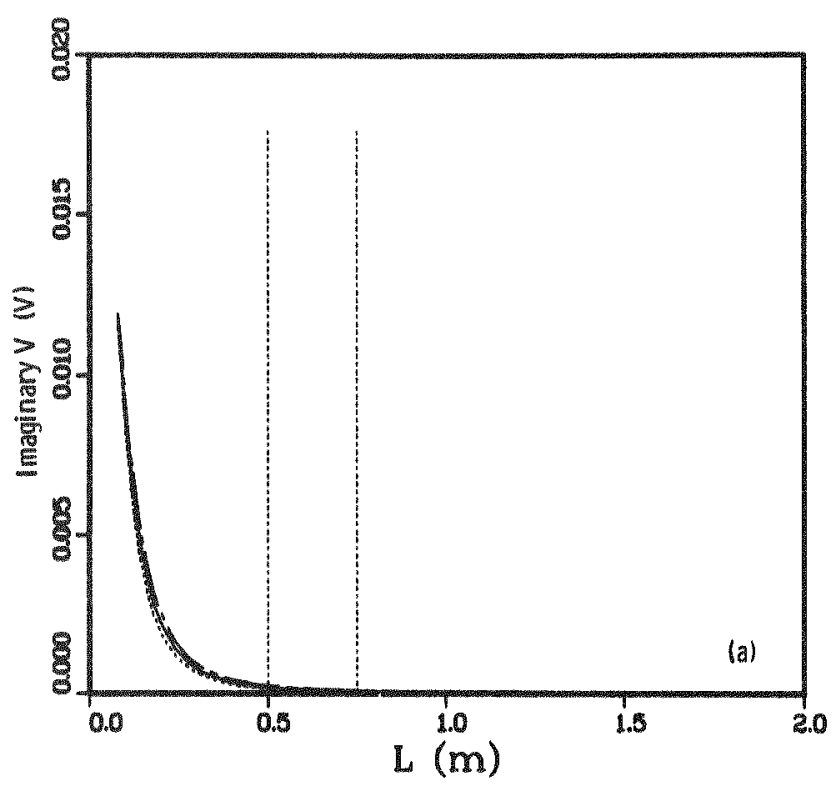

Figure 12. Imaginary Part of Voltage in a Receiver Coil at Axial Distance L From the Dipole Source in Test Problem B

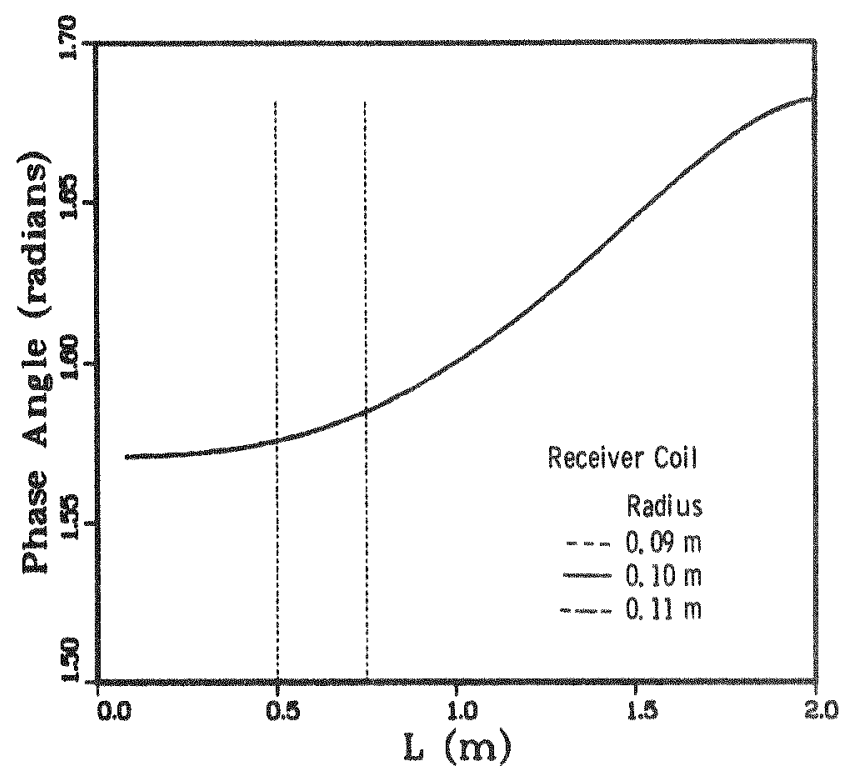

Figure 13. Phase Angle of the Complex Voltage in a Receiver Coil at Axial Distance L From the Dipole Source in Test Problem B 
In a logging operation, analytic results such as those of Moran and Kunz will not be available. Hence the engineer must choose between the two solutions available; i.e., for $\mathrm{DZ}=0.01 \mathrm{~m}, \mathrm{DZSOUR}=0.10 \mathrm{~m}$; and for $\mathrm{DZ}=0.02 \mathrm{~m}$, DZSOUR $=0.20 \mathrm{~m}$. One possible aid in making the choice is the result of a third calculation, perhaps with boundaries even further removed from the source region. That result should confirm that the valid solution has been attained.

\section{Lysne Test Problems and Comparison With Some Available Analytical Results}

As a test of this mathematical approach to solving the equations in a layered medium, several test problems are examined. The electromagnetic parameters are chosen to represent realistic drilling situations with reasonable geologic variables. The earlier Test Problem B suffers from lack of reality for usual logging geometries. Only vertically layered situations are modeled here so that results may be compared directly with analytical results available from the work of $\mathrm{Yu}$ and Reardon. ${ }^{5}$

The source is assumed to be a harmonic magnetic dipole embedded in the earth. The $\mathrm{z}$ axis is vertical and passes through the source. The earth is divided into azimuthally symmetric regions

$$
\begin{array}{ll}
\text { I } & 0 \leq \mathrm{r} \leq 0.0254 \mathrm{~m}, \\
\text { II } & 0.0254<\mathrm{r} \leq 0.11112 \mathrm{~m}, \\
\text { III } & 0.11112<\mathrm{r}<\infty .
\end{array}
$$

Discontinuities in $\epsilon$ and $\sigma$ occur at $\mathrm{r}=2.54 \mathrm{~cm}$ where the sonde meets the borehole fluid and at $\mathrm{r}=$ $0.11112 \mathrm{~m}$ where the fluid meets the rock formation. The problems have several other characteristics in common. The receiver positions of special interest are at vertical distances RECL1 $=0.61 \mathrm{~m}$ and RECL2 $=$ $0.915 \mathrm{~m}$ above the source. The source is a two-turn loop with maximum current $1 \mathrm{~A}$ and radius $0.0254 \mathrm{~m}$. The receiver is a one-turn loop with a small gap for voltage measurement and a radius of $0.0254 \mathrm{~m}$. The sonde is assumed to occupy the $\mathrm{z}$ axis within $0 \leq \mathrm{r} \leq$ $2.54 \mathrm{~cm}$ where $\sigma=0$ and $\epsilon=\epsilon_{\mathrm{o}}$.

\section{Test Problem C: Oil-Drilling Fluid, Frequency $1 \mathrm{MHz}$}

Earth electrical parameters

$\begin{array}{crc}\text { Region } & \epsilon / \epsilon_{\mathrm{O}} & \sigma(\mathrm{mho} / \mathrm{m}) \\ \text { I } & 1.0 & 10^{-10} \\ \text { II } & 2.0 & 10^{-10} \\ \text { III } & 15.0 & 0.00117\left(21 . \omega \epsilon_{\mathrm{o}}\right)\end{array}$

Source frequency $=1 \mathrm{MHz}$

The drilling fluid is oil. A source region within Region I surrounds the source. Fields in the source region are assumed those of a point dipole in a uniform medium. Fields at the boundary of this "source" serve as driving terms for the field distribution in the surrounding earth.

As an example of the computer code use, param-. eters are listed that might change when a new problem is encountered. (Code use is also discussed in Appendix E.)

\section{Possible Changes in IN (Input) Subroutine}
GAMMA $=\gamma 1.0$
DZSOUR
$0.20 \mathrm{~m}$ source extent in $\mathrm{z}$
DRSOUR
$0.024 \mathrm{~m}$ source extent in $\mathrm{r}$

(This value puts the source region

DT

$\mathrm{DZ}$

FREQ

$N Z$

NR

RT entirely within Region I)

RECL1 $0.61 \mathrm{~m}$ vertical source-receiver 0.003 "radial" step size $0.02 \mathrm{~m}$ vertical step size 1.E6 $\mathrm{Hz}$ source frequency 201 number of vertical steps 201 number of radial steps distance 1

RECL2 $0.915 \mathrm{~m}$ vertical source-receiver distance 2

RSET(1) $\quad 0.0254 \mathrm{~m} \mathrm{R}$ for first radial discontinuity

RSET(2) $\quad 0.11112 \mathrm{~m} R$ for second radial discontinuity

TURNS 2 number of turns in source coil

RADIUS $\quad 0.0254 \mathrm{~m}$ radius of source coil and of receiver coil

CURR $1 \mathrm{~A}$ peak current in source coil 


\section{Possible Changes in EP (Electrical Parameter) Subroutine}

The user must assign values of $\epsilon / \epsilon_{\mathrm{o}}$ and $\sigma$ appropriate for the test problem and consistent with the RSET matrix. In the present problem, appropriate $\epsilon$ and $\sigma$ are set in Regions I, II, and III.

\section{Possible Changes in Main Program FIDELE}

$\begin{array}{lll}\text { NITER } & & \begin{array}{l}\text { Number of iterations to } \\ \text { be attempted } \\ \text { Iteration interval be- } \\ \text { Itween printout } \\ \text { Index determining prob- } \\ \text { lem status: }\end{array} \\ & =1 & \begin{array}{l}\text { Start of problem; result } \\ \text { stored on Tape 12 } \\ \text { Problem continued from } \\ \text { Tape 11; result stored on }\end{array} \\ & =2 & \begin{array}{l}\text { Tape 12 } \\ \text { Problem continued from }\end{array} \\ \text { Tape 11, mesh size al- } \\ \text { tered extending solution } \\ \text { to larger distances; result } \\ \text { stored on Tape 12 } \\ \text { Brief printout } \\ \text { Longer printout } \\ \text { Relaxation parameter }\end{array}$

IF(S1.GT.0.2E-19.AND.NI.GT.5) GO TO 360

The $0.2 \mathrm{E}-19$ may be changed to another value to alter the convergence criteria. Another convergence criteria could be substituted.

PETE LYSNE On Line 13 of the code, PROBLEM 4 the comment identifying the problem might be altered.

IF(NI.GT.2450) RP $=2 . /\left(1 .+\operatorname{SQRT}\left(1 .-\mathrm{SPMB}^{* *} 2\right)\right)$ The iteration frequency for altering the relaxation parameter might be altered. The present test of 2450 only allows a change after 2450 iterations. These calculations have not allowed $R P$ to change.
FACT and

$\mathrm{NFACT}=3$

\section{Possible Control Card Changes}

How much calculation time is required? $(\sim 6 \times$ $10^{-5} \times \mathrm{NR} \times \mathrm{NZ}$ s for one iteration)

Do you want to purge a Tape 11 or catalog a new Tape 11 for later plotting or problem continuation?

Do you need to attach a Tape 11 to continue the calculation or to plot results? The tapes are discussed in Appendix E.

Each problem may be started with a few hundred iterations for checking problem input, to verify that convergence is promising, and perhaps to estimate the number of iterations needed for convergence. Then the calculation can be continued (with IPL $=2$ ) for a longer computer run.

Variation of the convergence parameter, S1, is seen in Figure 14. Comparison with Figure 4 suggests convergence is slower than for Test Problem B. Figure 15 illustrates the slow convergence still evident after 2700 iterations. These receiver coils have radii of $0.024 \mathrm{~m}$ even though RADIUS is input at $0.0254 \mathrm{~m}$. With $\mathrm{DT}=0.003$ and $\gamma=1$, a radius of $0.024 \mathrm{~m}$ falls on a mesh point. Interpolation can be used to find the value at $r=$ RADIUS. A basic conflict always remains between the details followed around the source (increasing with decreasing $D T$ and $D Z$ ) and the extent of interference from the outer boundary conditions (that are drawn closer to the source as DT and DZ decrease). The graphical output from the plotting program follows as Figures 16 through 20. Results for three receiver radii are given in Figure 18-19 as an interpolation aid. 


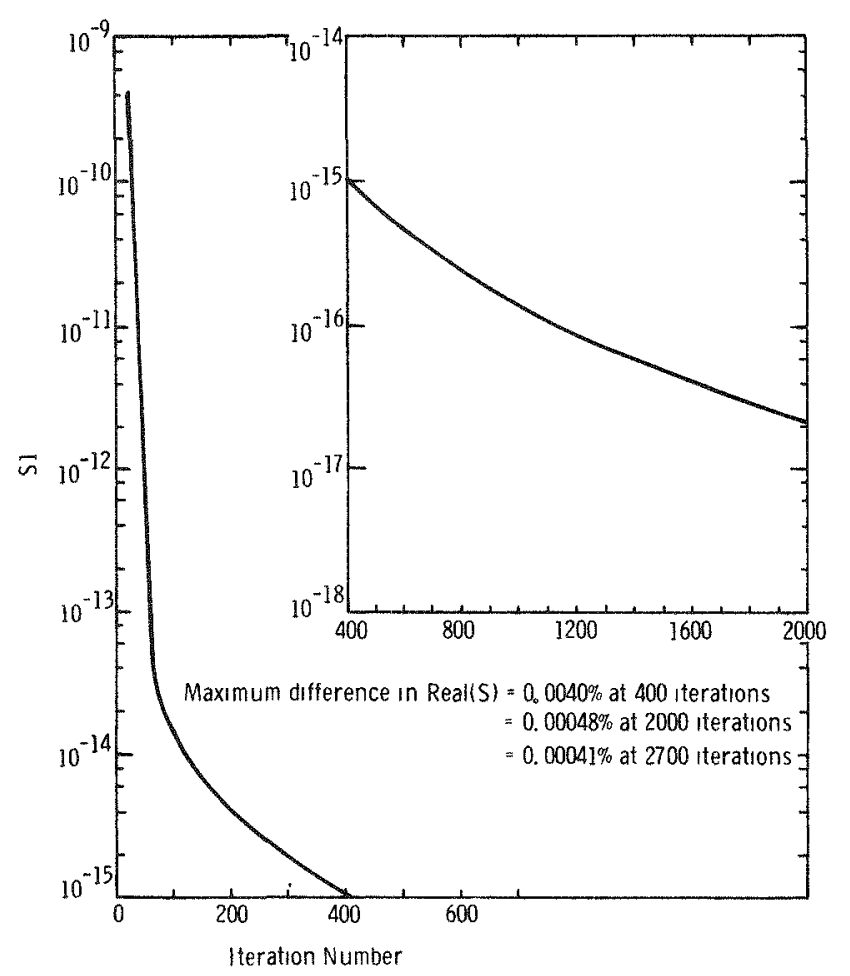

Figure 14. Variation of Convergence Parameter S1 With Iteration Number for Test Problem C

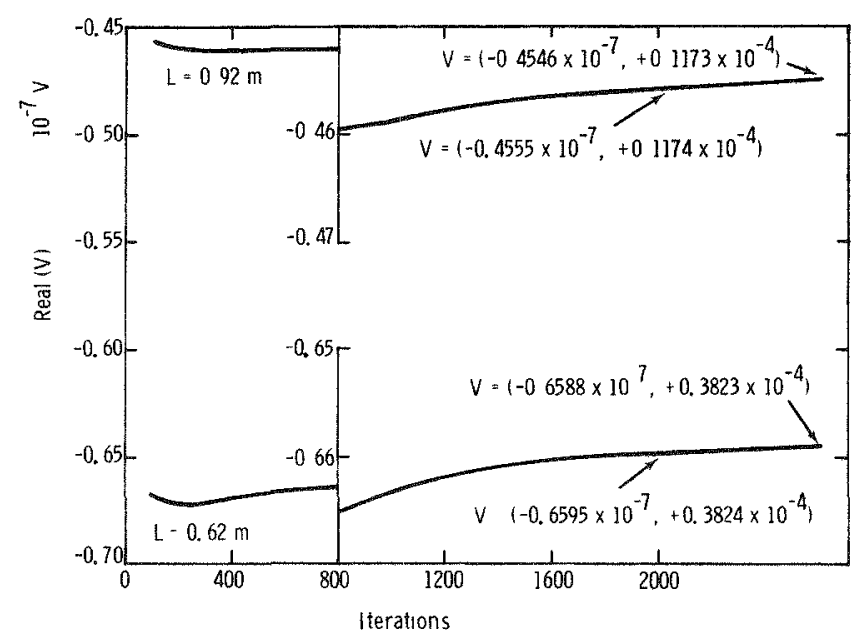

Figure 15. Real Part of the Complex Voltage in Recerver Coils at $\mathrm{L}$ $=062$ and $092 \mathrm{~m}$ for Test Problem C

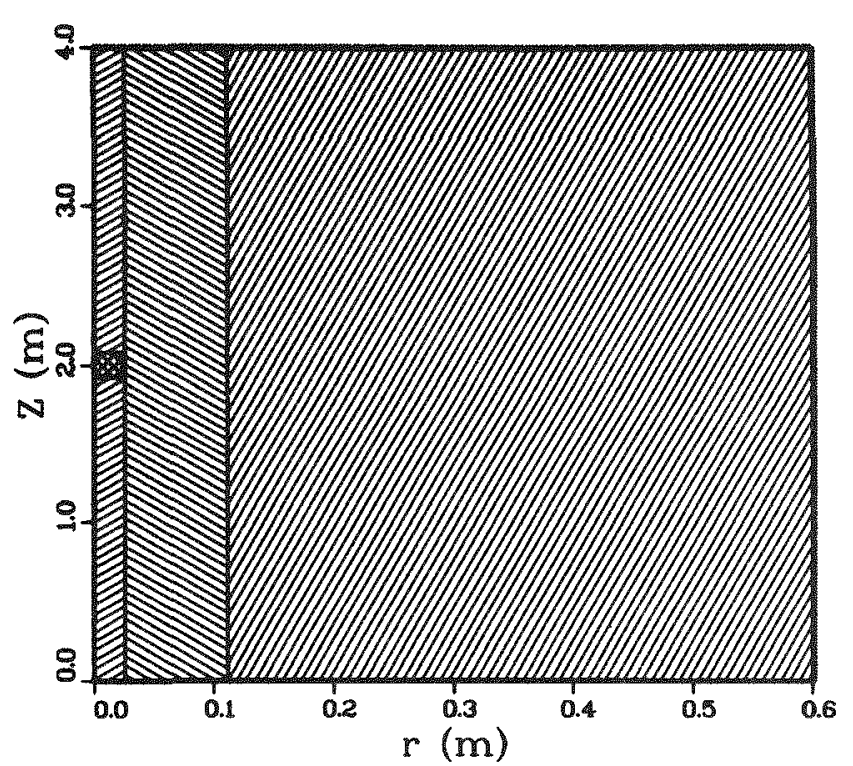

Figure 16. Soll-Layering Geometry for Test Problem C

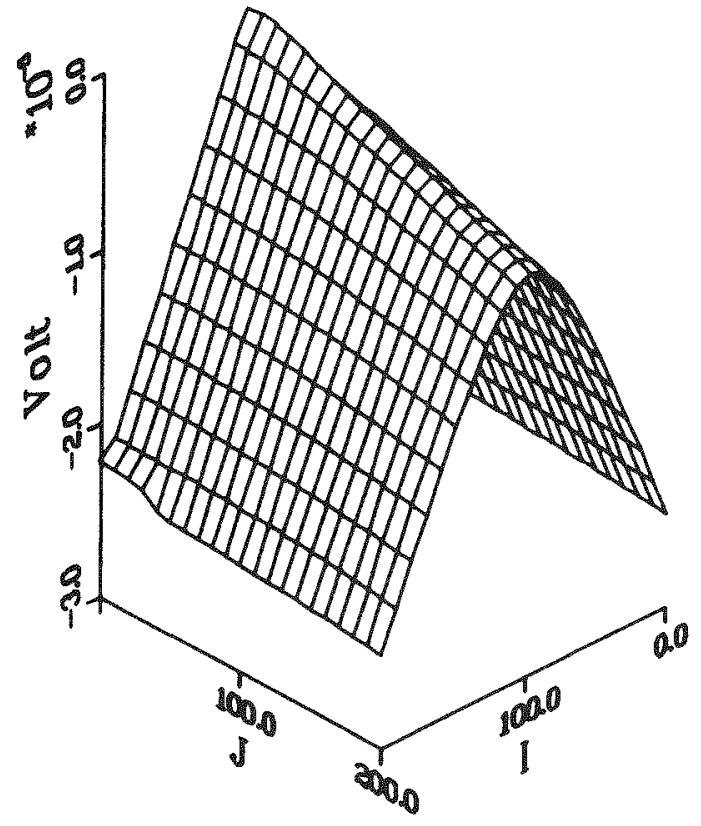

Figure 17. Real $S(\angle, r)$ Distribution for Test Problem $C$ 

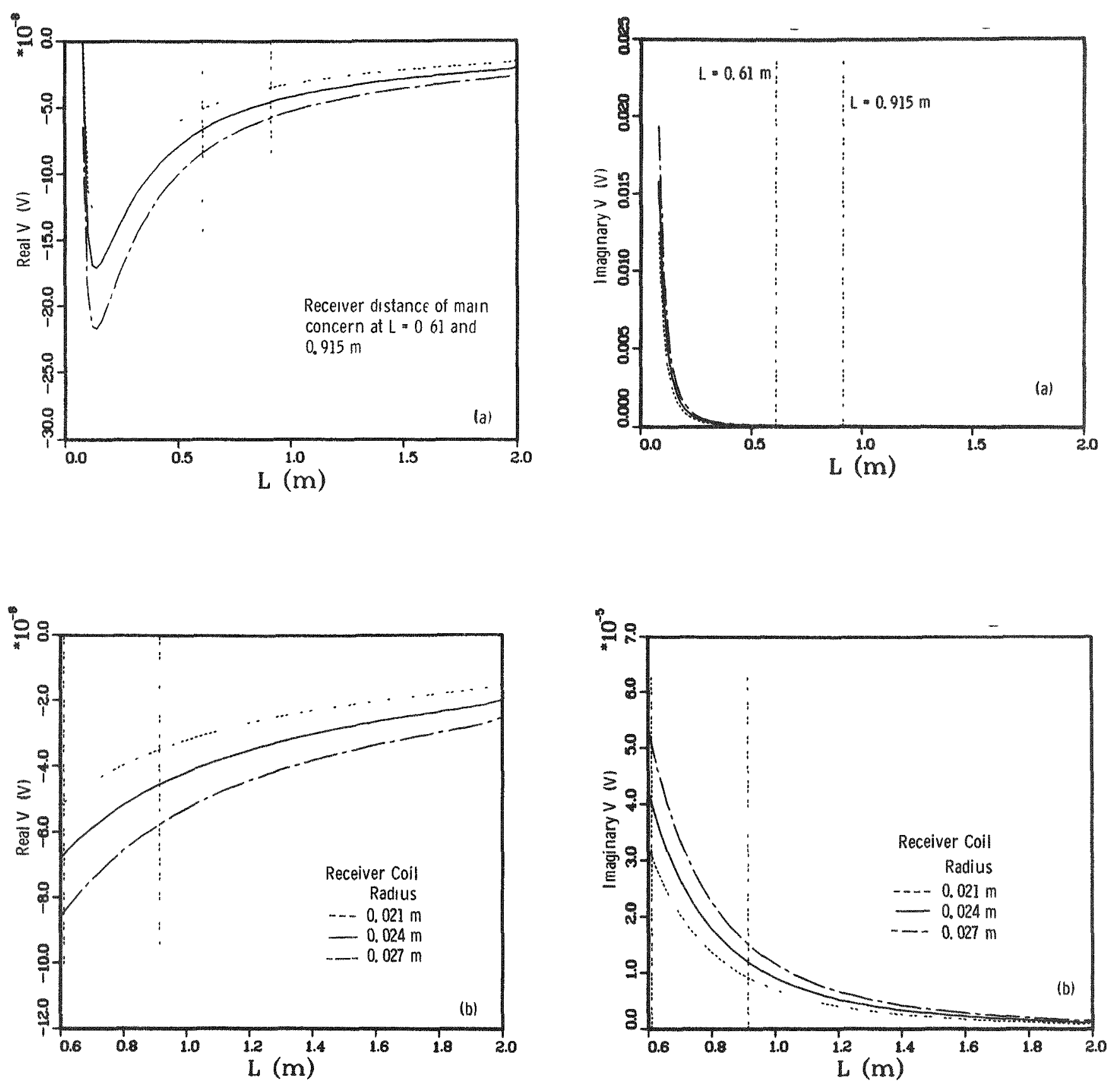

Figure 18. Real Part of Voltage in a Receiver Coll at Axial Distance $L$ From the Dipole Source in Test Problem $C$

Figure 19. Imaginary Part of Voltage in a Recerver Coll at Axal Distance L From the Dipole Source in Test Problem C 


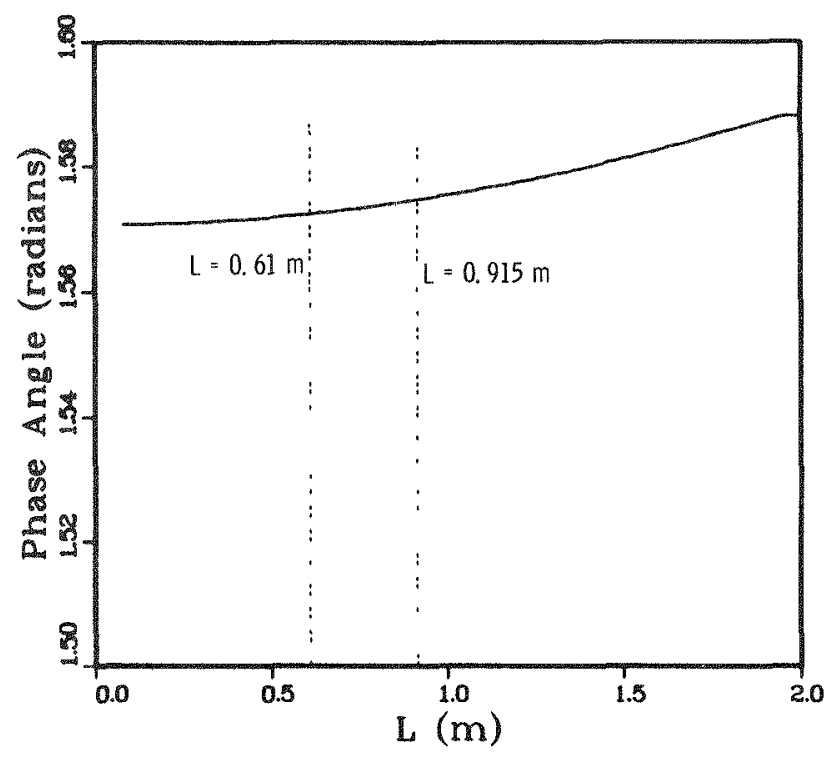

Figure 20. Phase Angle of the Complex Voltage in a Recelver Conl at Axial Distance L From the Dipole Source in Test Problem C (The three curves coincide for coll radı1 $21,2.4$, and $27 \mathrm{~cm}$ )

Sensitivity to some alteration of problem parameters is examined by considering the changes:

Inside the source region

$\epsilon=15 \epsilon_{\mathrm{o}}$ (rather than $\epsilon_{0}$ and 1.E-10)

$\sigma=0.00117 \mathrm{mho} / \mathrm{m}$

Vertical extent of source

DZSOUR $=0.10 \mathrm{~m} \quad$ (rather than $0.2 \mathrm{~m})$

Vertical step size

$$
\mathrm{DZ}=0.01 \mathrm{~m} \quad \text { (rather than } 0.02 \mathrm{~m} \text { ) }
$$

First radial discontinuity

$\operatorname{RSET}(1)=0.0256 \mathrm{~m} \quad($ rather than $0.0254 \mathrm{~m})$

Source and receiver radii

$$
\text { RADIUS }=0.0256 \mathrm{~m}
$$

The more rapid convergence is illustrated in Figures $21-22$ for $\mathrm{S} 1$ and $\operatorname{Real}(\mathrm{V})$ at $\mathrm{L}=0.61$ and $0.91 \mathrm{~m}$. Other graphical results follow (Figures 23 through 27 ). Note the structure resulting from the $\epsilon, \sigma$ discontinuities in the 3-D plot of Real(S) near the peak $($ Real $(S)=0$ ) (Figure 23). The soil-layering diagram (Figure 24) is much the same as Figure 16 except for $z(\max )=2 \mathrm{~m}$ and the differing vertical source extent. The treatment of the source region here introduces some horizontal layering. At receiver distances $\mathrm{L}=$ 0.61 and $0.915 \mathrm{~m}$, little change is apparent in the complex voltages. For example, at receiver radius $0.024 \mathrm{~m}$ and at $\mathrm{L}=0.915 \mathrm{~m}$, Figure 25 indicates Real $\mathrm{V}=-4.6 \times 10^{-8} \mathrm{~V} / \mathrm{m}$ for Modified Problem C. Test Problem $\mathrm{C}$ in Figure 18 yields the same value.

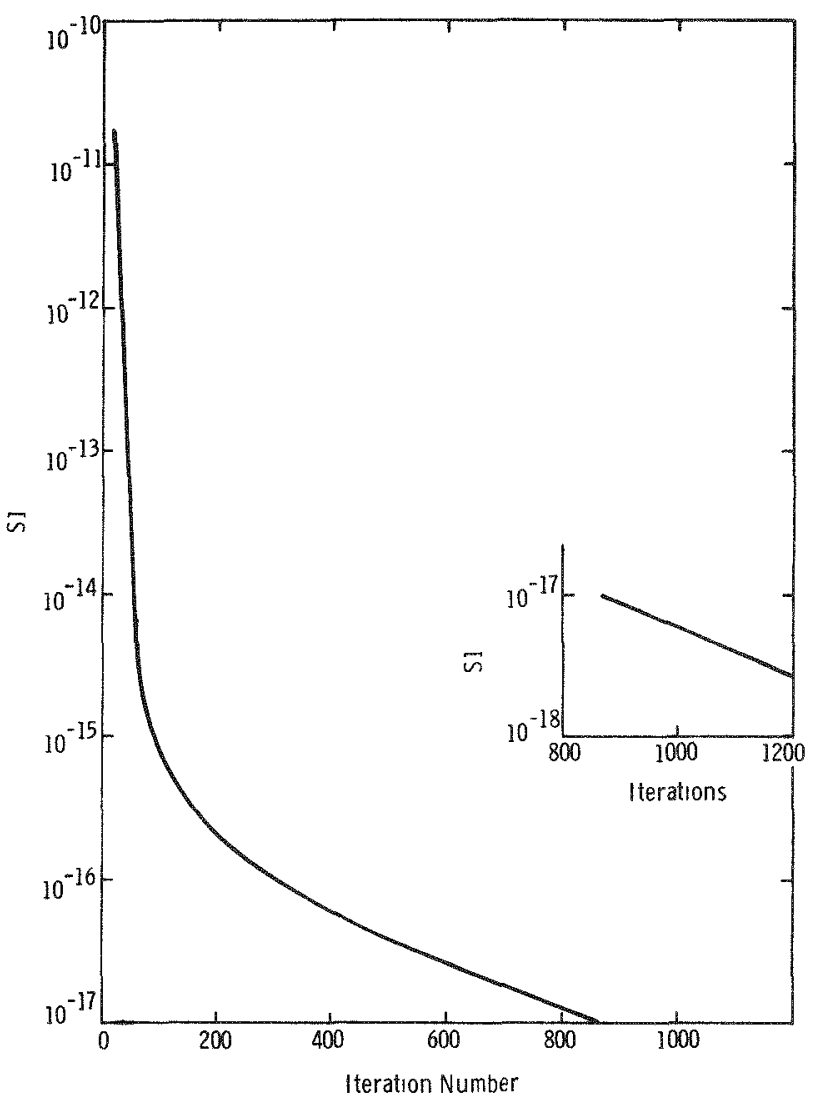

Figure 21. Variation of Convergence Parameter S1 $W_{1}$ th Iteration Number for Modified Test Problem C

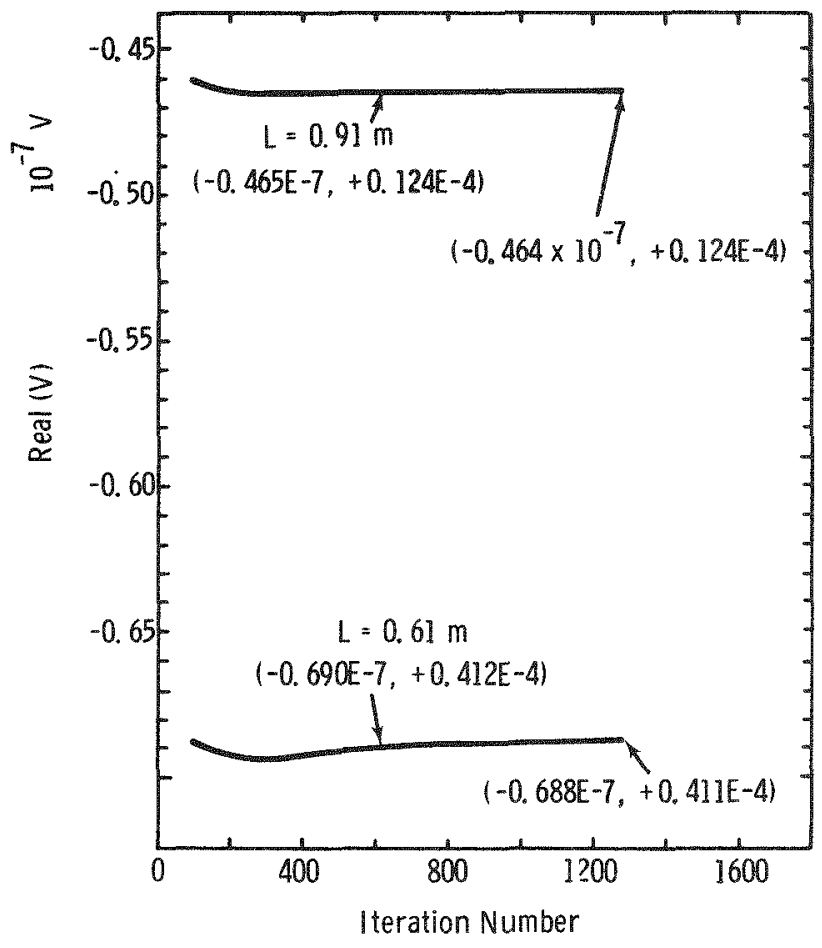

Figure 22. Real Part of the Complex Voltage in Receiver Conls at $L$ $=061$ and $091 \mathrm{~m}$ for Modified Test Problem C (Recerver radius is $24 \mathrm{~cm}$ ) 


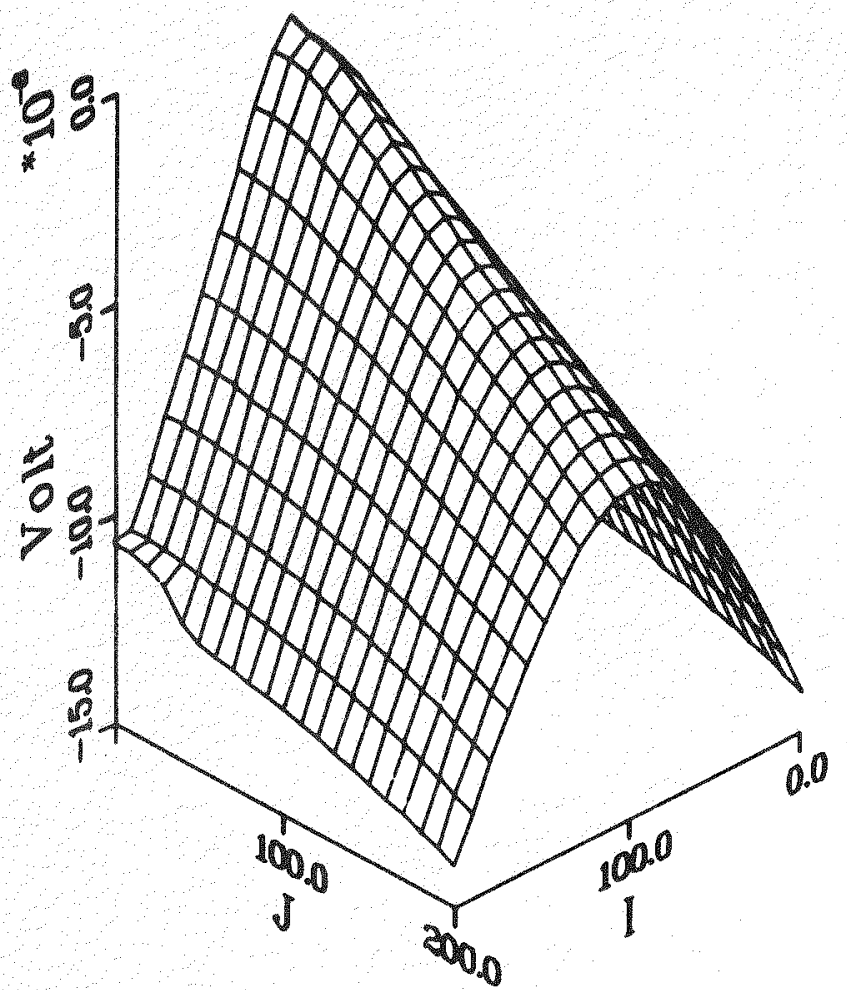

Figure 23. Real S( $\mathrm{z}, \mathrm{r})$ Distribution for Modified Test Problem C

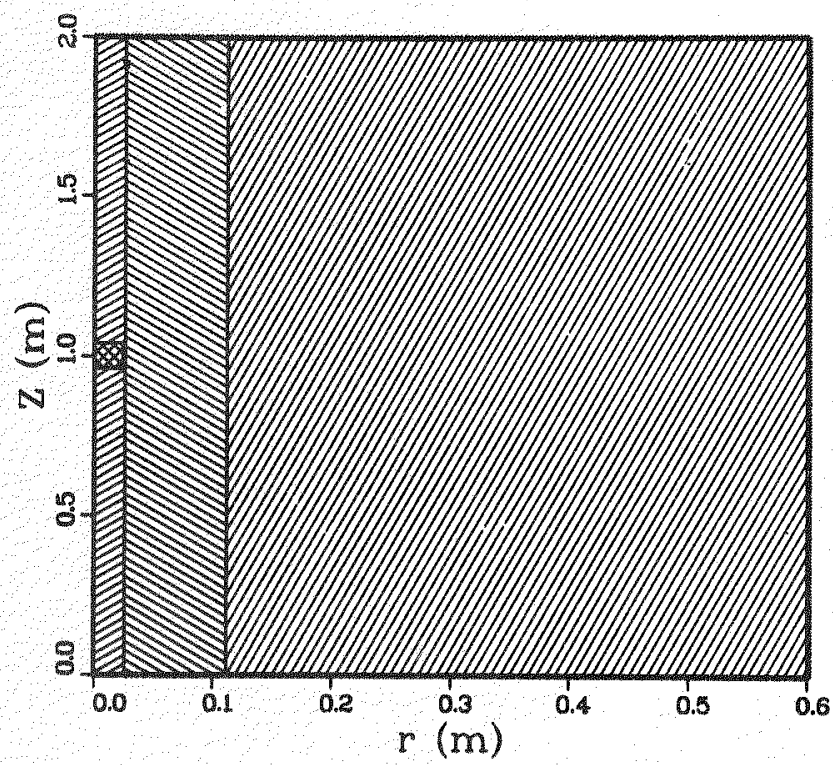

Figure 24. Soil-Layering Geometry for Modified Test Problem C
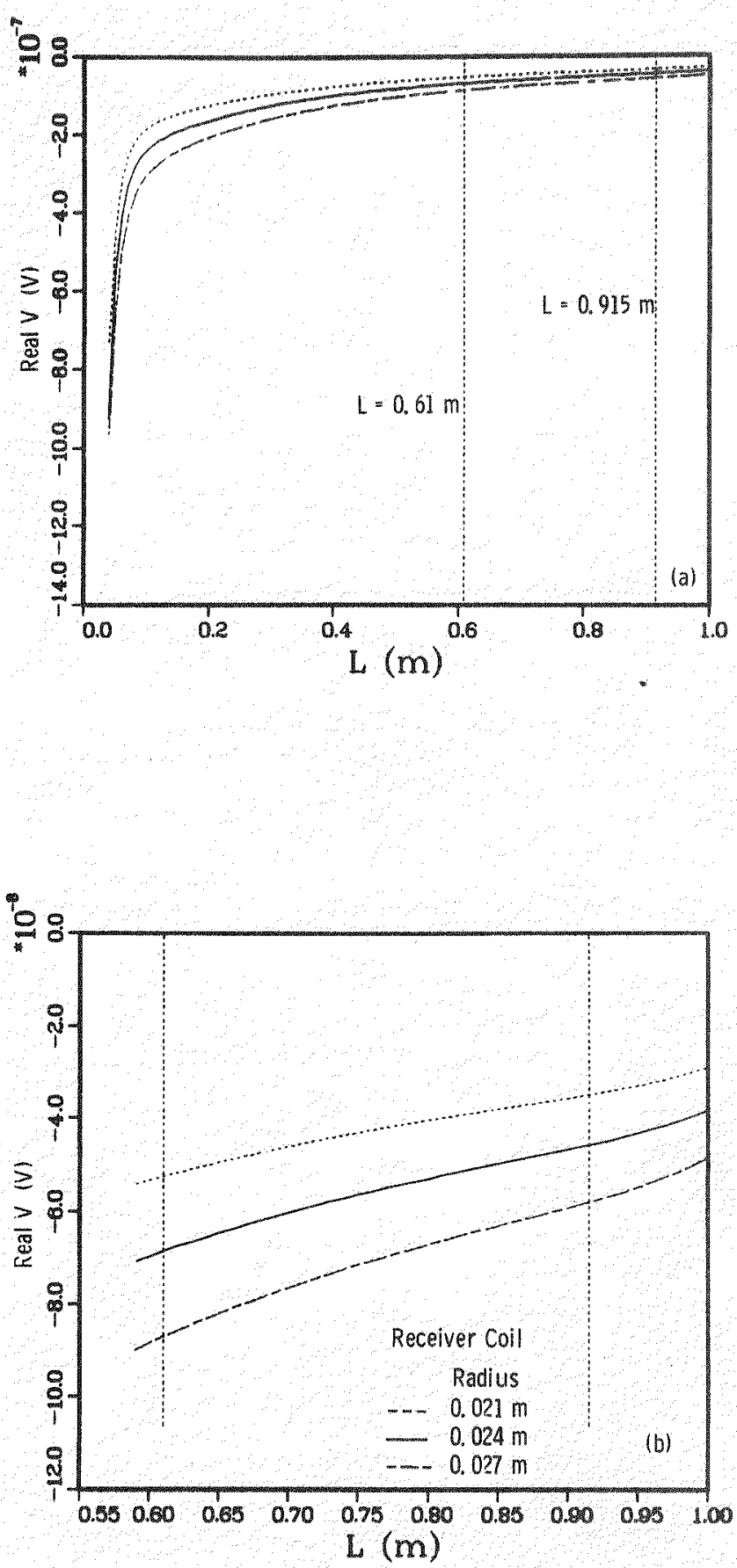

Figure 25. Real Part of Voltage in a Receiver Coil at Axial Distance L From the Dipole Source in Modified Test Problem C 

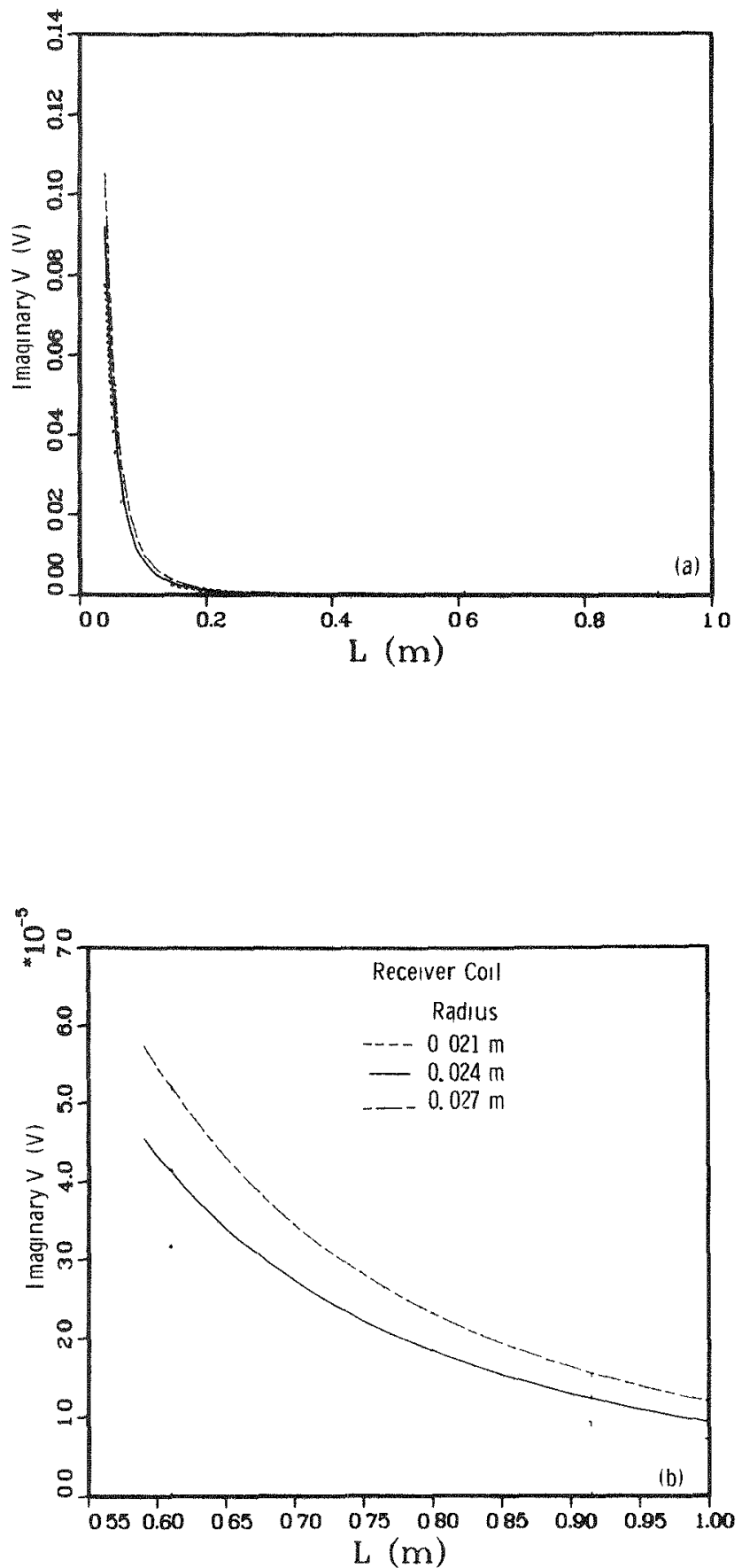

Figure 26. Imaginary Part of Voltage in a Receiver Coll at Axial Distance L From the Dipole Source in Modified Test Problem C

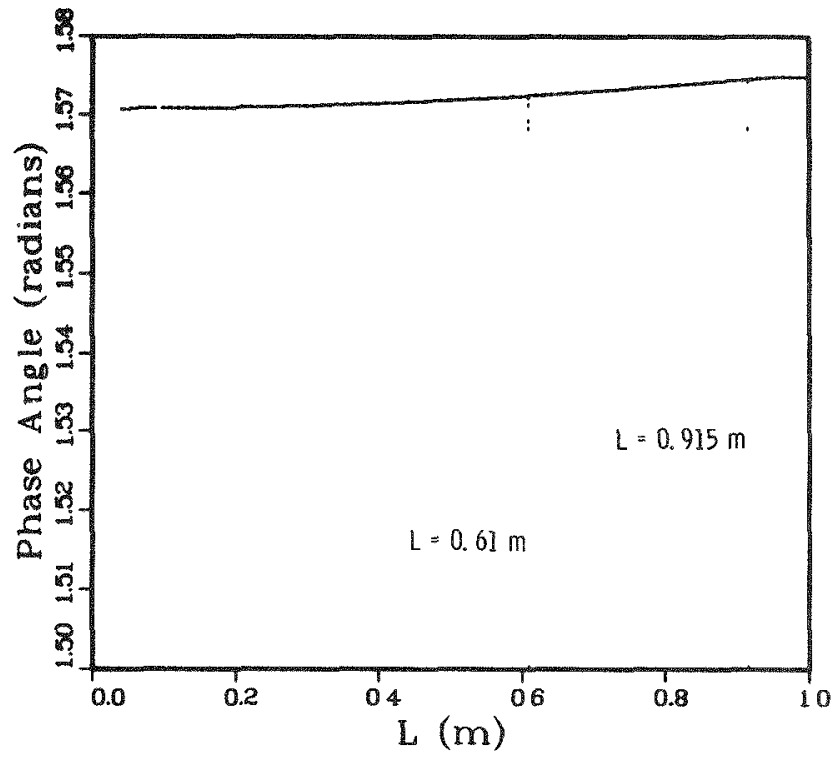

Figure 27. Phase Angle of the Complex Voltage in a Recelver Conl at Axial Distance L From the Dipole Source in Modified Test Problem $C$ (The curves coincide for the three receiver radin considered)

\section{Test Problem D: Saltwater Drilling Fluid, Frequency $1 \mathrm{MHz}$}

Earth electrical parameters

$$
\begin{array}{crll}
\text { Region } & \epsilon / \epsilon_{\mathbf{O}} & & \sigma(\mathrm{mho} / \mathrm{m}) \\
\text { I } & 1.0 & 1 . \mathrm{E}-10 \\
\text { II } & 800 & 2.5 \\
\text { III } & 36.4 & 0.0566\left(10^{3} \omega \epsilon_{\mathrm{o}}\right)
\end{array}
$$

All other parameters are as for Test Problem C. Here the drilling fluid is water with a reasonably high salt content. On Line 13 of the code the comment card is altered to identify the current problem. Results are given in Figures 28 through 33. The soillayering geometry (not shown) is identical to that in Figure 16.

In Problem D the spatial region of the source extends $0.20 \mathrm{~m}$ vertically and $0.024 \mathrm{~m}$ radially. Hence, at the vertical extremities, the source region is $0.1 \mathrm{~m}$ from the point dipole; i.e., four times farther than the 
radial extent of the source region. The radial extent is short enough to restrict the source region to within Region I of the layered earth. The vertical extent is chosen somewhat arbitrarily; short compared to the vertical distances for receivers, yet long enough that the rapid variation of $S$ near the source can be followed by the finite-difference solution scheme.

Let us consider Problem D with DZSOUR altered to $0.1 \mathrm{~m}$ and $\mathrm{DZ}=0.01 \mathrm{~m}$ in order to follow the variation more closely near the source (but bringing the boundary nearer receiver positions). RSET(1) and RADIUS have also been altered to $0.0256 \mathrm{~m}$. In this smaller spatial region convergence occurs slightly more rapidly. After 2400 iterations, $\mathrm{S} 1=8.84 \times 10^{-18}$; voltages become $(-0.7292 \mathrm{E}-5,+0.3804 \mathrm{E}-4)$ and $(-0.3131 \mathrm{E}-5,+0.1101 \mathrm{E}-4)$ at $\mathrm{L}=0.62$ and $0.92 \mathrm{~m}$. These voltages are within $\sim 5 \%$ of the earlier values.

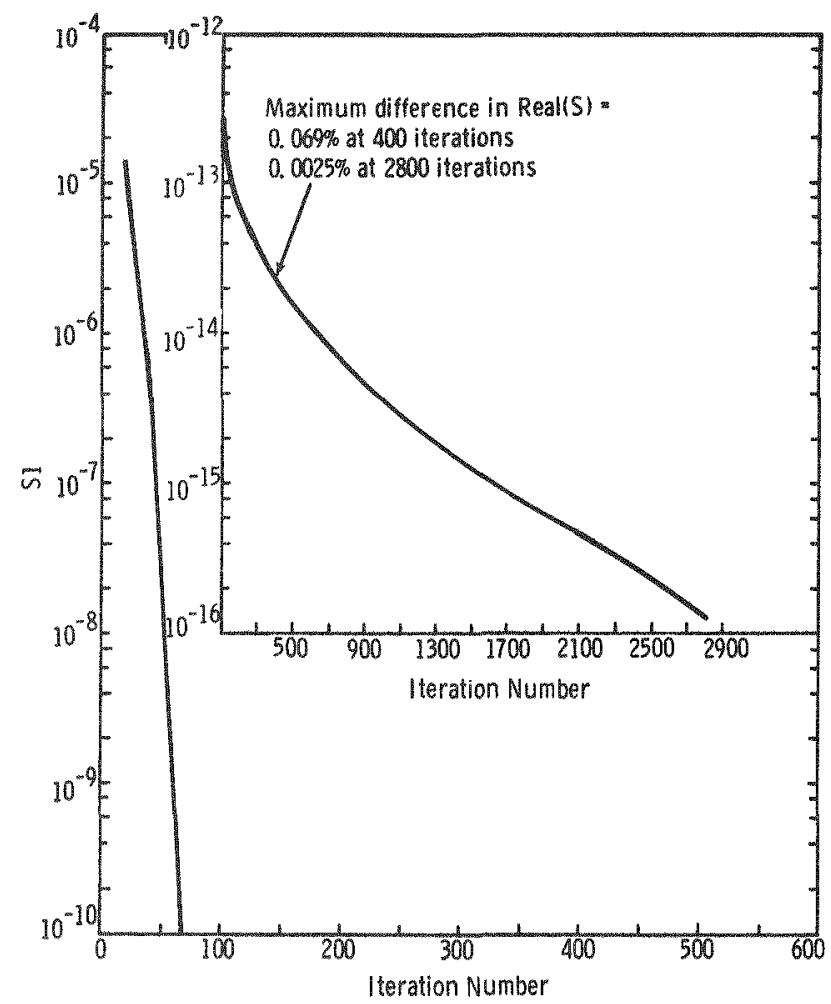

Figure 28. Variation of Convergence Parameter $\$ 1$ With Iteration Number for Test Problem D

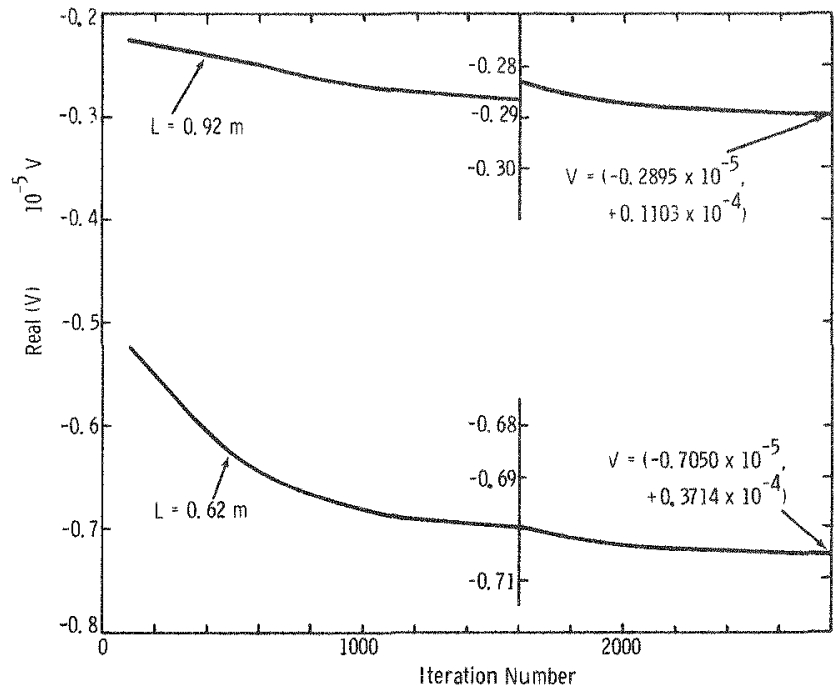

Figure 29. Real Part of the Complex Voltage in Receiver Coils at $L$ $=0.62$ and $0.92 \mathrm{~m}$ (Coil radius is $2.4 \mathrm{~cm}$ )

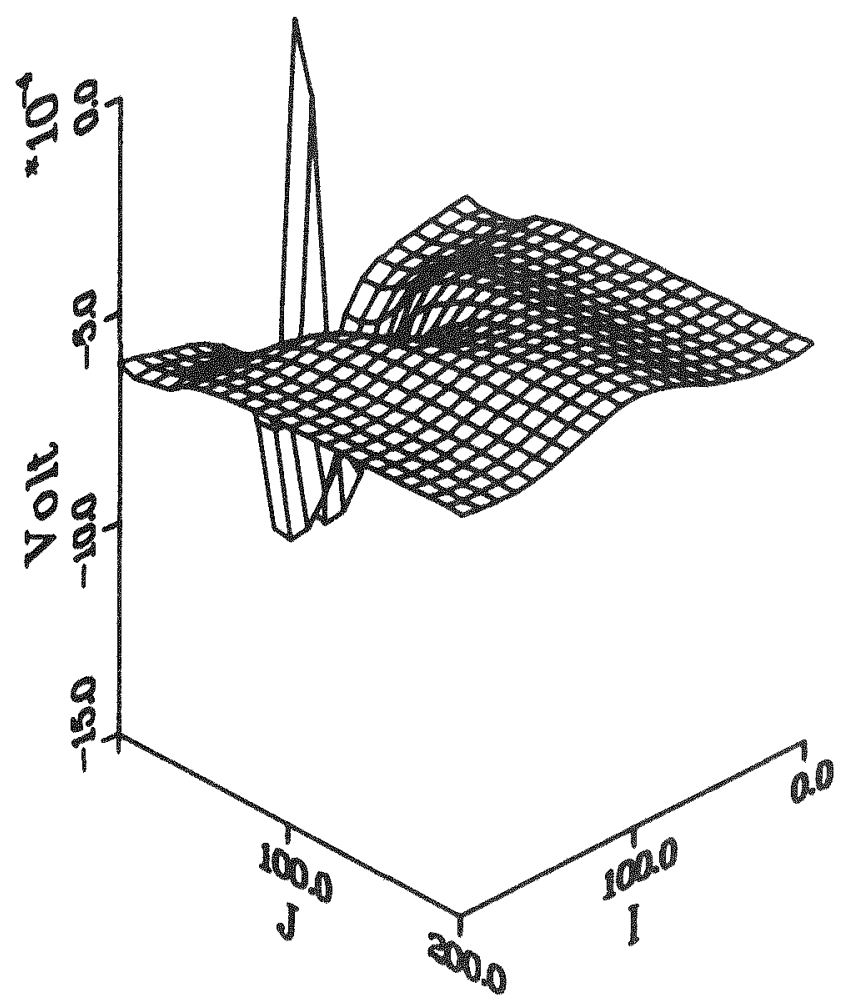

Figure 30. Real $S(z, r)$ Distribution for Test Problem D 

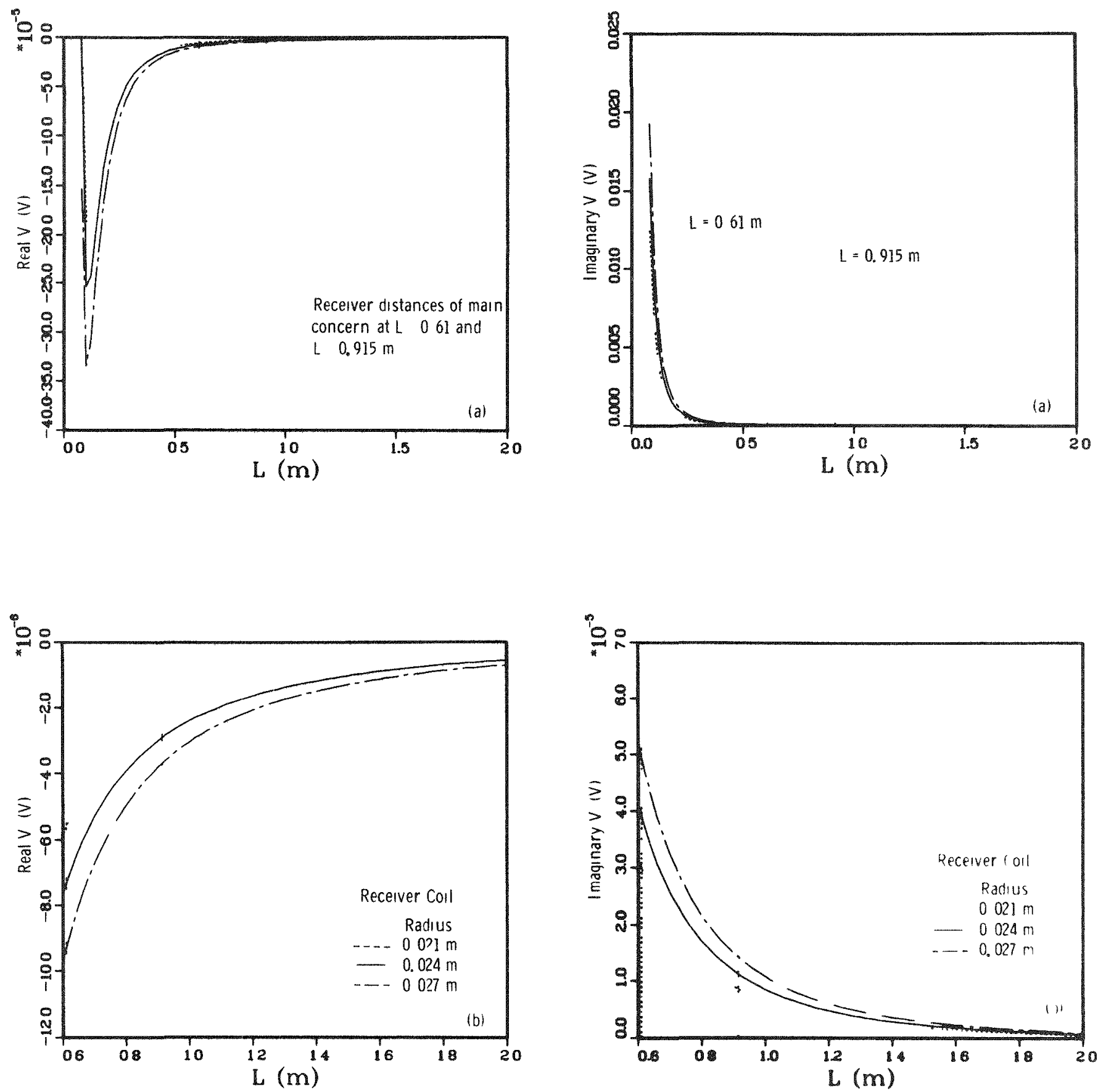

Figure 31. Real Part of the Complex Voltage in a Receiver Coll at Axial Distance L From the Dipole Source in Test Problem D

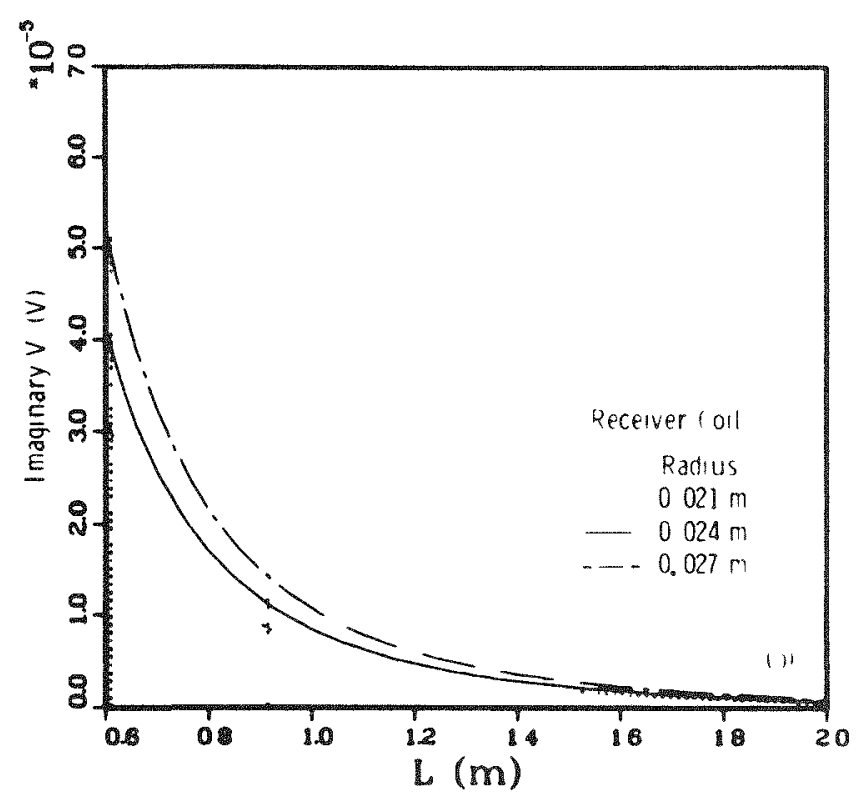

Figure 32. Imaginary Part of the Complex Voltage in a Receiver Coll at Axial Distance L From the Dipole Source in Test Problem D 


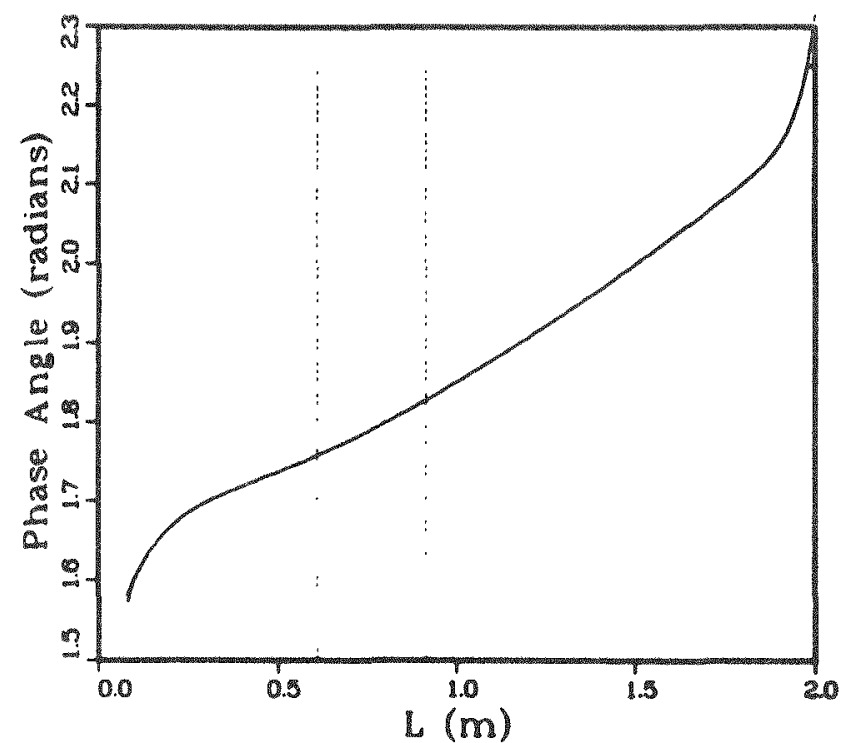

Figure 33. Phase Angle of the Complex Voltage in a Receiver Coil at Axial Distance L From the Dipole Source in Test Problem D (The three curves coincide for receiver coil radii $2.1,2.4$, and $2.7 \mathrm{~cm}$ )

Differences in the input on early attempts at solving Problem D allow us to examine the sensitivity to alteration of some problem parameters. Consider the changes:

Inside the source region

$$
\begin{aligned}
& \epsilon=36.4 \epsilon_{\mathrm{o}} \\
& \sigma=0.0556 \mathrm{mho} / \mathrm{m} \\
& \text { DRSOUR }=0.10 \mathrm{~m} \\
& \mathrm{DZSOUR}=0.20 \mathrm{~m} \\
& \mathrm{DZ}=0.01 \mathrm{~m} \\
& \mathrm{DT}=0.00714142 \\
& \text { GAMMA }=1.1 \\
& \mathrm{NR}=201 \\
& \mathrm{NZ}=325 \\
& \mathrm{RSET}(1)=0.0256 \mathrm{~m} \\
& \text { RADIUS }=0.0256 \mathrm{~m}
\end{aligned}
$$

Radial extent of source

Vertical step size $\gamma$ parameter

Results for this Modified Test Problem $D$ are given in Figures 34 through 41 .

The choice of DT may appear odd. With $\gamma=1.1$ this $D T$ sets $R=0.02559 \mathrm{~m}$ at $J=6$ and $R=0.11112$ $m$ at $J=20$. The other choices introduce horizontal layering around the source and allow the source region to span portions of Regions I and II.
The product $N R * N Z$ is limited by convenient computer storage to $\$ 325 * 201$. Computer time required is proportional to this product. Small values of NR and NZ limit the spatial extent of the solution. When NR or NZ is too small, the problem must be solved in stages, with the source region expanded at each stage. Fortunately, for Test Problem D and for this Modified Test D, such extensions are not necessary. The extensions are tested in Appendix D.

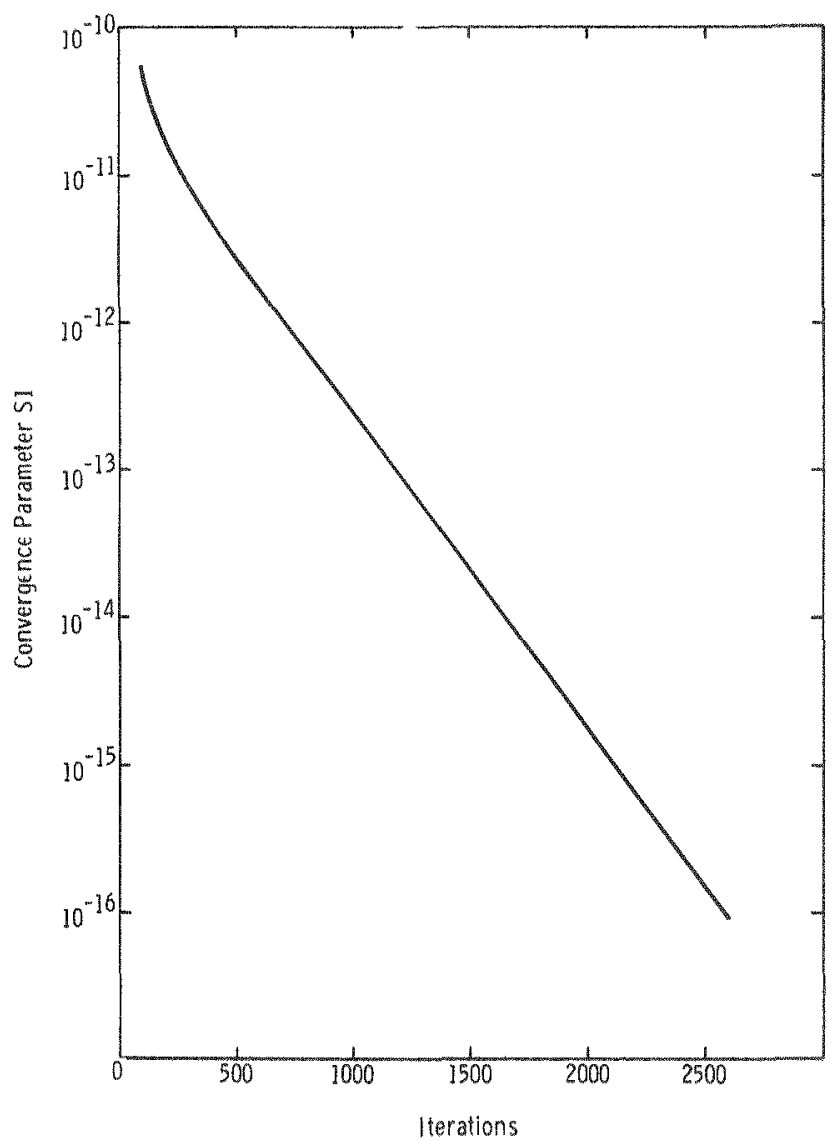

Figure 34. Variation of Convergence Parameter S1 With Iteration Number for Modified Test Problem D 

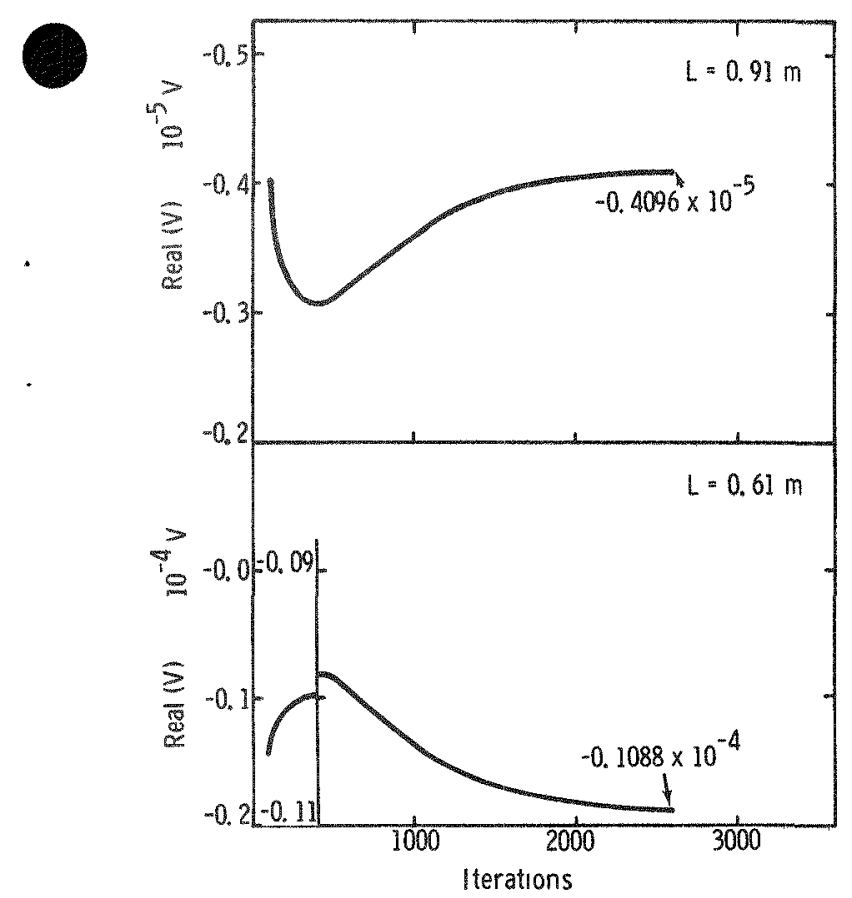

Figure 35. Real Part of the Complex Voltage in Receiver Colls at $\mathrm{L}$ $=061$ and $091 \mathrm{~m}$ for Modified Test Problem D (Corl radius is 256 $\mathrm{cm})$

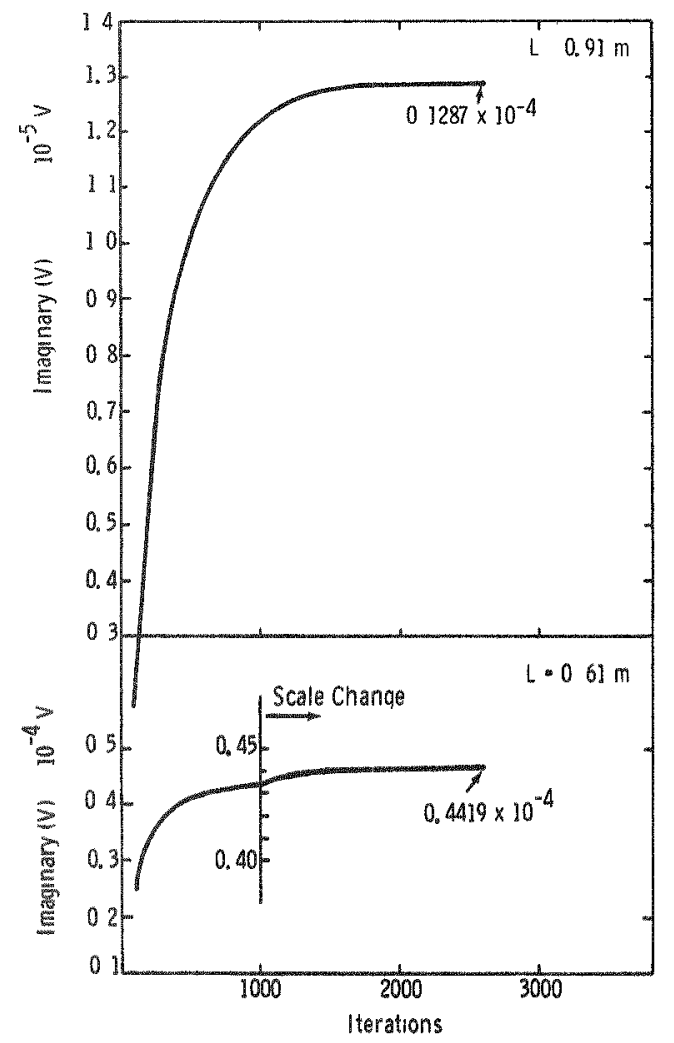

kigure 36. Imaginary Part of the Complex Voltage in Recenver Cols at $L=061$ and $091 \mathrm{~m}$ for Modified Test Problem $D$

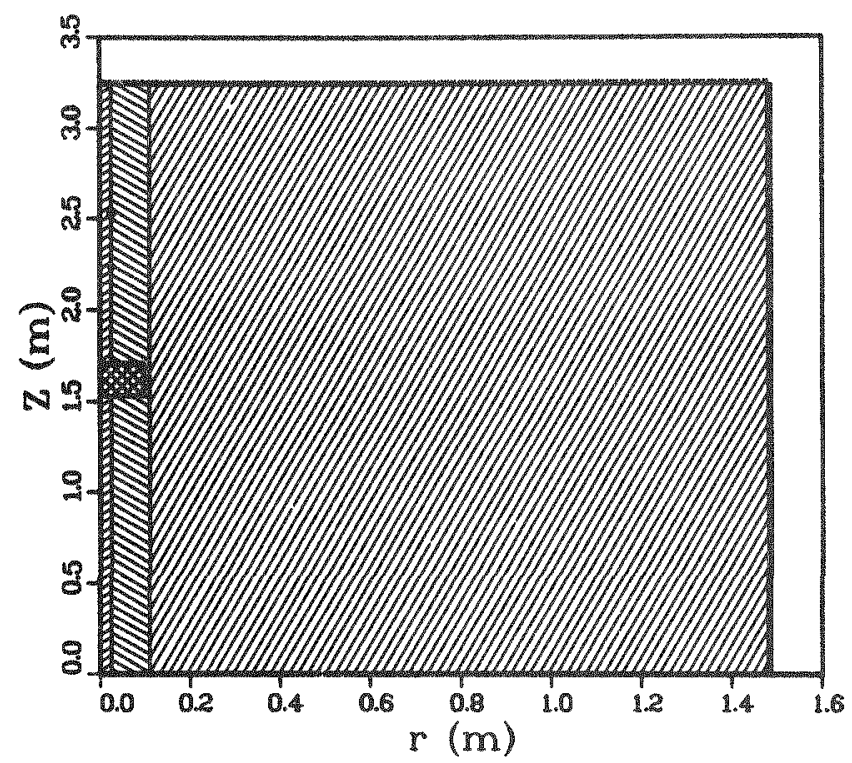

Figure 37. Soll-Layerng Geometry for Modified Test Problem D

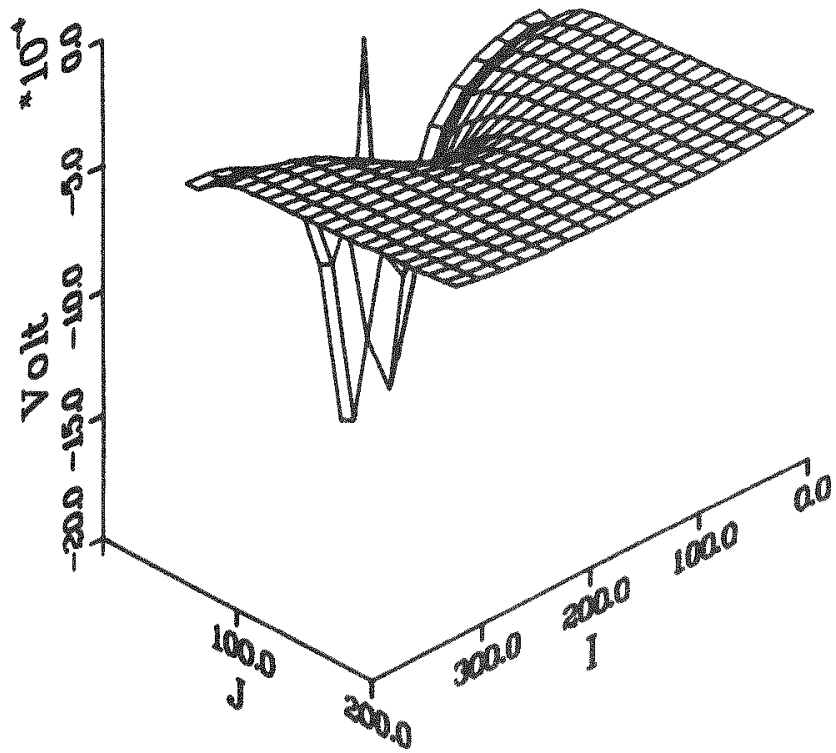

Figure 38. Real S( $\left.\alpha_{r} r\right)$ Distributron for Modrfed Test Problem D 

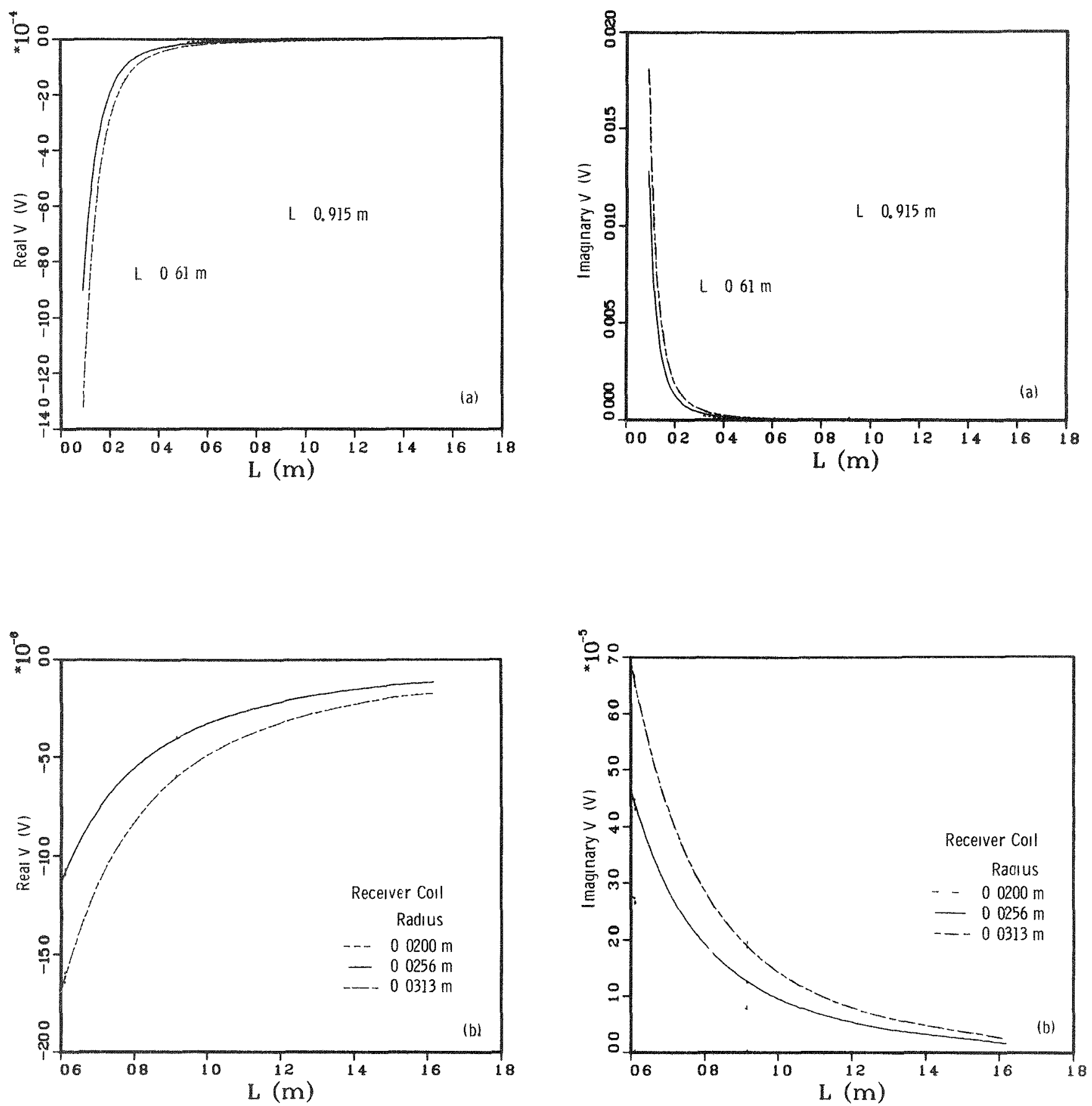

Figure 39 Real Part of Voltage in a Recenver Coil at Axial Distance $L$ From the Dipole Source in Modified Test Problem D

Figure 40. Imaginary Part of Voltage in a Receiver Coil at Axial Distance L From the Dipole Source in Modified Test Problem D 


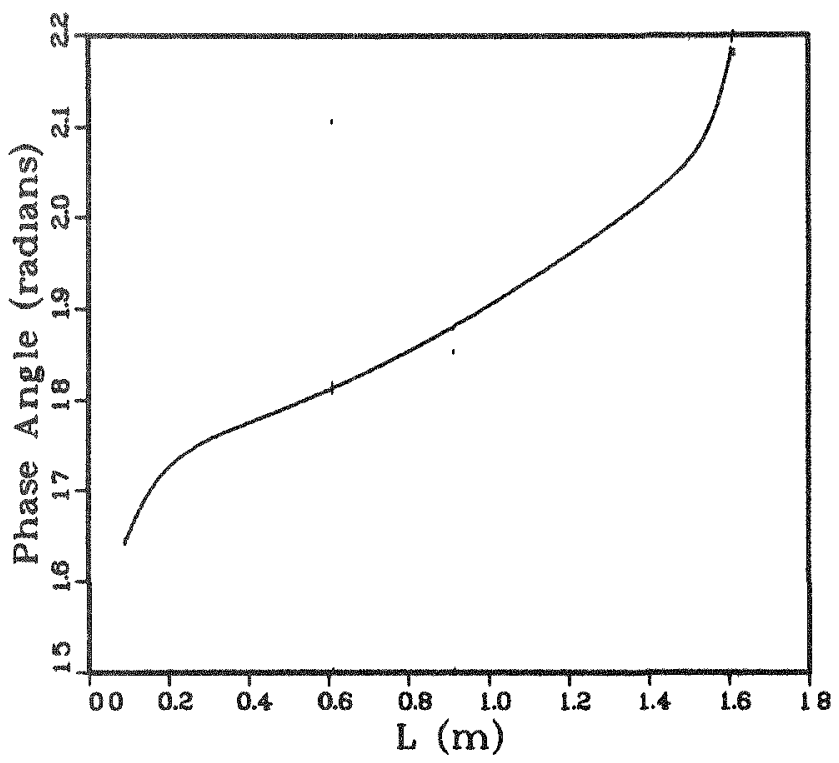

Figure 41. Phase Angle of the Complex Voltage in a Recetver Coll at Axial Distance L From the Dipole Source in Modified Test Problem D (The three curves concide for recerver coll radn 20 , 256 and $313 \mathrm{~cm}$ )

\section{Test Problem E: Saltwater Drilling Fluid, Frequency $30 \mathrm{MHz}$}

This test problem is introduced by enumerating the changes used in converting from Test Problem $\mathrm{C}$ to Test Problem E. The EP subroutine is altered to give the following earth electrical parameters:

$\begin{array}{crl}\text { Region } & \epsilon / \epsilon_{0} & \sigma(\mathrm{mho} / \mathrm{m}) \\ \text { I } & 1.0 & 10^{-10} \\ \text { II } & 80.0 & 2.5 \\ \text { III } & 16.6 & 0.075\left(45 \omega \epsilon_{\mathrm{o}}\right)\end{array}$

Here water is the drilling fluid (Region II). The source frequency is altered to $30 \mathrm{MHz}$. The comment card on Line 13 of the code is altered to identify the problem.

Convergence occurs more slowly than in previous problems. The convergence parameter S1 decays much less rapidly with iteration number (Figure 42). As an indication of how the voltage is altered by the iterations, we also furnish Figure 43 for $\operatorname{Re}(V)$ at $L=$ $092 \mathrm{~m}$. The oscillations result from the increase in frequency, coupled with the poor initial guess for $S(I, I)$. If several such oscillations occur inside the spatual mesh, the oscillations may grow, giving a divergent "solution." Smaller DT, DZ or larger
DZSOUR, DRSOUR should then improve convergence. However, the DT and $\mathrm{DZ}$ parameters cannot be reduced without limit Numerical noise can also cause divergence.

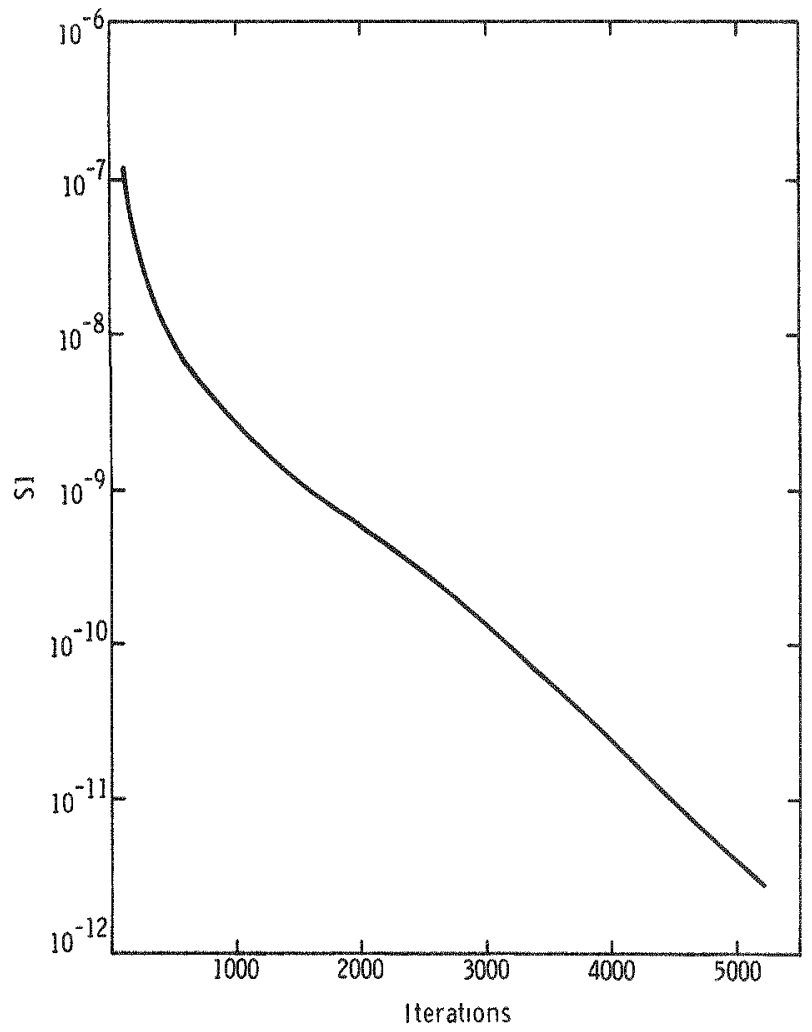

Figure 42. Variation of Convergence Parameter $S 1$ With Iteration Number for Test Problem E

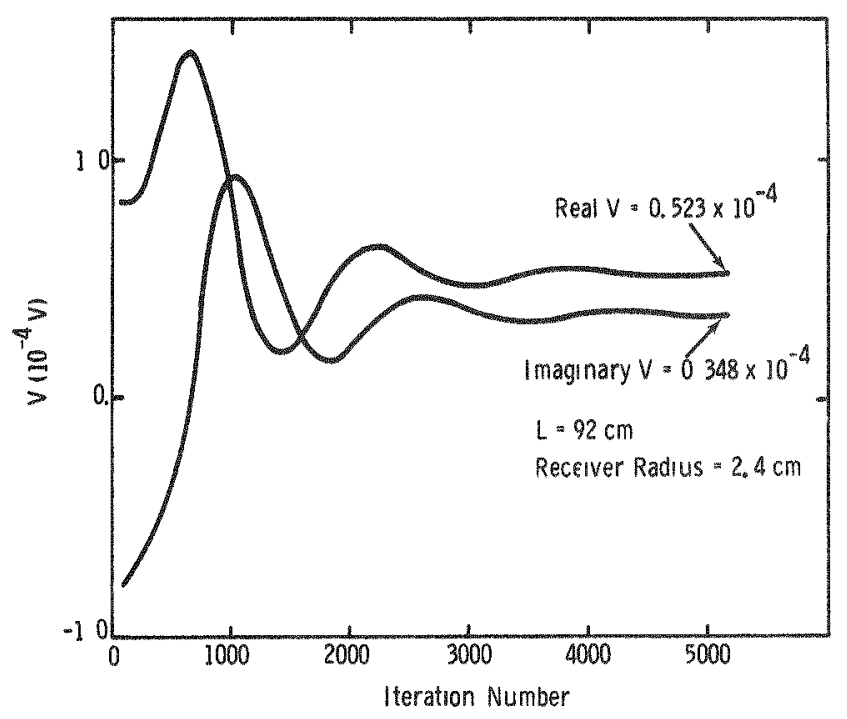

Figure 43. Convergence of the Real and Imaginary Parts of the Complex Voltage on a Recerver Coll for Test Problem E 
After 5200 iterations the $\mathrm{S} 1$ has fallen four orders of magnitude, and the oscillations at $\mathrm{L}=0.92 \mathrm{~m}$ have essentially damped out. Indications are that convergence has been attained. Examination of the maximum percent difference in Real (S) between iterations suggests otherwise. At 5200 iterations the maximum difference is $628 \%$. It occurs at $\mathrm{I}=175, \mathrm{~J}=$ 191. During the preceding few hundred iterations, these differences are of the same order of magnitude. It is expected that the differences occur where Real(S) passes through zero. Since previous test problems did not encounter an oscillating Real(S), they were not faced with the problem. Printout indicated the value $S(175,191)=\left(0.68 \times 10^{-6}, 0.35 \times 10^{-2}\right)$ was indeed passing through a position where $\operatorname{Real}(S)=0$. Because of this possibility, the test of maximum percent difference in Real(S) might be replaced by the maximum percent difference in $|S|$ or $|S|^{2}$. However, such a change requires an additional complex multiplication for each matrix element at each iteration. The difference in Real(S) is calculated as part of the iterative process so that comparison involves little overhead. Perhaps in some applications the $|S|^{2}$ test may be desired.

We could also replace the Real(S) difference test with a more complicated test when Real(S) $<<$ Imaginary(S). At present we use the maximum percent difference in Real(S) for information, not as a criterion for convergence.

The soil-layering diagram for Test Problem $E$ is identical to Figure 16. The other graphical output is given in Figures $44-47$.

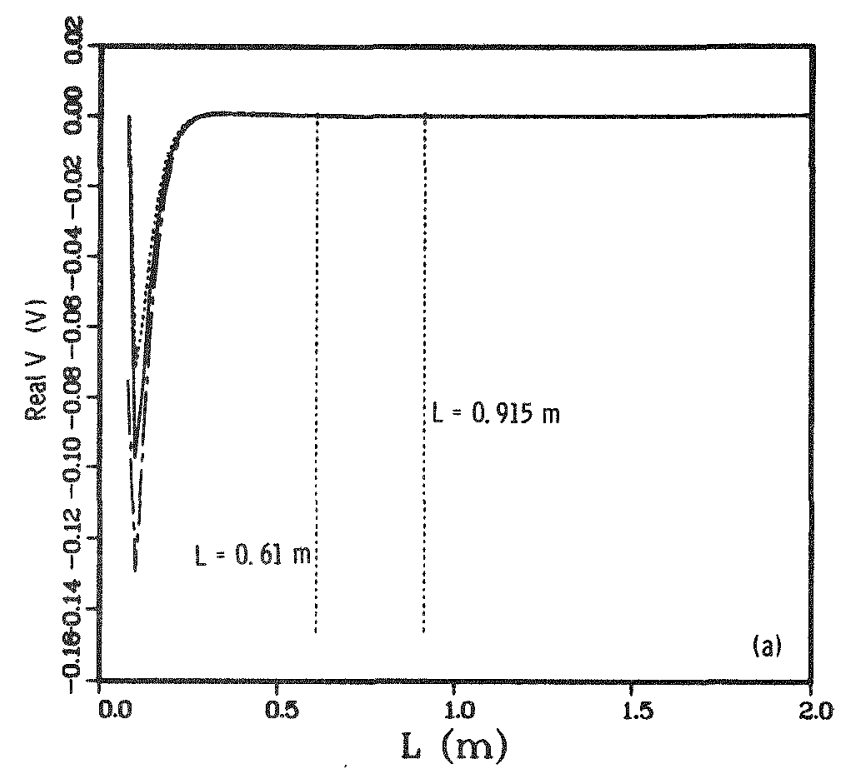

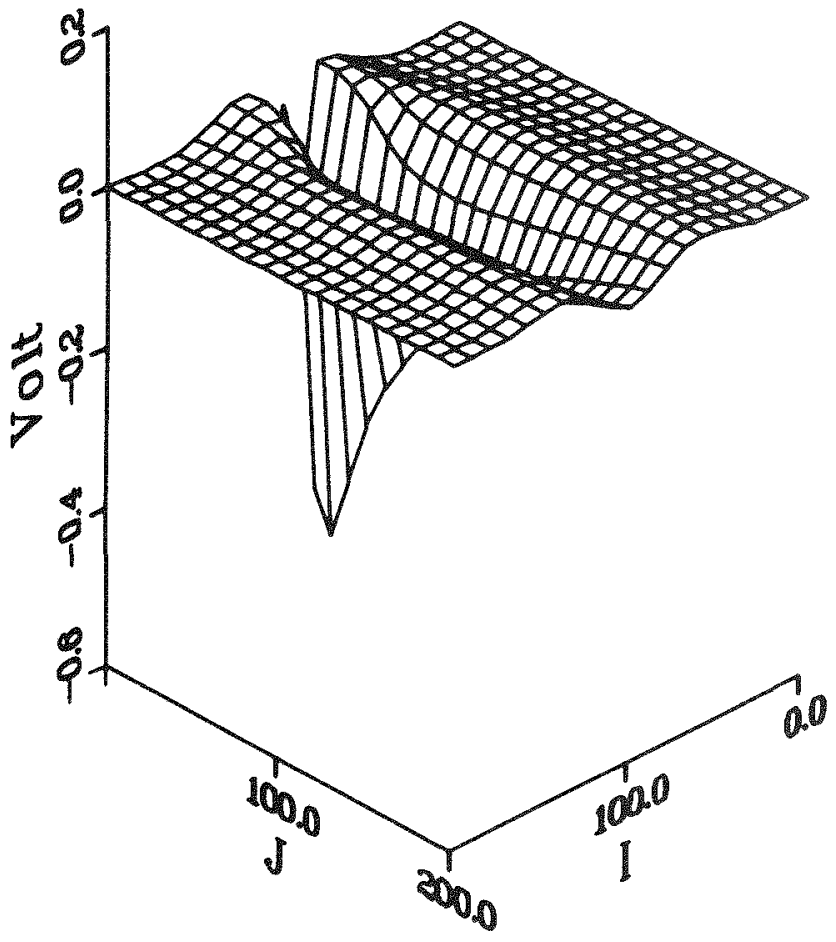

Figure 44. Real S(z,r) Distribution for Test Problem E

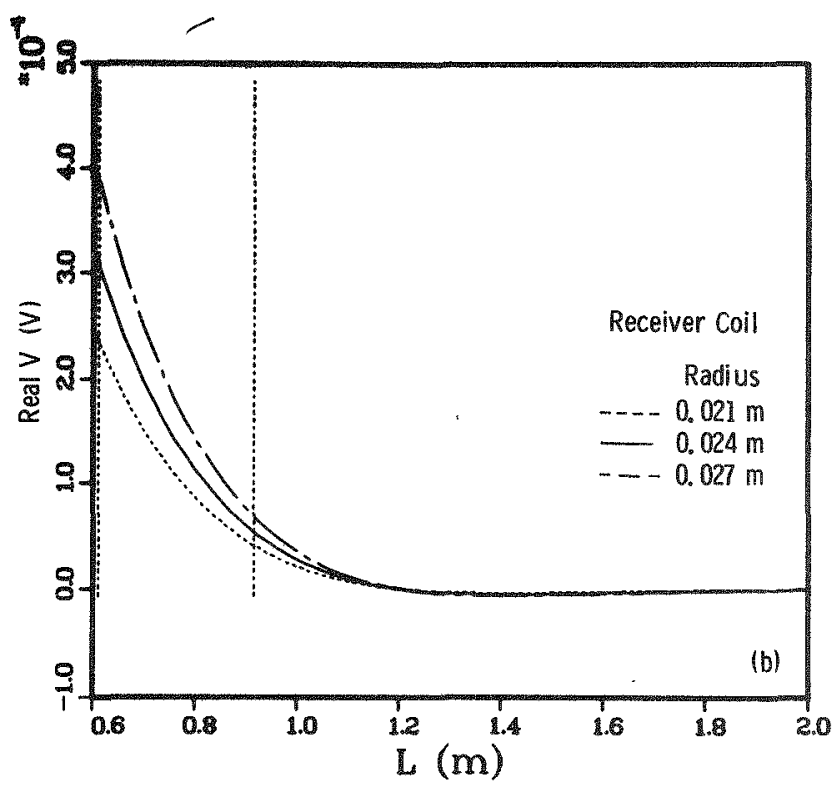

Figure 45. Real Part of the Complex Voltage in a Receiver Coil at Axial Distance L From the Dipole Source for Test Problem $E$ 

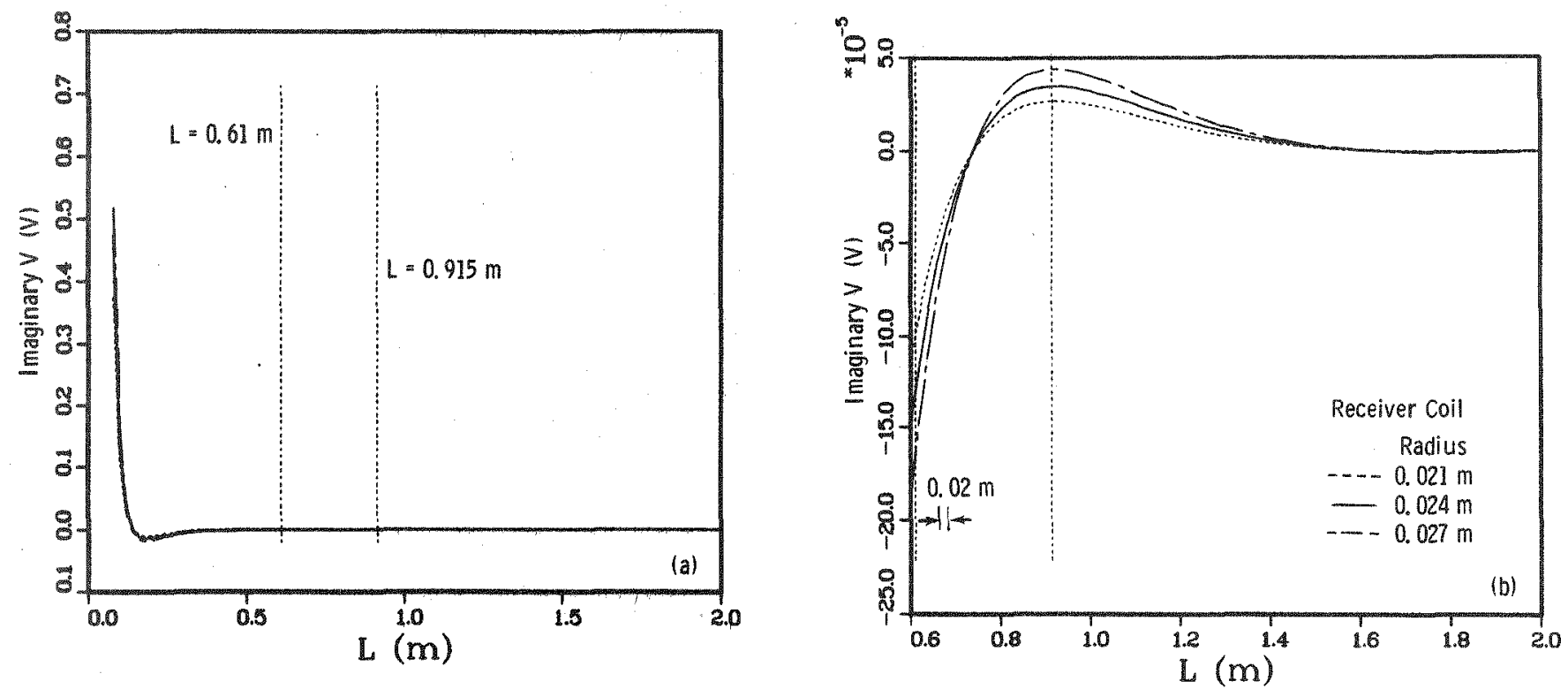

Figure 46. Imaginary Part of the Complex Voltage in a Receiver Coil at Axial Distance L From the Dipole Source for Test Problem E

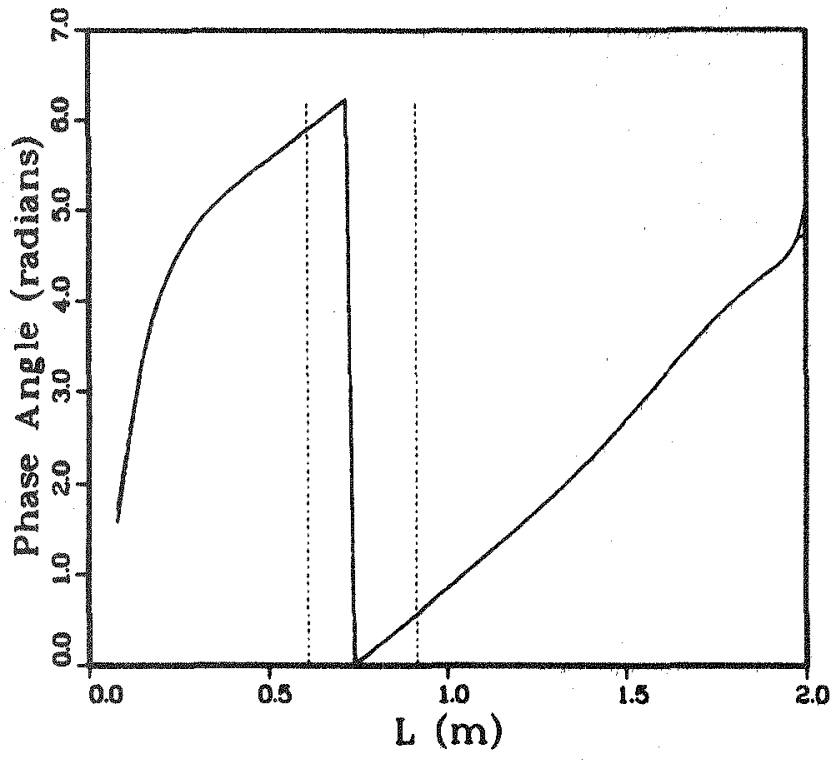

Figure 47. Phase Angle of the Complex Voltage in a Receiver Coil at Axial Distance L From the Dipole Source for Test Problem E (The three curves coincide for receiver coil radii $2.1,2.4$, and $2.7 \mathrm{~cm}$ )
Several alterations have been made to allow more detailed examination of the region near the source. Other alterations also occur to make some use of problems solved during computer code checkout. Modified problem parameters are listed below.

$$
\begin{array}{rlrl}
\text { DZSOUR } & =0.10 \mathrm{~m} & \text { Inside source region } \\
\text { DRSOUR } & =0.025 \mathrm{~m} & \epsilon=16.6 \epsilon_{\mathrm{o}} \\
\text { DT } & =0.002 & & \sigma=0.075 \mathrm{mho} / \mathrm{m} \\
\text { DZ } & =0.002 & \\
\text { NZ } & =201 & \\
N R & =201 & \\
\text { GAMMA } & =1.0 & \\
\text { RSET(1) } & =0.0256 & \\
\text { RSET(2) } & =0.11112 & \\
\text { RADIUS } & =0.0256 &
\end{array}
$$

Other parameters are as for Test Problem E. This treatment of the source region introduces horizontal layering. This example is labeled Modified Test E. Its graphical results are displayed in Figures 48-51. 


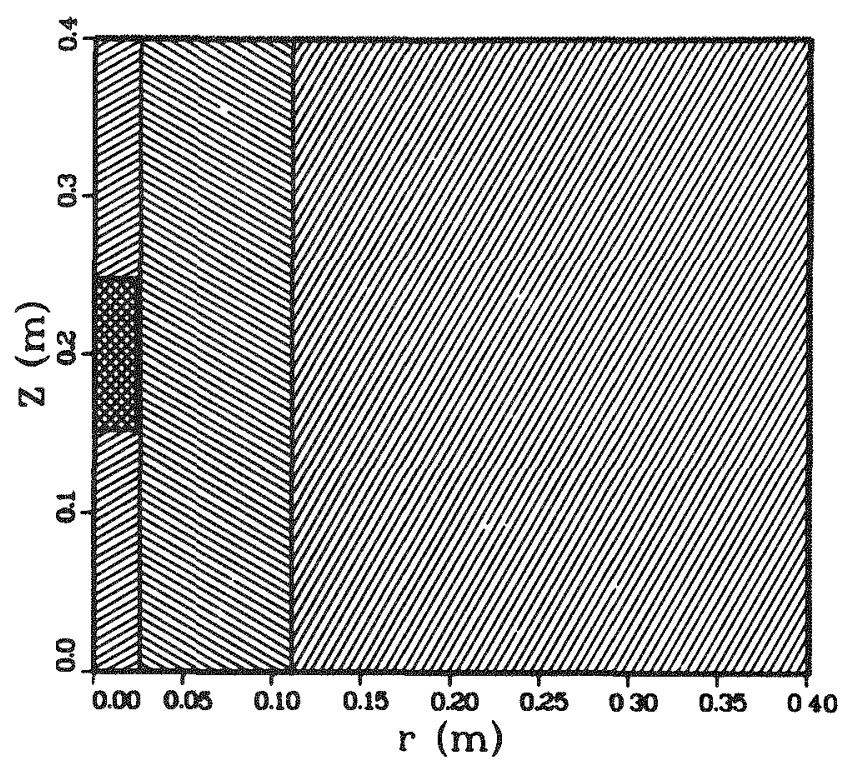

Figure 48. Soll-Layerng Geometry for Modıfied Test Problem E

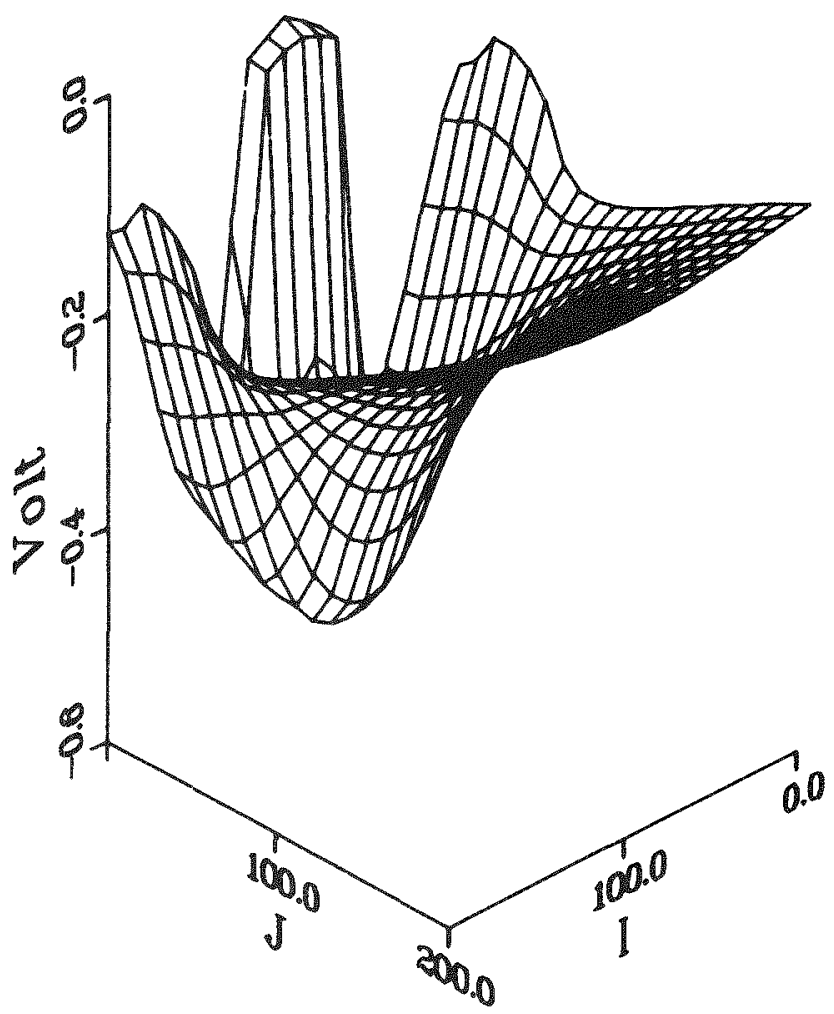

Figure 49. Real S( $/, \pi)$ Distribution for Modified Test Problem E
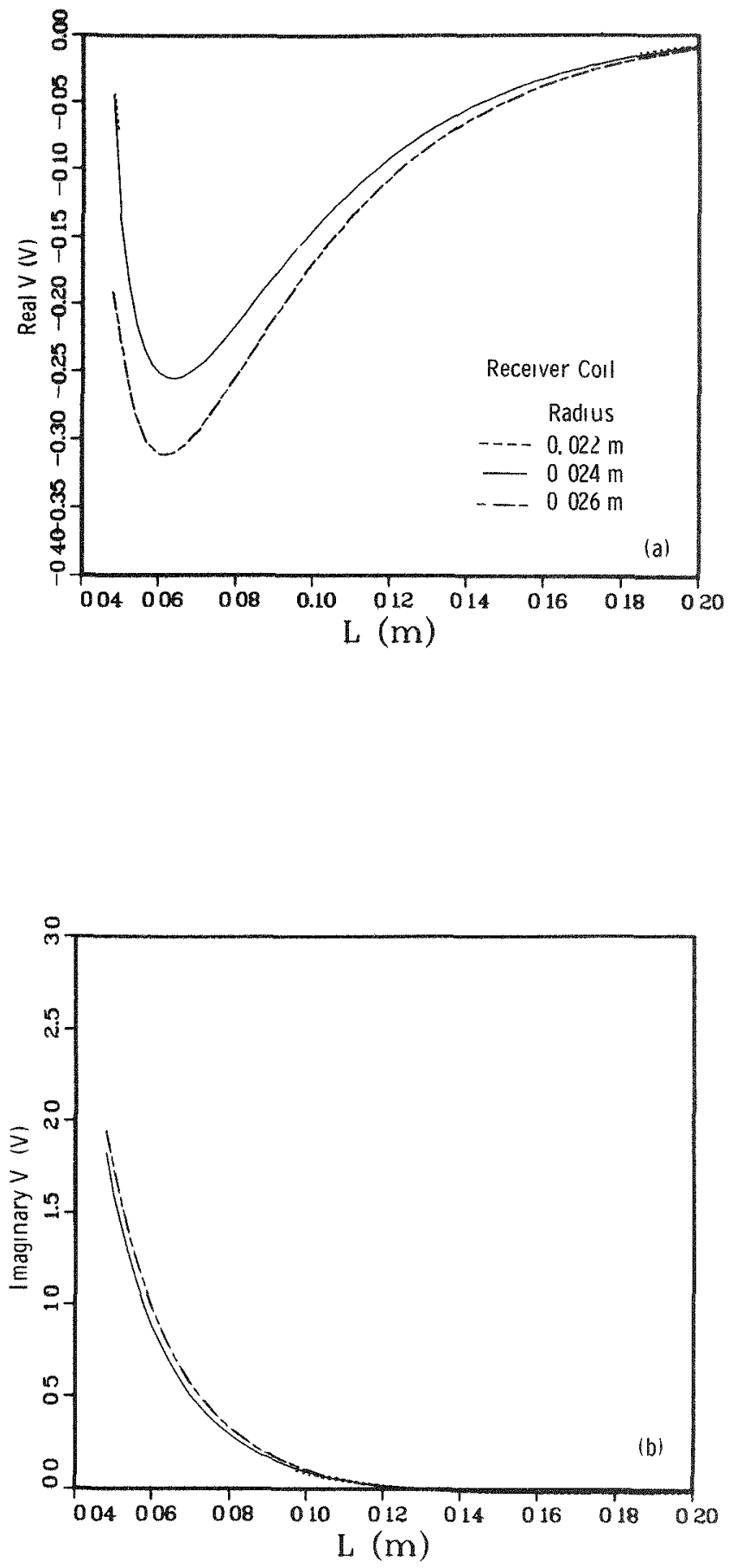

Figure 50. Real and Imaginary Parts of the Complex Voltage in a Receiver Coll at Axial Distance $L$ From the Dipole Source in Modified Test Problem E 


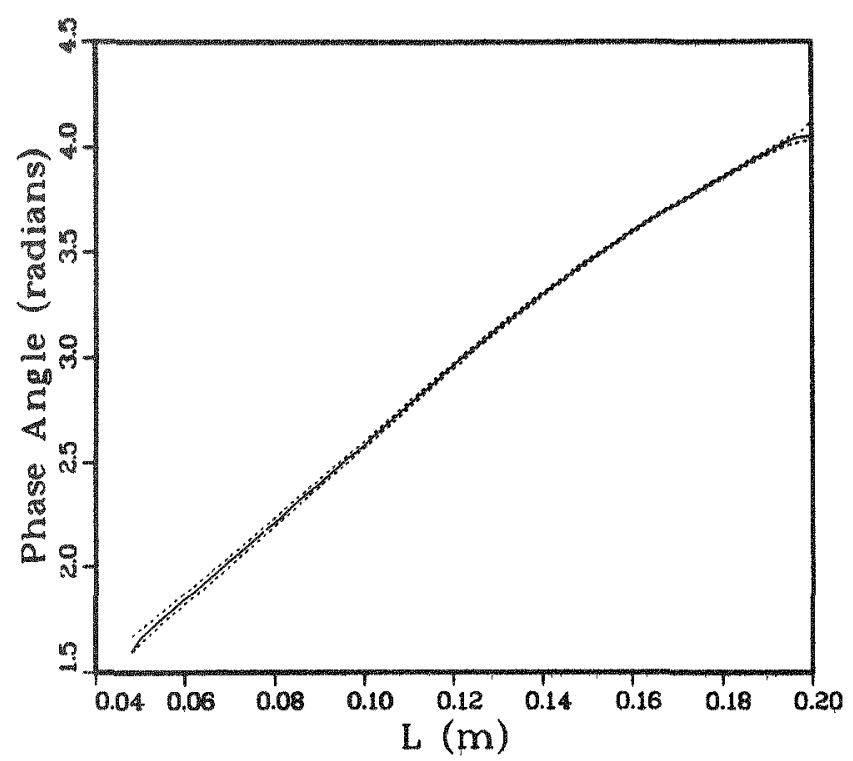

Figure 51. Phase Angle of the Complex Voltage in a Receiver Coil at Axial Distance L From the Dipole Source in Modified Test Problem $E$ (Results are similar for coil radii $2,1,2.4$, and $2.7 \mathrm{~cm}$. Boundary effects are seen near $L=0.20 \mathrm{~m}$ )

The basic difference with Test Problem $E$ is the differing electrical parameters inside the source region. Variation of $\operatorname{Real}(\mathrm{V})$ with iteration at $\mathrm{L}=0.18$ $\mathrm{m}$ is indicated in Figure 52. Although the test problem differs in several problem parameters (especially $\epsilon$ and $\sigma$ in the source region), its results are also shown in the figure.

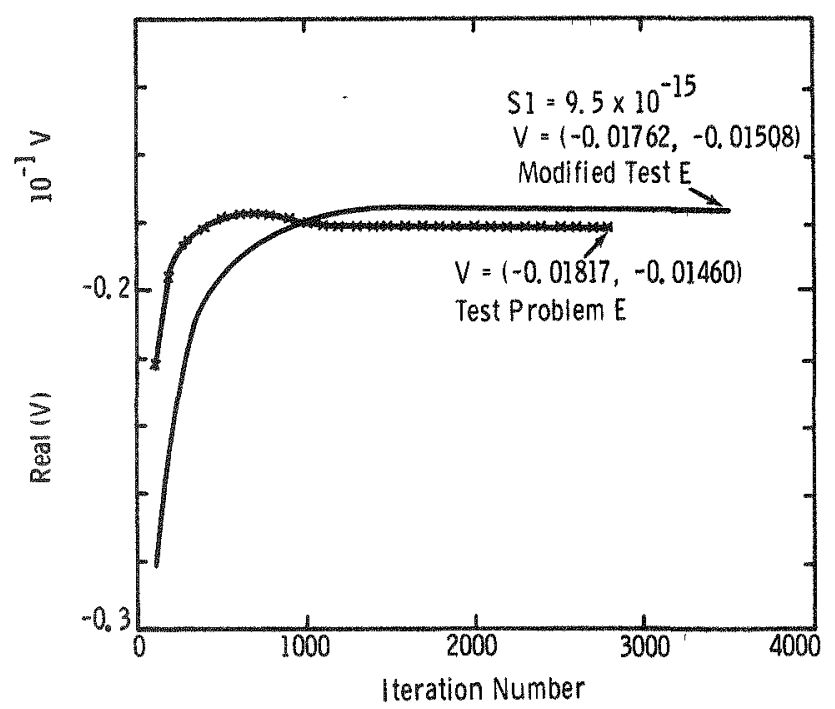

Figure 52. Real Part of the Complex Voltage in a Receiver Coil at $\mathrm{L}$ $=0.18 \mathrm{~m}$ for Test Problem $\mathrm{E}$ and Modified Test Problem E (Receiver radius is $2.4 \mathrm{~cm}$ )

\section{Test Problem F: Oil-Drilling Fluid, Frequency $30 \mathrm{MHz}$}

Earth electrical parameters

$\begin{array}{crl}\text { Region } & \epsilon / \epsilon_{\mathrm{O}} & \\ & 1.0 & \\ \text { I } & 1 . \mathrm{mho} / \mathrm{m}) \\ \text { II } & 2.0 & 1 . \mathrm{E}-10 \\ \text { III } & 10.0 & 0.0038\left(2.3 \omega \epsilon_{\mathrm{o}}\right)\end{array}$

These are altered by the code statements in Subroutine EP. Oil is now the drilling fluid (Region II).

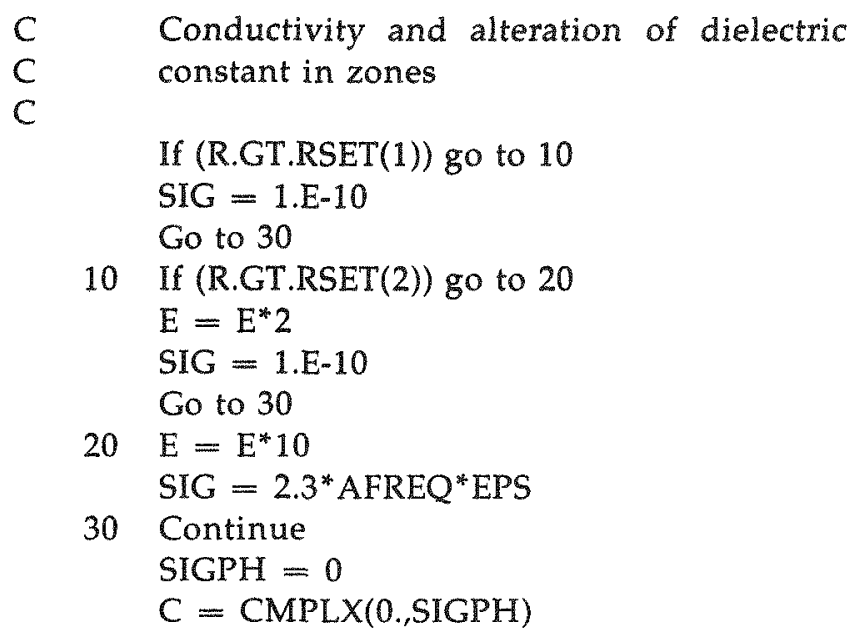

Additional code changes follow:

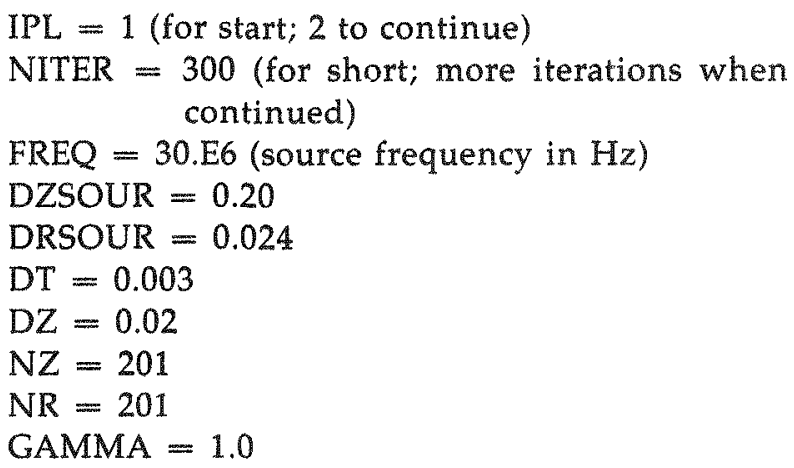

Other problem parameters are as for Test Problem C (and all the other test problems).

Figure 53 indicates variation of the convergence parameter with iteration number. At 3700 iterations the maximum percent difference between successive values of the real part of $S$ is $0.0026 \%$. This occurs at I $=2$ and $J=200$ in the mesh, suggesting that the remote corners of the spatial mesh have the most 
difficulty in converging. As further evidence of convergence, Figure 54 indicates that Real(V) becomes constant (within three significant figures) after 3000 iterations.

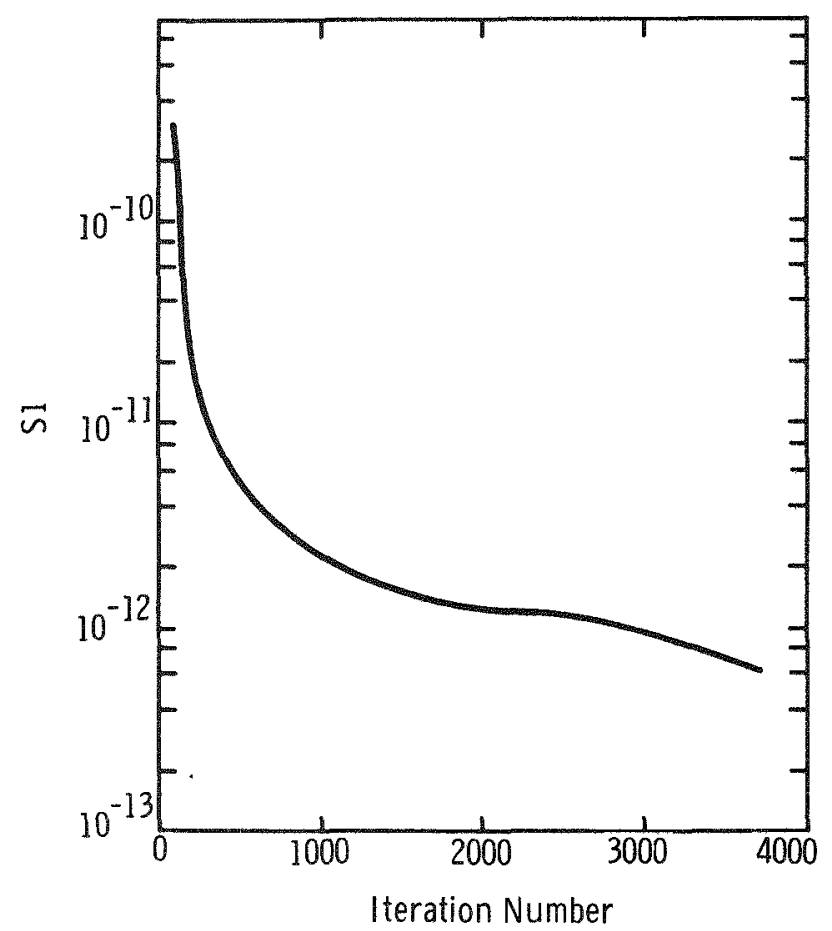

Figure 53. Variation of Convergence Parameter S1 With Iteration Number for Test Problem F

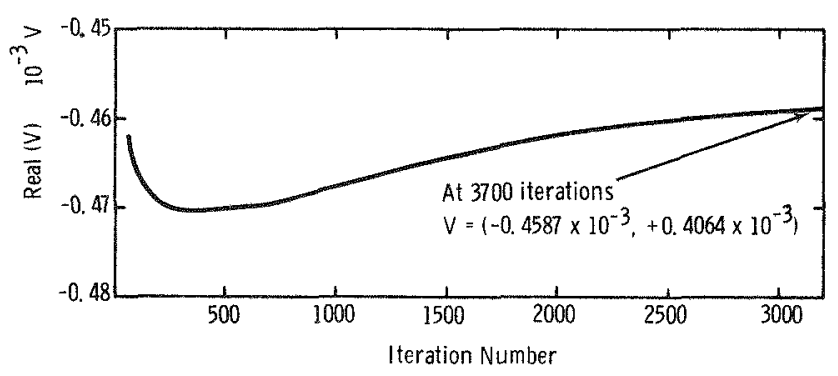

Figure 54. Real Part of the Complex Voltage in a Receiver Coil at $\mathrm{L}=0.92 \mathrm{~m}$ for Test Problem $\mathrm{F}$ (Receiver radius is $2.4 \mathrm{~cm}$ )

The soil-layering diagram for Test Problem $\mathrm{F}$ is jdentical to Figure 16. Other graphical output is given in Figures 55 through 58.

\section{Comparison With Analytical Results}

J. S. Y $\mathbf{u}^{6}$ has provided results for Test Problem $\mathbb{E}$ and $\mathrm{F}$ at distance $\mathrm{L}=0.61 \mathrm{~m}$ and receiver coil radius $0.0254 \mathrm{~m}$.

$$
\begin{array}{ll}
\text { Problem E } & \mathrm{V}=-3.5192 \mathrm{E}-4,-1.1065 \mathrm{E}-4 \\
\text { Problem F } & \mathrm{V}=+7.4628 \mathrm{E}-4,+1.7864 \mathrm{E}-3
\end{array}
$$

These real components are of opposite sign to those

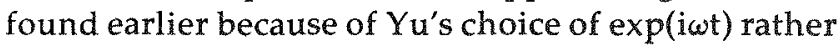
than $\exp (-i \omega t)$ time dependence. Figures $45-46$ give

Problem $\mathrm{E} \quad \mathrm{V}=+3.48 \mathrm{E}-4,-1.47 \mathrm{E}-4$

Interpolation directly from Figures 56-57 gives

$$
\text { Problem F } \quad \mathrm{V}=-7.40 \mathrm{E}-4,+1.77 \mathrm{E}-3 .
$$

The largest difference occurs in Imaginary (V) for Problem E. As seen in Figure 46, the variable is changing rapidly with $\mathrm{L}$, making $\mathrm{DZ}=0.02$ a little large for accurately following the variation. Since the Real(V) is changing less rapidly, it is expected to yield more reliable results.

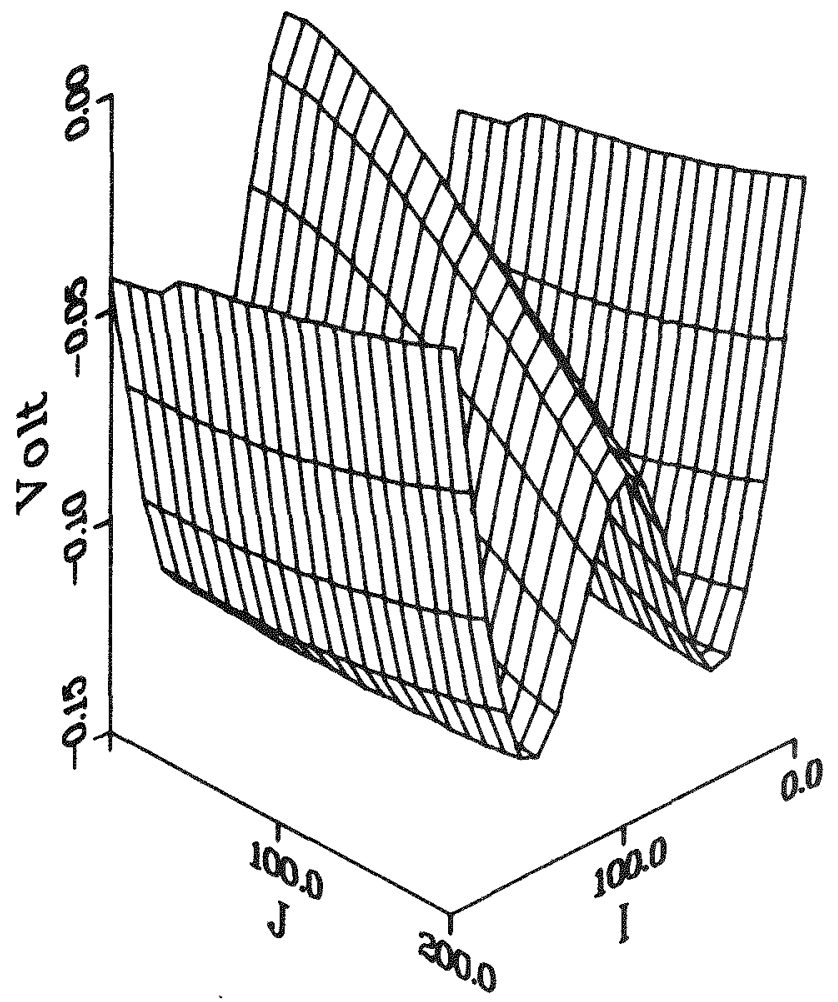

Figure 55. Real $S(z, r)$ Distribution for Test Problem F 
-
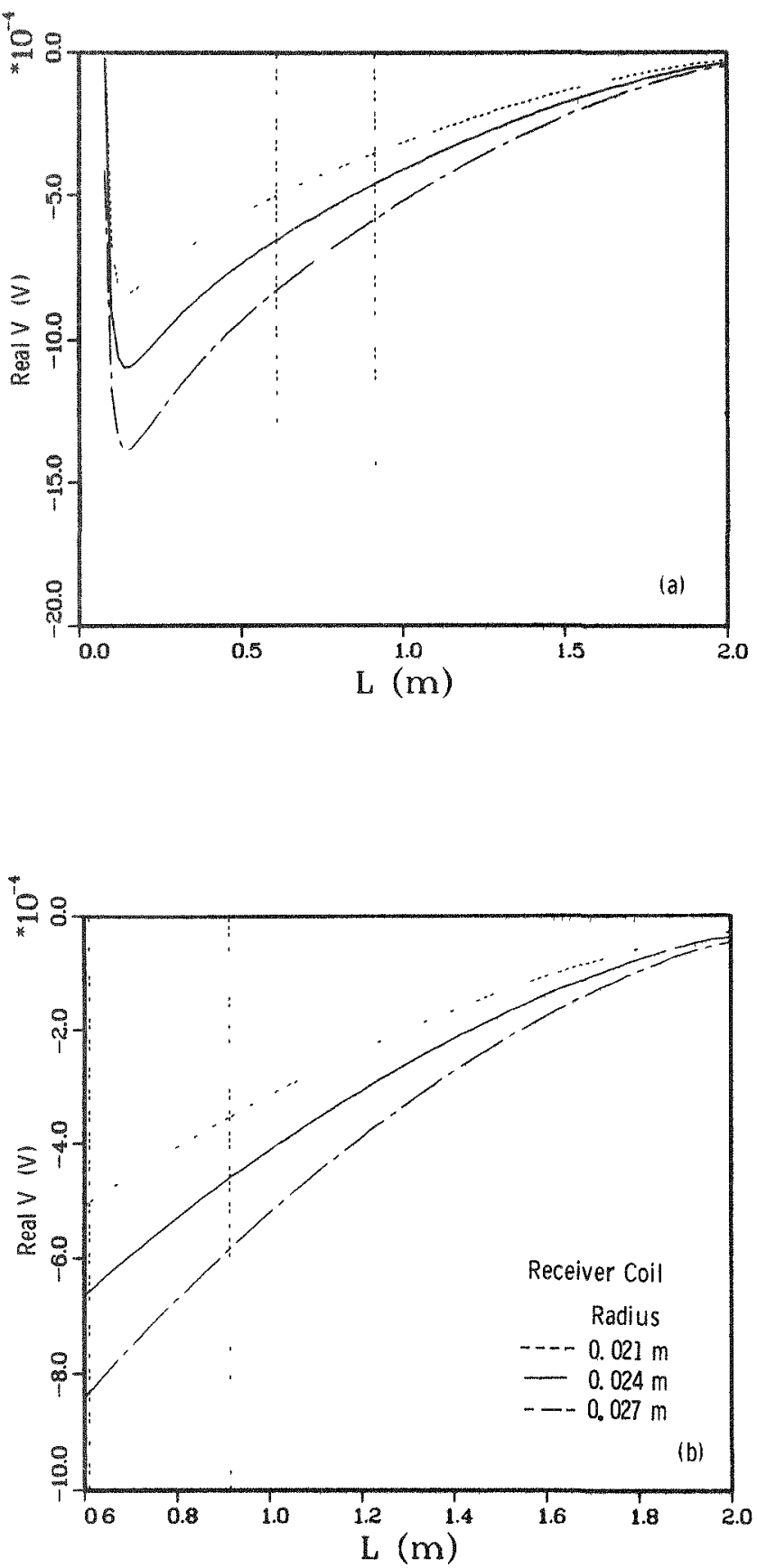

Figure 56. Real Part of the Complex Voltage in a Receiver Coil at Axial Distance L From the Dipole Source for Test Problem F
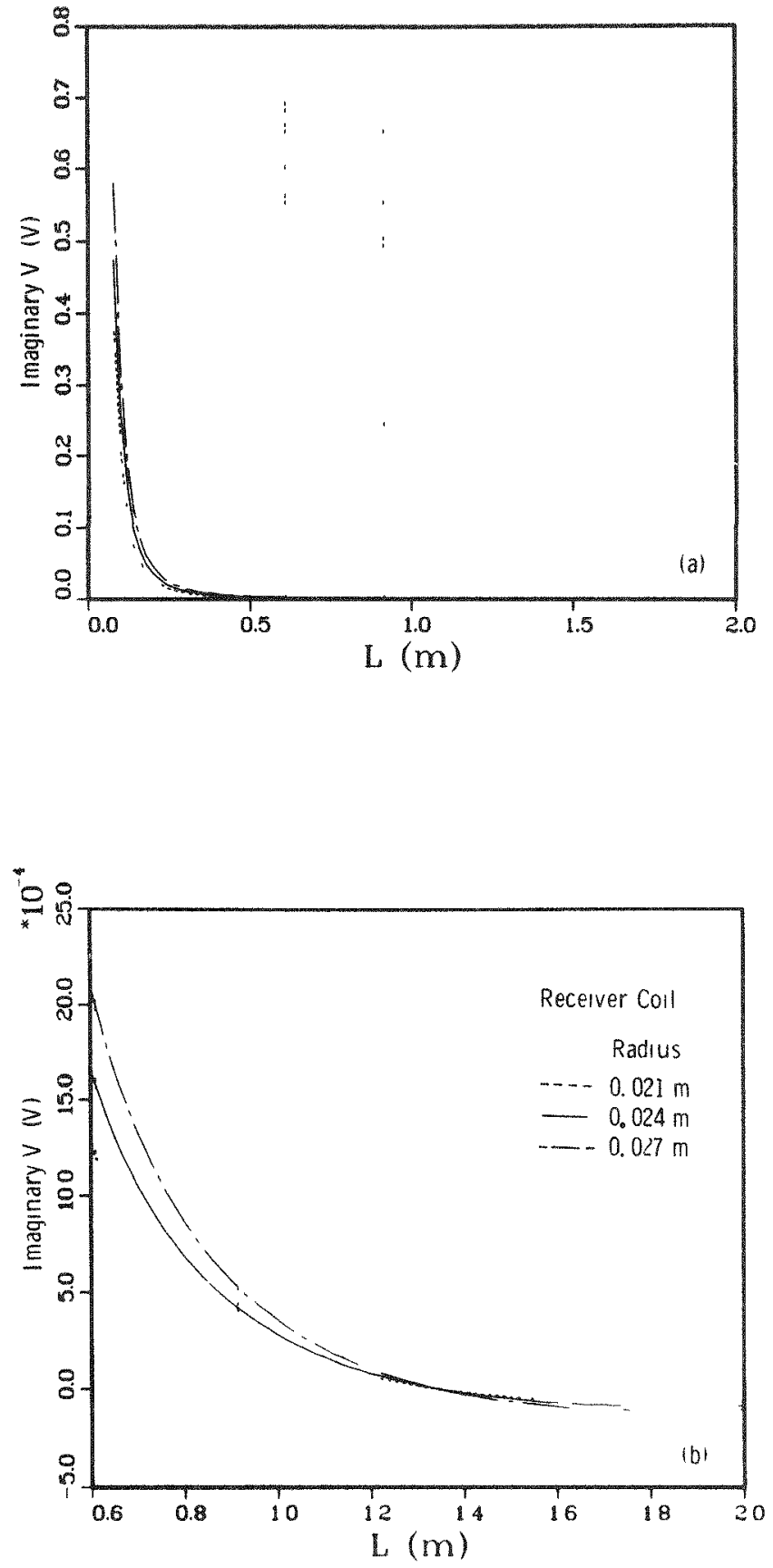

Figure 57. Imaginary Part of the Complex Voltage in a Receiver Coil at Axial Distance L From the Dipole Source for Test Problem F 


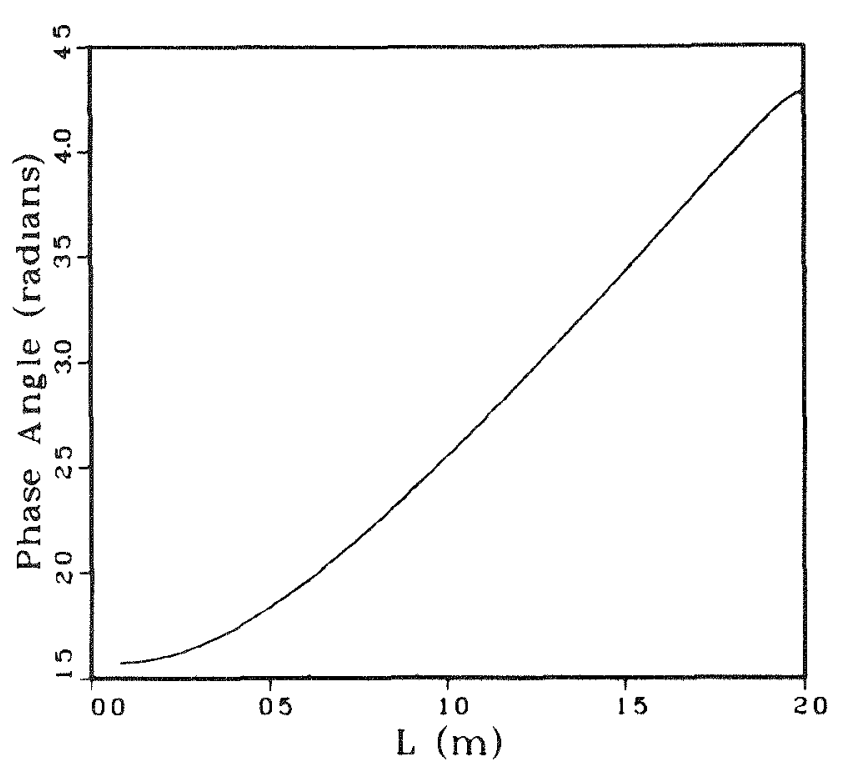

Figure 58. Phase Angle of the Complex Voltage in a Recelver Coul at Axial Distance L From the Dipole Source for Test Problem F (The three curves coincide for recelver coll radu 21,24 , and $27 \mathrm{~cm}$ )

Better agreement would be expected at $\mathrm{L}=0.915$ $m$ because of the less rapid variations there (see Figures 45-46 and 56-57). The variations in Figures 19-20 and 31-32 suggest that few percent agreement should be expected in these lower frequency test problems. Since the $\mathrm{L}=0.915 \mathrm{-m}$ separation is not being compared, the DZ could be reduced and Problem $E$ could be rerun for better resolution of Imagi$\operatorname{nary}(\mathrm{V})$.

\section{Possible Code Improvements}

The computer code used to solve the examples presented here can profit from a series of improvements. The most obvious ones are listed here. Perhaps you would like to add to the list or even implement some of the features. Because few problems have been processed so far, continued use of the code may reveal other, more pressing needs.

\section{Source Treatment}

Code generality can be increased by adding source options that maintain azimuthal symmetry, yet are not restricted to magnetic dipoles.

\section{Acceleration Scheme}

None of the test problems use the acceleration option described in Appendix A. It was tried on two test problems and produced instability in both cases.
Perhaps the iteration scheme can be altered to improve the rate of convergence.

\section{Mesh Size Alteration and Initial Guess Improvement}

When IPL is 3 (altering the mesh size from a previous run), a portion of the space where the old solution was found is used as the new source region. As an initial guess for a solution outside the new source region, the $S(I, J)$ are taken equal to that at the nearest point in the new source. If this option is used extensively, improvement in the initial guess may be justified. The guess can be improved by applying equations similar to those for evaluating boundary conditions. Such a change is expected to increase the rate of convergence in the larger spatial region.

The work of $\mathrm{Yu}$ and Reardon ${ }^{5}$ gives a solution for a vertically layered earth. Hence their solution could be used to provide the initial guess for a solution throughout the mesh. Iteration to the correct solution when horizontal layering is present should proceed much faster.

\section{Finite-Element Approach}

In the finite-element method of solution, the unknown function (S) is expanded as a summation of trial functions. Each set of trial functions is unique to each element of the domain where a solution is sought. Boundary elements can profit from choice of trial functions that have reasonable behavior at larger distances. Use of such an approach should improve code speed and may improve accuracy. A gain in robustness may also occur.

\section{Robustness}

A computer code is robust if it exhibits wide tolerance for input blunders and data inconsistencies. It implies little or no loss of computational accuracy over a wide range of parametric variation and lack of sensitivity to roundoff error. Robustness is gained by taking defensive action against all likely disasters (such as growth of S1 with iteration number), and providing an escape short of computer dump and job termination. The code's robustness can probably be increased in several areas. Continual growth of $S 1$ could be countered by problem restart with parameter adjustment designed to improve convergence. Convergence criteria could be chosen and parameters adjusted to allow continuation until convergence occurs, eliminating the need for problem restart. More disaster experience with the code will identify other areas. 


\section{Documentation}

A "law" of computing software is that nearly all programs are badly documented by their authors. Since the author and user have different views of the work, the best documentation has strong participatron by at least one user. Documentation should be provided at three levels: for software maintenance, for the code user, and for the analyst. Complete documentation and readily understood and used documentation are often conflicting requirements. The present documentation is directed toward the code user and the analyst. Extensive user experience should allow improvements in that portion of the documentation.

At times FIDELE may be used by personnel who are not programmers. An attempt has been made to allow a user to concentrate upon the problem at hand rather than upon the intricacies of computer commands. Appendices $E$ and $F$ try to direct the user's concentration upon inserting his particular problem. However, use of the code at present requires an elementary understanding of the NOS computing system, its XEDIT ablities at SNLA, and sufficient FORTRAN knowledge to adapt the EP subroutine so that the proper dielectric constant and conductivity are assigned to the two-dimensional layered earth.

\section{References}

1 I A Stratton, Electromagnetic Theory (New York McGraw-Hill Book Company, 1941), p 437

2. J W Sheldon, lterative Methods for the Solution of Elliptic Partial Differential Equations, Mathemattcal Methods for Digutal Computers, ed A Ralston and H S Wilf (New York John Wiley \& Sons, 1960)

3 I E Boers, SNEER A Digital Computer Program for the Simulation of Charged Particle Beams and Electrostatic Lenses, SAND79-0597 (Albuquerque Sandra National Laboratories, 1979)

4 J H Moran and K S Kunz, "Basic Theory of Induction Logging and Application to Study of Two-Coil Sondes," Geophystcs XXVIII(6), Part $1829-858$ (December 1962)

5 I S Yu and $\mathrm{P}$ Reardon, Effects of Borehole Fluds and Invaston Proftles Studied by a Fast-Convergent N-Zone Solution (to be published by Sandia National Laboratories, Albuquerque, NM)

6 Private communication, J S Yu, Div 4733, Sandia National Laboratories, December 3, 1980

7 E L Wachspress, Iteratroe Solution of Elliptic Systems and Applicathons to the Neutron Diffusion Equations of Reactor Physics (New Jersey Prentice Hall, Inc, 1966), p 111 
-

- 


\section{APPENDIX A}

\section{An Acceleration Scheme}

Wachspress ${ }^{7}$ suggests an acceleration scheme that may be used to increase the rate of convergence of SOR methods. These SOR methods apply most directly to consistently ordered systems of equations. A glance at our mesh is sufficient to establish consistency. Following Wachspress ( $p$ 107), we

1. Sketch the mesh and label the points in order of improvement (see Figure A-1 for example).

2. Note that in the five-point formula used, the order of improvement for any point uses values at neighboring points for a different but constant order of improvement.

Application of this ordering test to the first iteration scheme attempted indicates that the equations were not consistently ordered.

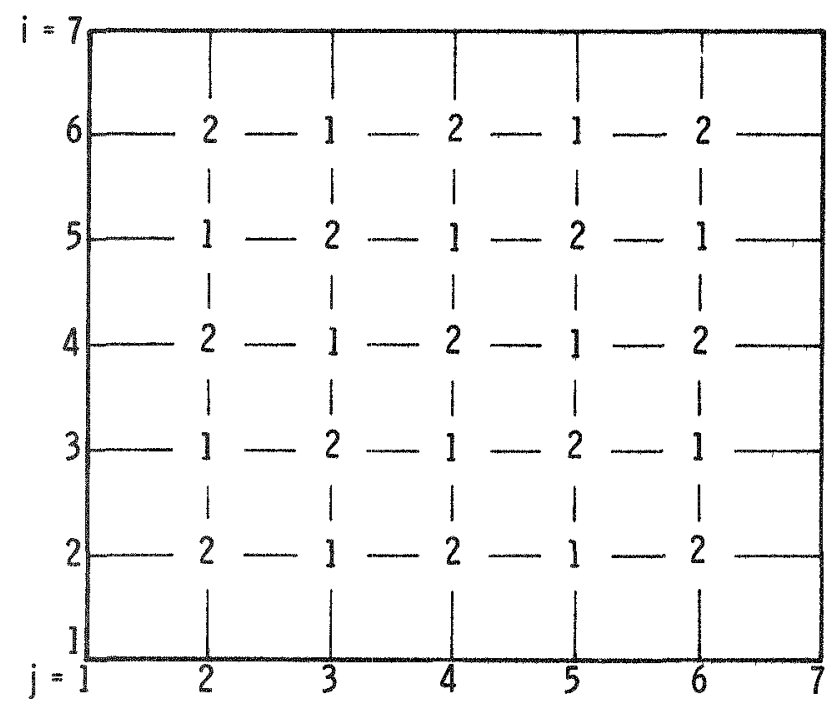

Figure A-1. Order of Improvement for Mesh Points in IMAX = $J \mathrm{MAX}=7$ Case

Following Wachspress ${ }^{7}$ (Section 4.6.2), we define the rate of convergence at iteration $t$ by

$$
\left[\mu^{(t)}\right]^{2}=\frac{\sum_{i, j}\left|S_{i, j}^{t}-S_{i, j}^{t-1}\right|^{2} \zeta_{i, j}}{\sum_{i, j}\left|S_{i, j}^{t}-S_{i, j}^{t-2}\right|^{2} \zeta_{i, j}} .
$$

We calculate the parameter

$$
\mu^{(\mathrm{t})}\left(\mathrm{M}_{\mathrm{B}}\right)=\frac{\mu^{(\mathrm{t})}+\mathrm{p}-1}{\mathrm{p}\left[\mu^{(\mathrm{t})}\right]^{1 / 2}}
$$

The new relaxation parameter is then given by

$$
\mathrm{p}^{\prime}=\frac{2}{1+\sqrt{1-\mu^{(\mathrm{t})}\left(\mathrm{M}_{\mathrm{B}}\right)^{2}}}
$$

The $\mathrm{p}^{\prime}$ is greater than $\mathrm{p}$. It is important to choose an initial value of $p$ that is less than the optimum value.

Direct application of this scheme to Test Problem A results in approximate convergence at 240 iter ations. Comparison with results in Table 2 gives $\mathrm{S}=$ $(-0.22 \mathrm{E}-4,+0.39 \mathrm{E}-3)$; i.e., much more rapid convergence.

\section{Sensitivity to Starting Value of Relaxation Parameter}

Results of $\mathrm{p}$-variation tests are presented in Table A-1. The trials were performed on Test Problem A. When the initial value of $p$ was 1.7 or 1.9 , the parameter was allowed to change only after 60 iterations. Then convergence was tested and $p$ was altered every 20 th iteration. The problem with initial $p=1.7$ required 280 iterations for convergence. With $p_{i}=$ 1.5 (1.9), convergence required 240 (220) iterations. From this table the initial value $p=1.9$ was set for subsequent computer runs. Additional iterations do not always reduce S1. When $p$ is altered by acceleration schemes, the speed of convergence does not appear to depend significantly upon the initial value of $\mathrm{p}$. 
Table A-1. Sensitivity of Convergence Parameter to the Initial Relaxation

Parameter

$\begin{array}{ccccccc}\begin{array}{c}\text { Iteration } \\ \text { Number }\end{array} & & & & & & \\ \mathbf{k} & \mathbf{p} & \mathbf{S 1} & \mathbf{p} & \mathbf{S 1} & \mathbf{p} & \mathbf{S 1} \\ 20 & 1.50 & 0.91 \mathrm{E}-10 & 1.70 & 0.13 \mathrm{E}-9 & 1.90 & 0.34 \mathrm{E}-7 \\ 40 & 1.78 & 0.86 \mathrm{E}-10 & 1.70 & 0.35 \mathrm{E}-10 & 1.90 & 0.18 \mathrm{E}-9 \\ 60 & 1.88 & 0.71 \mathrm{E}-10 & 1.70 & 0.17 \mathrm{E}-10 & 1.90 & 0.16 \mathrm{E}-10 \\ 80 & 1.92 & 0.42 \mathrm{E}-10 & 1.91 & 0.97 \mathrm{E}-11 & 1.94 & 0.68 \mathrm{E}-11 \\ 100 & 1.94 & 0.22 \mathrm{E}-10 & 1.94 & 0.54 \mathrm{E}-10 & 1.96 & 0.76 \mathrm{E}-11 \\ 120 & 1.95 & 0.13 \mathrm{E}-10 & 1.95 & 0.29 \mathrm{E}-10 & 1.97 & 0.70 \mathrm{E}-11 \\ 140 & 1.96 & 0.75 \mathrm{E}-11 & 1.96 & 0.14 \mathrm{E}-10 & 1.97 & 0.42 \mathrm{E}-11 \\ 160 & 1.97 & 0.35 \mathrm{E}-11 & 1.96 & 0.78 \mathrm{E}-11 & 1.97 & 0.16 \mathrm{E}-11 \\ 180 & 1.97 & 0.13 \mathrm{E}-11 & 1.97 & 0.39 \mathrm{E}-11 & 1.97 & 0.48 \mathrm{E}-12\end{array}$

\section{Sensitivity to Frequency of Adjusting $p$ and Testing for Convergence}

The process of adjusting the relaxation parameter with acceleration schemes requires additional program execution. In some applications the actual adjustments may be small and may not significantly speed convergence. Thus the possibility exists that "after every $n_{\mathrm{sr}}$ th iteration" is the optimum interval for altering the value of the relaxation parameter $p$. Table A-2 summarizes the results of numerical study of the effect.
In the $n_{\mathrm{sr}}=20$ and 30 cases, changes in $\mathrm{p}$ were allowed only after an initial 60 iterations. If too few iterations are allowed, the S1 values are not sufficiently stable. When $n_{\text {sr }}=10$ or 15 , this resulted in computer-code attempts to extract the square root of a negative number in evaluating $\mathrm{Eq}(\mathrm{A}-3)$. The 60iteration limitation and increase of $n_{s \mathrm{r}}$ to 20 removed the problem. The computer time is that required for the entire problem, including convergence and $\sim 1$-s program compilation.

Table A-2. Sensitivity of Convergence to Frequency $\left(\mathbf{n}_{\text {sr }}\right)$ of Adjusting $\mathrm{p}^{*}$

$\begin{array}{cccc} & \mathbf{n}_{\text {Sr }}=20 & \mathbf{n}_{\text {Sr }}=30 & \mathbf{n}_{\mathbf{S r}}=50 \\ & \mathbf{S} 1 \mathbf{( k )} & \mathbf{S 1}(\mathbf{k}) & \mathbf{S 1 ~ ( k )} \\ & 0.76 \mathrm{E}-11(100) & 0.49 \mathrm{E}-11(90) & 0.43 \mathrm{E}-11(100) \\ & 0.13 \mathrm{E}-12(200) & 0.14 \mathrm{E}-12(210) & 0.32 \mathrm{E}-12(200) \\ \text { Computer Time (s) } & 129.0 & 136.7 & 141.7\end{array}$

${ }^{*} p_{1}=1.9$ 


\section{APPENDIX B}

\section{Sensitivity to Actual Value of $f$ at Outer Boundaries}

When problems are posed for an inhomogeneous earth, the value of $f$ is not available at the outer boundaries. However, the f values are expected to be small. Its errors are expected to influence $S$ only through second-order effects. As a test of this sensitivity, numerical studies of Test Problem A yielded the converged values of $S$ in Table B-1. The table indicates that $S$ values near the JMAX boundary may not be reliable. However, there is minimal influence of uncertainty in $f$ upon values of $S$ near the axis where receivers are located.

Table B-1. Sensitivity of $S$ to $f$ Variation

$\begin{array}{lccc} & S(I=1, J=1) & S(I=50, J=30) & S(I=50, J=99) \\ f_{o} & -0.14 \mathrm{E}-4,+0.80 \mathrm{E}-3 & -0.82 \mathrm{E}-5,+0.14 \mathrm{E}-2 & -0.22 \mathrm{E}-4,+0.39 \mathrm{E}-3 \\ \mathrm{f}^{\prime}=\mathrm{f}_{\mathrm{o}}{ }^{*} 1.1 & -0.14 \mathrm{E}-4,+0.80 \mathrm{E}-3 & -0.82 \mathrm{E}-5,+0.14 \mathrm{E}-2 & -0.22 \mathrm{E}-4,+0.38 \mathrm{E}-3 \\ \mathrm{f}^{\prime \prime}=\mathrm{f}_{\mathrm{o}}{ }^{*} 2 . & -0.14 \mathrm{E}-4,+0.80 \mathrm{E}-3 & -0.79 \mathrm{E}-5,+0.13 \mathrm{E}-2 & -0.21 \mathrm{E}-4,+0.29 \mathrm{E}-3 \\ \mathrm{f}_{\mathrm{o}} \text { implies } \mathrm{f} \text { is evaluated by } \mathrm{Eq}(24) \text { and }(27)\end{array}$

$\mathrm{p}_{1}=1.9, \mathrm{n}_{\mathrm{sr}}=20$, IMAX $=$ JMAX $=99$ 
-

- 


\section{APPENDIX C}

\section{Test Case for Nonuniform Radial Mesh}

This appendix furnishes results of a test for use of gamma other than 1. We recalculate Test Problem F by using a finer, nonlinear grid. The calculation confirms consistency with the earlier result and also reveals considerable lack of robustness with respect to input value of gamma. Alterations from Test Problem $F$ are indicated below:

$$
\begin{aligned}
\text { GAMMA } & =1.4 \\
\text { DZ } & =0.007 \\
\text { DT } & =0.004 \\
\text { NITER } & =200 \text { (for start, more iterations later) } \\
\text { IPL } & =1 \text { (for start, } 2 \text { to continue) } \\
\text { DZSOUR } & =0.10 \\
\text { DRSOUR } & =0.012
\end{aligned}
$$

Other parameters are as for Problem F. In steps of 20 iterations, the convergence parameter $\mathrm{S} 1 \mathrm{grew}$ as 180 , $1.19 \mathrm{E} 13,0.08 \mathrm{E} 23,5.74 \mathrm{E} 34,4.34 \mathrm{E} 45$. Examination of the initial guess (appropriate for a uniform medium) indicated $25 \%$ changes in $S$ at neighboring points on the $\mathrm{z}$ boundaries of the source region. Although not printed during this run (since ISHORT $=1$ ), the change in $S$ at the $r$ boundary is also expected to be large.

A new start used

$$
\begin{aligned}
\text { DZSOUR } & =0.10 \\
\text { DRSOUR } & =0.02 \\
\text { DZ } & =0.002 \\
\text { DT } & =0.003
\end{aligned}
$$

Here the $\mathrm{S} 1$ parameter grows as $0.0087,3.09 \mathrm{E} 8$, $1.90 \mathrm{E} 19,6.09 \mathrm{E} 29 \mathrm{in} 20$ iteration intervals. The convergence parameter is again misnamed. After 200 iterations, S1 had "settled down" to 2.9E93.

A change of GAMMA to 1.2 offered a little help with $S 1$ values growing as $0.0001,1.6,1.7 \mathrm{E} 4,1.8 \mathrm{E} 8$ in 20 iteration intervals. Changing to GAMMA $=1.1$ and DT to 0.006 suggested convergence, with $\mathrm{S} 1=$ $5.8 \mathrm{E}-8,1.0 \mathrm{E}-8,5.4 \mathrm{E}-9$, and $3.2 \mathrm{E}-10$ in 20 iteration intervals. Both of these changes (in given order) place the $r$ boundary farther from the source, suggesting some difficulty with the boundary condition at IMAX. There is also a possibility that a small value of DT (or DZ) may cause computer roundoff error large enough to make the solution diverge in some portions of the mesh.

The problem was restarted with parameters

$$
\begin{aligned}
\text { GAMMA } & =1.1 \\
\text { DZ } & =0.002 \\
\text { DT } & =0.006 \\
\text { NITER } & =1200 \\
\text { IPL } & =1 \\
\text { DZSOUR } & =0.10 \\
\text { DRSOUR } & =0.02
\end{aligned}
$$

Convergence is indicated in Figures $\mathrm{C}-1$ and $\mathrm{C}-2$. Comparison with the voltage in Figure 56 at coil radius $0.021 \mathrm{~m}$ indicates that the results are consistent with those found in Problem F. Other graphical results from the test of a nonuniform radial mesh are given in Figures C-3 through C-6.

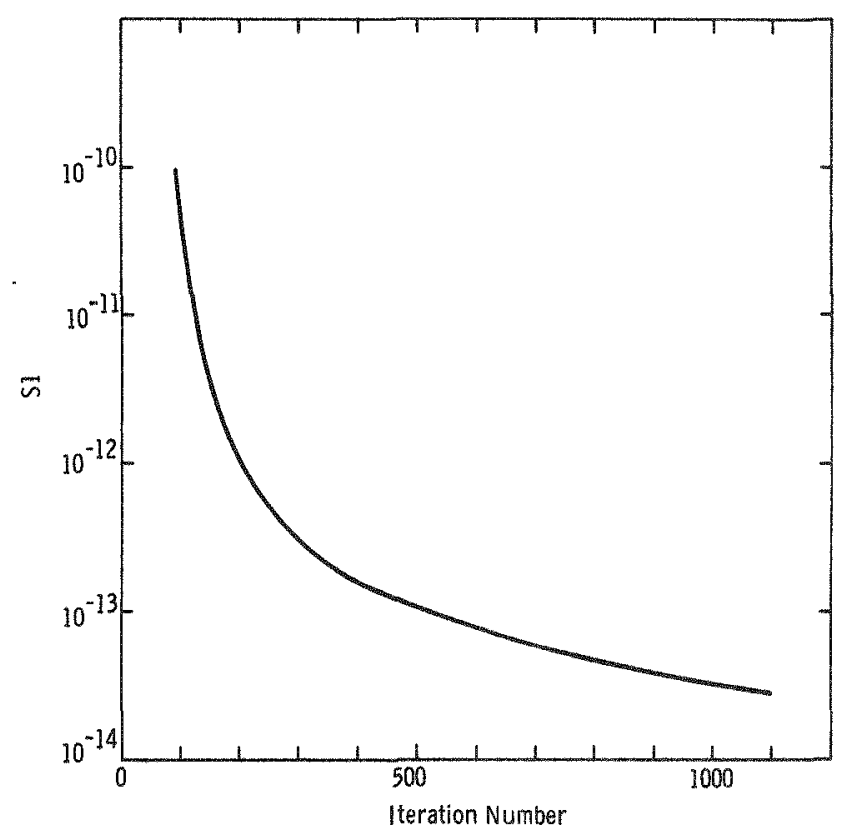

Figure C-1. Variation of Convergence Parameter S1 With Iteration Number for Test Problem G 


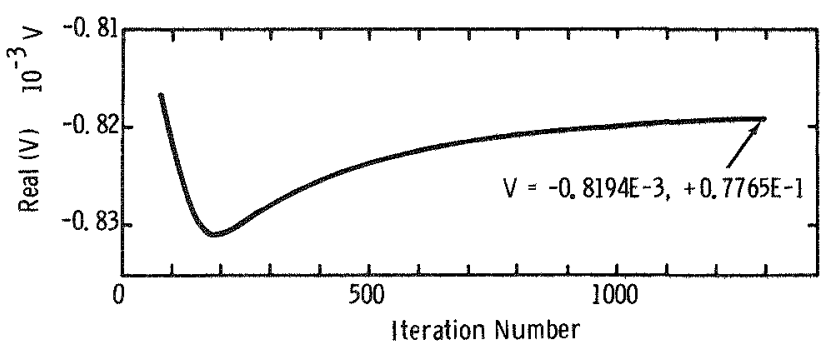

Figure C-2. Real Part of Complex Voltage in a Receiver Coil at $\mathrm{L}=$ $0.14 \mathrm{~m}$ for Test Problem G. (Coil radius is $0.0211 \mathrm{~m}$. At 2500 iterations, $\mathrm{V}$ becomes $-0.8181 \mathrm{E}-3,+0.7765 \mathrm{E}-1$.)

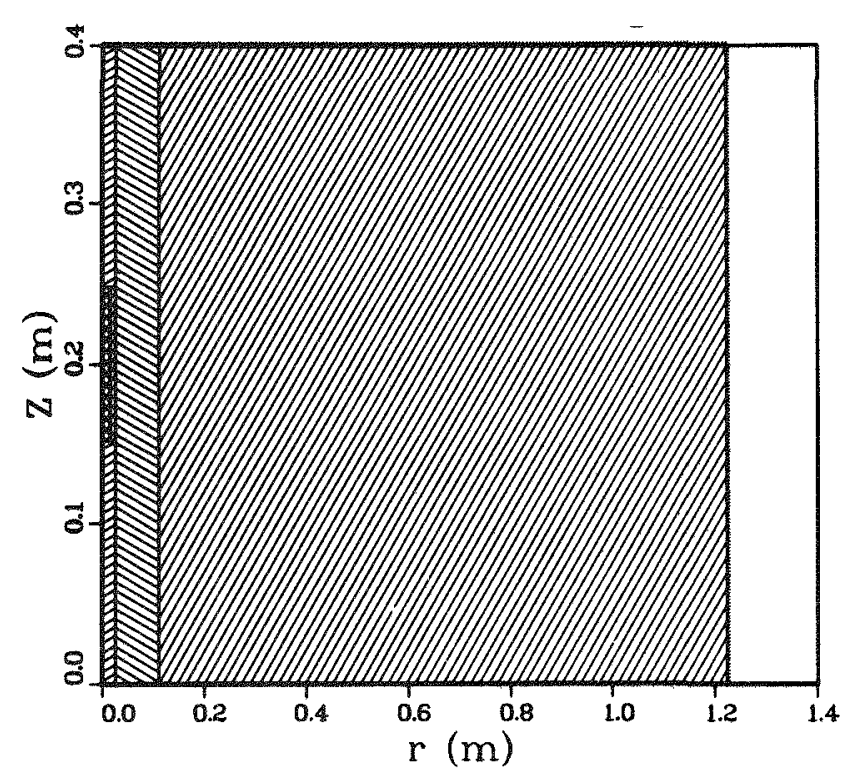

Figure C-3. Soil-Layering Geometry for Test of Nonuniform Radial Mesh

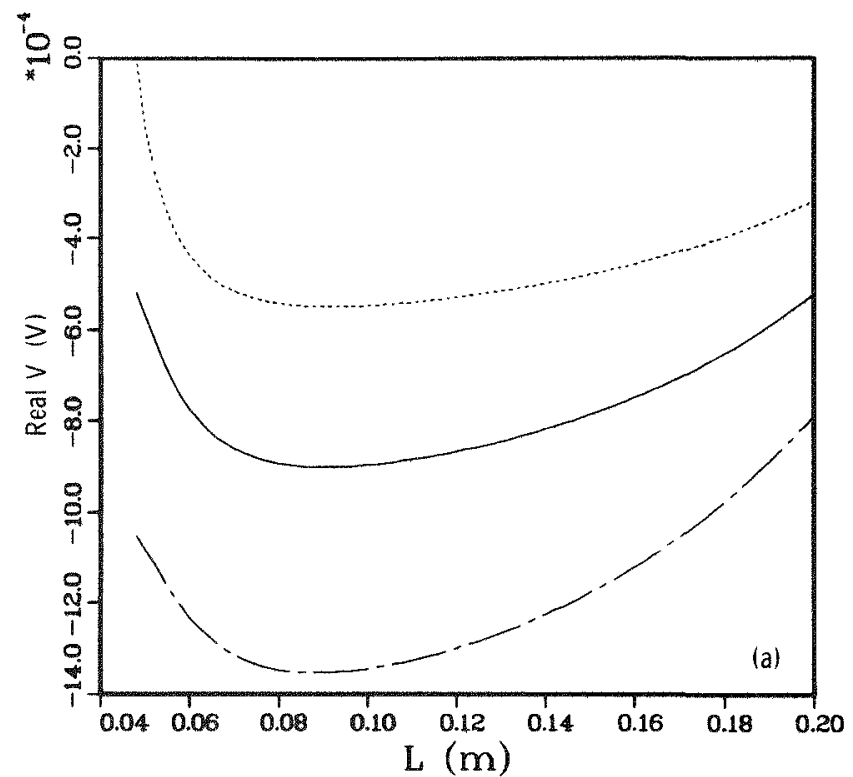

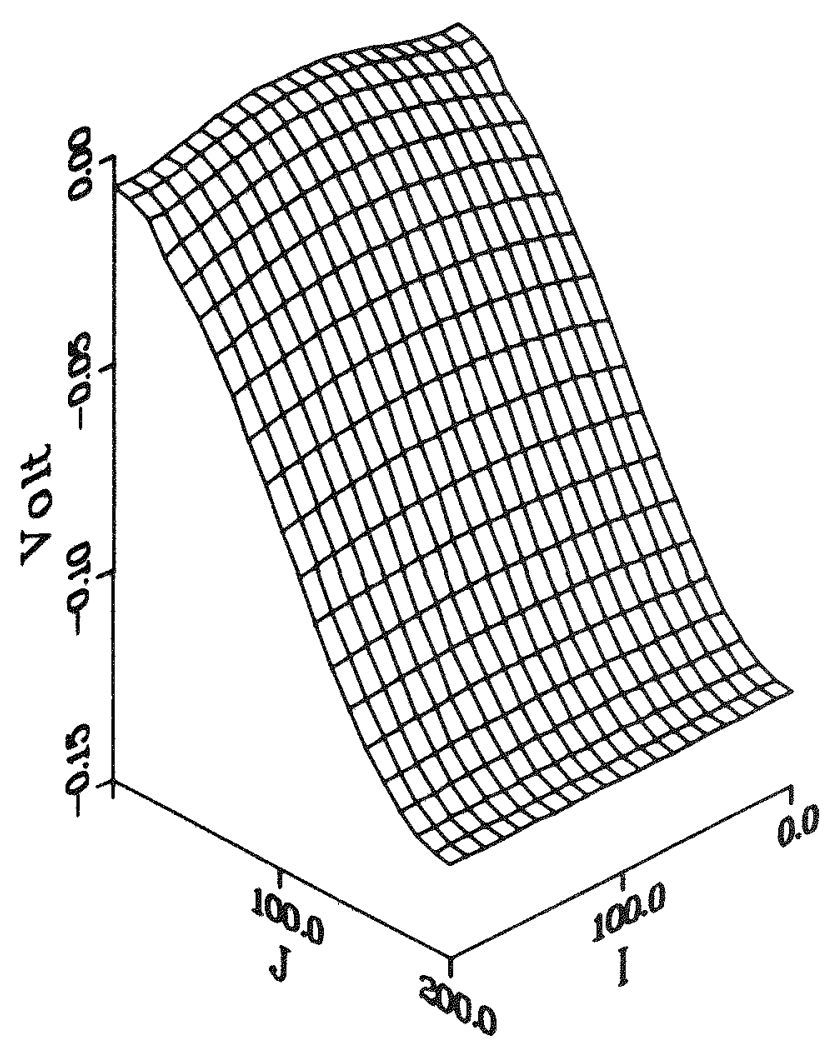

Figure C-4. Real $S(z, r)$ Distribution for Test of Nonuniform Radial Mesh

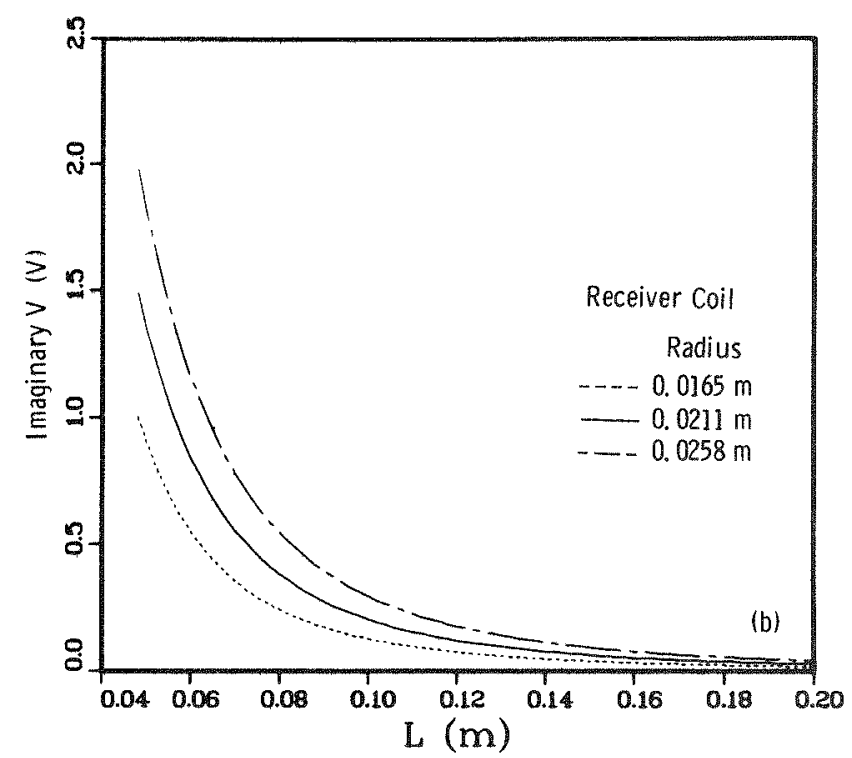

Figure C-5. The Complex Voltage in a Receiver Coil at Axial Distance L From the Dipole Source for Testing Nonuniform Radial Mesh 


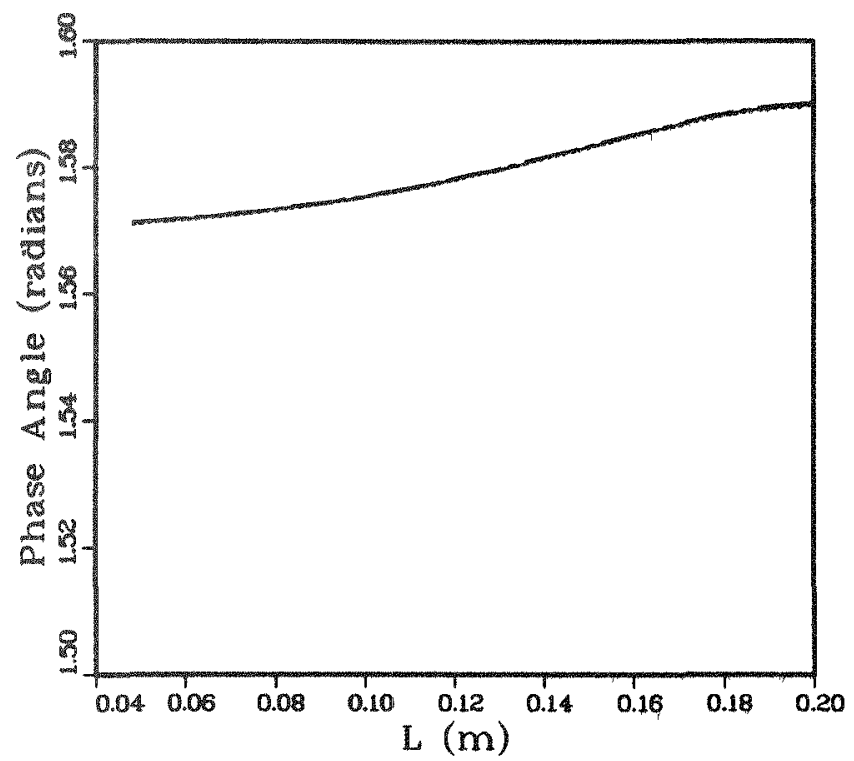

Figure C-6. Phase Angle of the Complex Voltage in a Receiver Coil at Axial. Distance L From the Dipole Source for Testing Nonuniform Radial Mesh. (Curves for receiver coil radii 0.0165, 0.0211, and $0.0258 \mathrm{~m}$ are not distinguishable.) 
-

- 


\section{APPENDIX D}

\section{Test Case for Mesh-Size Alteration After Convergence}

In this appendix our intent is to illustrate use of the IPL $=3$ parameter value that alters the mesh size, expanding the domain where the solution is found. We solve Test Problem F in a small region of space, then expand the space to find that results in the larger domain are consistent with the earlier results. However, note that robustness is lacking in this expansion. Some combinations of parameters that produce convergence in the initial domain may cause divergence in the expanded domain. When the result from Appendix $C$ is restarted with IPL $=3$, the convergence parameter S1 grows with iteration number, indicating a diverging "solution."

A chronological order is followed as an aid to code users. Input parameters chosen are:

$$
\begin{aligned}
\text { IPL } & =1 \\
\text { NITER } & =100 \\
\text { DZ } & =0.004 \\
\text { DT } & =0.002 \\
\text { GAMMA } & =1.0 \\
\text { DZSOUR } & =0.20 \\
\text { DRSOUR } & =0.024
\end{aligned}
$$

Other parameters are as for Test Problem F. Problem continuations alter IPL to 2 and NITER to 1200. Variation of $S 1$ and $\operatorname{Real}(\mathrm{V})$ at one location in the mesh are given in Figures D-1 and D-2. Convergence is clear after 2500 iterations.

After convergence is confirmed and the file of $S(I, I)$ values is preserved, the problem is continued with IPL $=3$, NITER $=100$ in a new computer run. As a precaution, it may be wise to save the next file under a new permanent-file name. This allows additional restarts (with IPL $=3$ ) from the converged solution in the smaller domain. Following continuations (with IPL returned to 2 ) should lead to convergence in the larger domain.

Continued iterations after the mesh change yields Figures D-3 and D-4. Convergence is apparent after about 4500 iterations. At $\mathrm{L}=0.912 \mathrm{~m}$ and 4900 iterations $\mathrm{V}=-0.4678 \mathrm{E}-3,0.4268 \mathrm{E}-3$. Linear interpolation between $\mathrm{L}=0.912$ and $\mathrm{L}=0.924 \mathrm{~m}$ in Figure $B-4$ gives $V=-0.4631 \mathrm{E}-3,0.4120 \mathrm{E}-3$ at $\mathrm{L}=0.92 \mathrm{~m}$. Comparison with Figure 54 (for Test Problem F) indicates percent differences of $0.48 \%$ and $1.40 \%$ in the real and imaginary parts of $\mathrm{V}$ at $\mathrm{L}=0.92 \mathrm{~m}$.

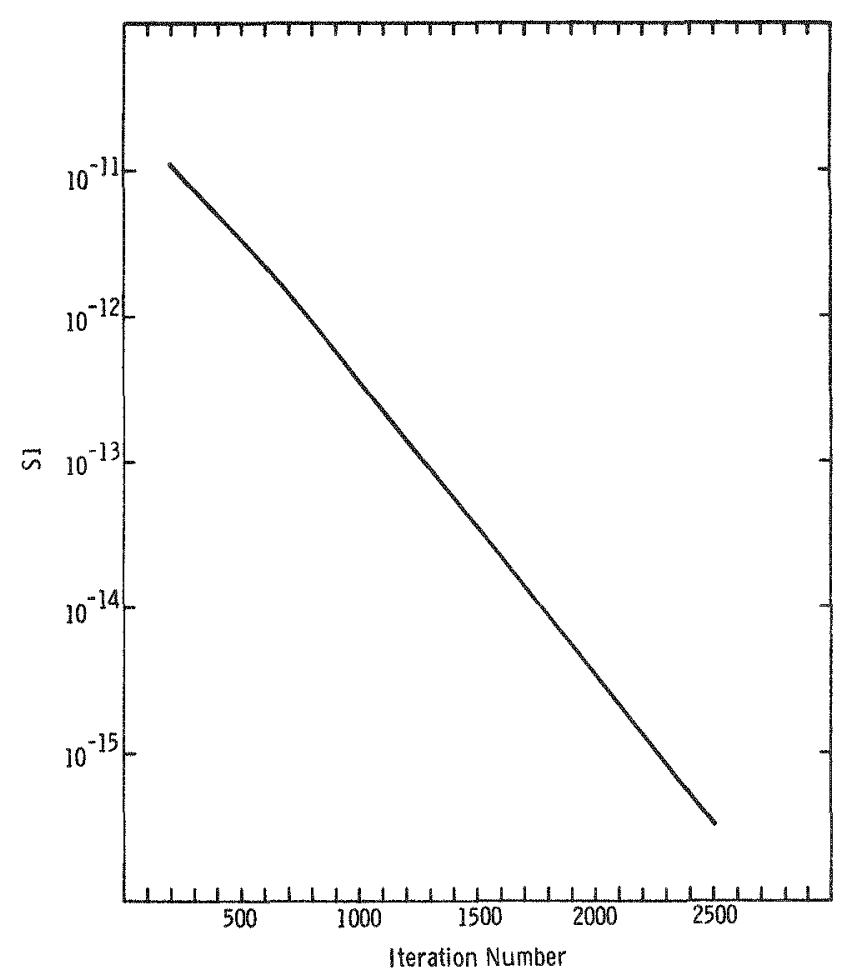

Figure D-1. Variation of Convergence Parameter S1 With Iteration Number for Test Problem $G^{\prime}$

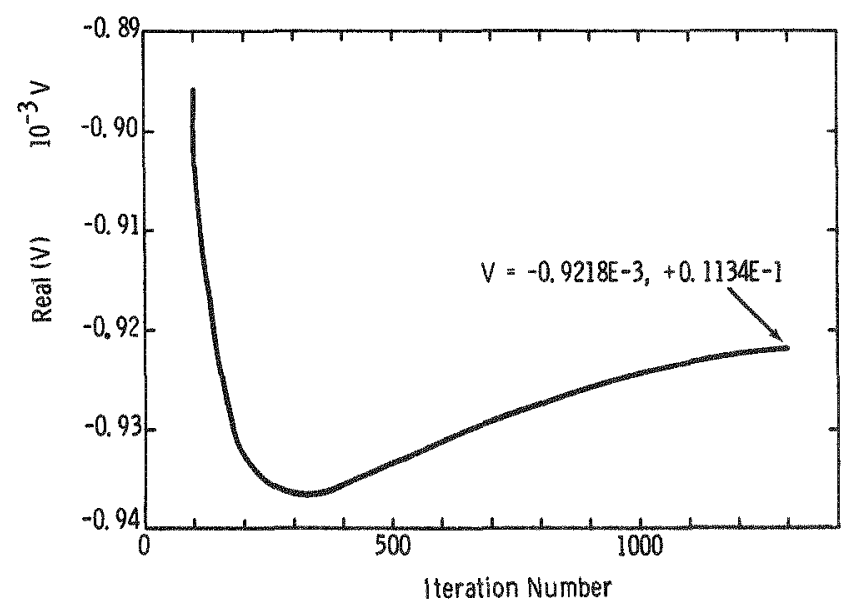

Figure D-2. Real Part of Complex Voltage in a Receiver Coil at $L=$ $0.30 \mathrm{~m}$ for Test Problem $\mathrm{G}^{\prime}$. (Coil radius is $0.024 \mathrm{~m}$. After 2500 iterations, $V=-0.9184 \mathrm{E}-3,+0.1134 \mathrm{E}-1$.) 


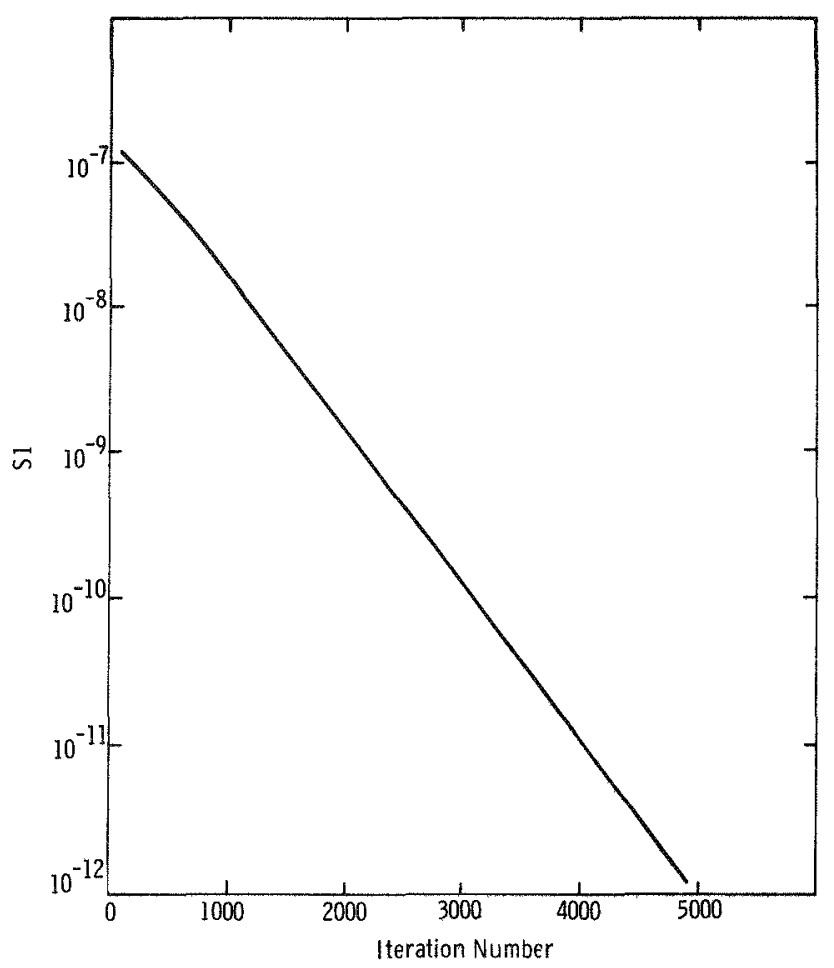

Figure D-3. Variation of Convergence Parameter S1 With Iteration Number for Test Problem G' After Mesh Expansion

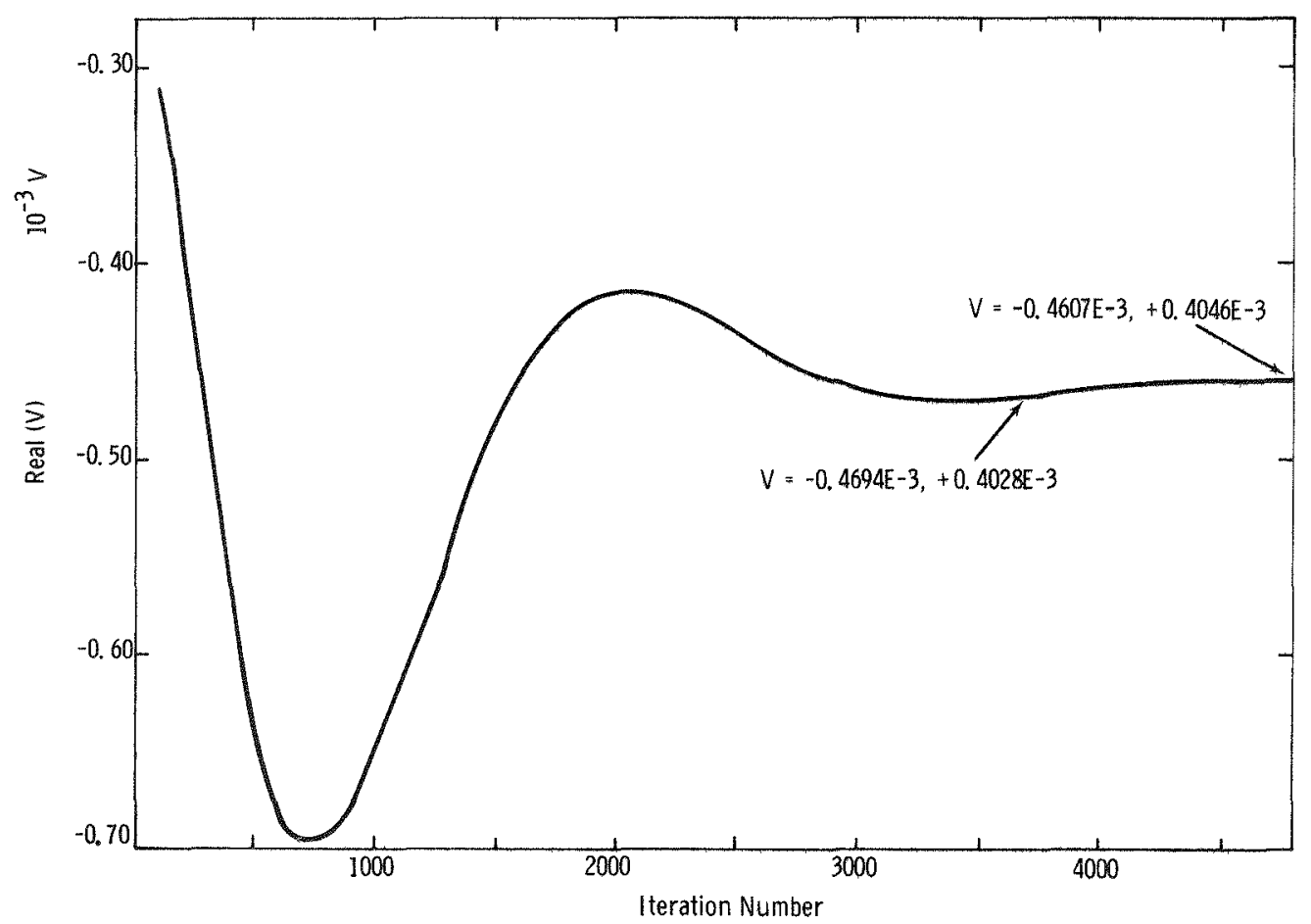

Figure D-4. Real Part of the Complex Voltage in a Receiver Coil at $\mathrm{L}=0.924 \mathrm{~m}$ for Test Problem $\mathrm{G}^{\prime}$ After Mesh Expansion (receiver coil radius is $0.024 \mathrm{~m}$ ) 


\section{Use of FIDELE (FInite Difference Solution for Magnetic Dipole Excitation of a Horizontally and Vertically Layered Earth) Computer Code}

A typical control stream for FIDELE is listed below. As illustrated in Appendix F, the control stream and the code are available as public files on the NOS system at SNLA. The file names are CFIDEL and FIDELE, respectively, and are filed under user number CNVITTI.

JOB CARD,MC7,T3000,EC400. NAME AND BOX ACCOUNT CARD

FILE,TAPE11, RT $=W$.

Compile program; get listing so that all parameters recorded.

FTN, R $=3$.

ATTACH,FXMATH,FXMATH.

LIBRARY,FXMATH.

If problem is a continuation from an earlier run, previous result must be input.

ATTACH,TAPE11,DIPMK-PROB2.

$\mathrm{LGO}, \mathrm{PL}=200000$.

Make new copy of result for cataloging.

FILE,TAPE1, RT $=W$.

REWIND, TAPE12.

COPYBE, TAPE12,TAPE1,1.

Purge old file; save new result for continuation or plotting.

PURGE,A1,DIPMK-PROB2,CN = ** . CATALOG,TAPE1,DIPMK-PROB2, CN $=* * *$, $R P=30$.

Make fiche copy of output for reference

REWIND,OUTPUT.

COPYCF,OUTPUT, FISH.

FICHE,FISH. PROBLEM

IDENTIFICATION

\section{8-9 END OF FILE} COMMENT

Insert FIDELE data; one blank card or comment suffices.
$6-7-8-9$
END OF INFORMATION

The code uses two files, Tape 11 and Tape 12. Tape 11 is read as input when IPL $=2$ or 3 , giving initial values for problem parameters including the $S$ matrix sought. At job completion, Tape 12 is used to store the current values of all problem parameters. Tape 12 is used for subsequent plotting of results (by means of DIPPL) or for problem restart with IPL $=2$ or 3 .

In application the user will want to add name and box to the job card and alter the account card. The permanent file name attached, purged, and cataloged for future use (DIPMK-PROB2) may also be altered. The XEDIT processor on NOS is convenient for such changes.

Use of FIDELE has been illustrated in several sections during discussion of the test problems. Test Problem $C$ results are preceded by a list of specific variables that are likely to change for a new problem. For example, the number of iterations (NITER) may be altered. The T3000 designation on the job card (3000-s CPU time) is appropriate for NITER $=1200$. Through the use of XEDIT the parameter is easily changed by searching for the variable in the FIDELE code.

\section{$\mathrm{L} / \mathrm{NITER}=1$}

When "NITER $=$ " is found, the alteration is quickly completed. Each of the listed variables may be checked in this manner.

Changes in the electrical parameters are controlled by Subroutine EP. Several possibilities are discussed in the sections for Test Problem $\mathrm{C}$ and for Test Problem F.

Slight alteration in code structure could allow input of these quantities by a few data cards. However, there is great generality in the types and numbers of layers that might be encountered geologically. It would be difficult to build such generality into the 
code, especially before experience in practical applications. In addition, insertion by data cards requires format and order descriptions. Frequency of use may later justify attempts to simplify problem input.

After the CFIDEL and FIDELE files are altered appropriately, the job is transferred to the CDC-7600 computer at SNLA by the command
$\mathrm{SENDJOB}(\mathrm{CC}=\mathrm{CFIDEL}, \mathrm{P}=\mathrm{FIDELE}, \mathrm{CS}=\mathrm{RO})$.

The RO specifies output to be sent to the computer room for Building 806 at SNLA. Other choices are described in the NOS News Notes generated by the SNLA computing personnel. 


\section{APPENDIX F}

\section{Use of the DIPPL Computing Code for Plotting Results of FIDELE}

DIPPL results have been presented earlier--for example, Figures 16 through 20 dealing with Test Problem $\mathrm{C}$. In addition to furnishing results in a form convenient for interpolating to nearby receiver-coil radii or to different vertical distances ( $L$ values) from the source coil in Figures 18 through 20, the results can also alert the user to input errors as in Figure 7. In addition to the graphical results already discussed, a plot of $r$ vs $t$ is also generated to illustrate the nonlinearity introduced by choice of gamma.

Input to DIPPL is furnished by a Tape 11 file generated in the FIDELE computer code. A typical job stream is illustrated below.

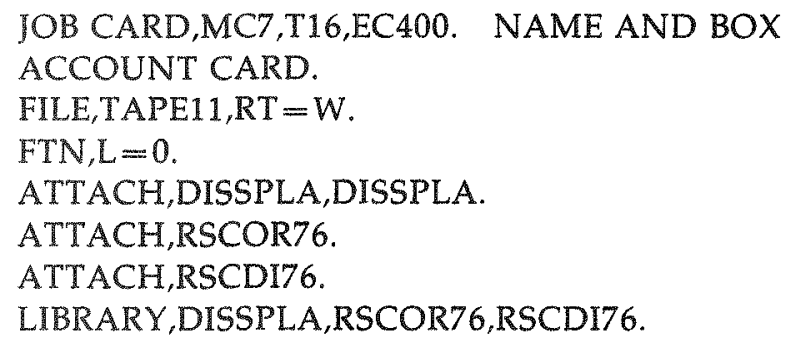

Get data from FIDELE code, cataloged on system at SNLA.

ATTACH,TAPE11,DIPMK-PROB2.

$\mathrm{LGO}, \mathrm{PL}=200000$.

Put results into file for processing by computing center.

ATTACH,POP,SCORPOP-7600,CY $=1$.

POP,TAPE77,TEMP,FICHE. DIPPL PROBLEM LABEL

REWIND,TEMP.

COPY,TEMP,BEMP.

LIBRARY, COMLIB.

Make hardcopy plots for rapid checking.

ATTACH, RHCNBP.

BEGIN,RHC,RHCNBP,BEMP.

Make microfiche copy of plots for storing or for generating better quality graphs.

COMQ,TEMP,DIC,FICHE, REV.

\section{7-8-9 END OF FILE}

DIPPL DATA - a blank card will suffice

6-7-8-9 END OF INFORMATION

The DIPPL code and a control stream reside as public files on the NOS system at SNLA. Use is illustrated below.

Get the job stream

$\mathrm{G}, \mathrm{CDIPPL} / \mathrm{UN}=$ CNVITTI,

XEDIT,CDIPPL.

Get the DIPPL code

G,DIPPL/UN $=$ CNVITTI

Send the job to the 7600 computer for processing, with return to station $R O$.

SENDJOB $(C C=C D I P P L, P=D I P P L, C S=R O)$

Need for alterations in DIPPL are not anticipated unless the form of Tape 11 is altered in FIDELE. Change of the S-matrix dimensions (NZ,NR) from $(201,201)$ will require corresponding dimension changes in DIPPL.

Instead of permanently keeping the public files CDIPPL and DIPPL (and also CFIDEL, FIDELE) as indirect access files, we will soon transfer them to public TFILE tape number 011892. After the transfer, each file may be recovered by the NOS command

TFILE,R,011892,NAME.

This command takes the file NAME (CDIPPL or DIPPL) from the public tape and stores it in the user's disk area as an indirect access permanent file. 


\section{APPENDIX G}

\section{Computer Time Requirements}

The timing and cost data are tabulated here for Test Problem F. These data are consistent with the other test problems. Three runs were required to complete the problem. Timing data are summarized below for the CDC 7600 runs needed for Test Problem $F$.

$\begin{array}{cccc}\text { Run } & \text { Iterations } & \text { Time(s) } & \text { Cost (\$) } \\ 1 & 300 & 746 & 90 \\ 2 & 2400 & 5865 & 682 \\ 3 & 1000 & 2448 & 287\end{array}$

The time required is approximately constant at $2.5 \mathrm{~s}$ per iteration. The cost varies from $28 \mathbb{4}$ to $30 \mathbb{4}$ per iteration, with the longer computer runs at the lower rate. A typical problem costs about $\$ 1000$ to solve at current SNLA computer costs.

The plotting program, DIPPL, requires less than 4 $s$ to run. Compiling the program, generating hardcopy and microfiche plots, and other input-output charges increases the time requirement to $14 \mathrm{~s}$.

Alteration of NR and NZ from their value 201 in FIDELE will alter these results and will also force changes in the plotting code DIPPL. 


\section{DISTRIBUTION:}

Amoco Production Co.

R 1, Box 467

Bixby, OK

Attn: R. Murphy

Amoco Production Co. (4)

P.O. Box 591

Tulsa, OK 74102

Attn: A. H. Jageler

M. B. Smith

R. W. Veatch

R. Wiley

Arco Oil \& Gas Co. (2)

P.O. Box 2819

Dallas, $\mathrm{T} X 75221$

Attn: F. Y. Michael

E. S. Pasternack

Canadian Hunter Expl 7004354 th Ave SW

Calgary, Alta., Canada

Attn: R. Hietala

CER, Inc. (2)

P.O. Box 15090

Las Vegas, NV 89109

Attn: G. C. Kukal

R. L. Mann

Chevron Oilfield Research Co. (5)

P.O. Box 446

La Habra, CA 90631

Attn: R. J. S. Brown

R. E. Davis

J. Gunter

C. H. Neuman

A. Timur

Cities Service Co.

P.O. Box 3908

Tulsa, OK 74102

Attn: Tian-I Shentwu

Columbia Gas System

1600 Dublin Rd

Columbus, OH 43215

Attn: R. Forrest
Dresser Atlas (3)

P.O. Box 1407

Houston, TX 77096

Attn: R. B. Culver

D. Sethi

A. H. Youmans

Exxon Production Research Co. (4)

P.O. Box 2189

Houston, TX 77001

Attn: C. Dutcher

J. Gidley

A. O. Gist

T. W. Muecke

Gas Research Institute

10 West 35th Street

Chicago, IL 60616

Attn: J. C. Sharer

Gearhart-Owen Industries, Inc.

Briarcliff 840

Spicewood, TX 78669

Attn: J. Moran

Gearhart-Owen Industries, Inc.

P.O. Box 1936

Fort Worth, TX 76101

Attn: M. Gearhart

Gulf Research and Development Co. (2)

P.O. Box 36506

Houston, TX 77036

Attn: E. L. Bigelow

J. M. Price

Gulf Research and Development Co.

P.O. Drawer 2038

Pittsburgh, PA 15230

Attn: R. P. Trump

Institute of Gas Technology (4)

3424 S. State

Chicago, IL 60616

Attn: P. O. Randolph

R. Rose

W. Rose

K. Samptah 


\section{DISTRIBUTION (cont):}

Lawrence Livermore National Laboratory

P.O. Box 808

Livermore, CA 94550

Atm: D. Towse

Los Alamos National Laboratory (3)

P.O. Box 1663

Los Alamos, NM 87545

Attn: J. N. Albright, MS 977

J. Brown, MS 329

J. Jackson, MS 329

Mobil Research and Development Co. (2)

P.O. Box 900

Dallas, TX 75221

Attn: W. F. Baldwin

J. L. Fitch

NL Petroleum Services/

NL Industries, Inc.

P.O. Box 1473

Houston, TX 77001

Attn: R. A. Meador

New Mexico Institute of Mining and Technology

Petroleum Recovery Research Center

Socorro, NM 87801

Attn: N. R. Morrow

Phillips Petroleum (2)

Bartlesville, OK 74004

Attn: W. Souder, 290 FPB

K. Stengel, 713 PRC

Schlumberger

4669 Southwest Freeway

Houston, TX 77027

Attn: Y. Boutemy

Schlumberger-Doll Research (2)

P.O. Box 307

Ridgefield, CT 06877

Attn: P. Day

P. N. Sen

Schlumber Well Services

P.O. Box 2175

Houston, TX 77001

Attn: R. N. Rau
Shell Development Co. (2)

P.O. Box 481

Houston, TX 77001

Attn: M. R. Williams

R. Saucier

Sohio Petroleum Company

50 Penn Place, Suite 1100

Oklahoma City, OK 73118

Attn: L. F. Elkin

Sohio Petroleum Company

100 Pine St, 20th Floor

San Francisco, CA 94111

Attn: L. F. Eklin

Stanford University

Department of Geophysics

Stanford, CA 94305

Attn: J. Walls

Terra Tek

420 Wakara Way

Salt Lake City, UT 84108

Attn: A. H. Jones

Texas A\&M University

College Station, TX 77843

Attn: J. Osoba

US Department of Energy (7)

Bartlesville Energy Technology Center

P.O. Box 1398

Bartlesville, OK 74003

Attn: H. B. Carroll

A. B. Crawley

J. B. Jennings

M. Lowe

C. Raible

L. Volk

D. C. Ward

US Department of Energy (3)

Office of the Deputy Assistant

Secretary for Oil \& Gas Technology

MS D-107

Washington, DC 20545

Attn: M. R. Adams

J. B. Smith

P. R. Wieber 
DISTRIBUTION (cont):

US Department of Energy

Nevada Operations Office

P.O. Box 14100

Las Vegas, NV 89109

Attn: C. H. Atkinson

USGS (2)

Denver Federal Center

P.O. Box 25046

Denver, CO 80225

Attn: G. R. Olhoeft

J. K. Pitman, MS 939

University of Texas (3)

Department of Electrical Engineering

Austin, TX 76019

Attn: F. X. Bostick

W. C. Dusterhoeft

H. W. Smith

University of Houston

Department of Electrical Engineering

Houston, TX 77004

Attn: L. C. Shen

Welex (3)

P.O. Box 42800

Houston, TX 77042

Attn: D. M. Arnold

J. W. Minear

W. J. Sloughter

1000

G. A. Fowler

1100

C. D. Broyles

1110 J. D. Plimpton

Attn: C. R. Mehl, 1112

C. W. Smith, 1112
1120

4231

4231

4400

4500

4510

4700

4710

4740

4742

4746

4748

4750

4753

4754

4754

4754

4754

4754

4754

4755

5500

5800

8214

3141

3151

DOE/TIC (25)

T. L. Pace

H. E. Viney

Attn: B. G. Edwards, 1131

W. C. Vollendorf, 1133

T. J. Grissom

D. F. Cowgill

G. Yonas

J. E. Powell

T. P. Wright

C. N. Vittitoe (3)

A. W. Snyder

E. H. Beckner

W. D. Weart

J. H. Scott

G. E. Brandvold

R. K. Traeger

A. F. Veneruso

B. Granoff

B. E. Bader

V. L. Dugan

D. A. Northrop (2)

C. L. Schuster

T. L. Dobecki

L. E. Duda

P. C. Lysne (5)

L. K. Warne

J. S. Yu

R. L. Fox

O. E. Jones

Attn: W. Herrmann, 5530

R. S. Claassen

M. A. Pound

L. J. Erickson (5)

W. L. Garner (3)

For: DOE/TIC (Unlimited Release)

(C. H. Dalin, 3154-3) 